University of San Diego

Digital USD

1994-06-01

\title{
The Lived Experiences of Nurses' Interactions with Ethnically Diverse Clients: A Phenomenological Perspective
}

Colette R. York DNSc, MSN, RN

University of San Diego

Follow this and additional works at: https://digital.sandiego.edu/dissertations

Part of the Nursing Commons

\section{Digital USD Citation}

York, Colette R. DNSc, MSN, RN, "The Lived Experiences of Nurses' Interactions with Ethnically Diverse Clients: A Phenomenological Perspective" (1994). Dissertations. 244.

https://digital.sandiego.edu/dissertations/244

This Dissertation: Open Access is brought to you for free and open access by the Theses and Dissertations at Digital USD. It has been accepted for inclusion in Dissertations by an authorized administrator of Digital USD. For more information, please contact digital@sandiego.edu. 
UNIVERSITY OF SAN DIEGO

Philip Y. Hahn School of Nursing

DOCTOR OE NURSING SCIENCE

THE LIVED EXPERIENCES OF NURSES' INTERACTIONS

WITH ETHNICALIY DIVERSE CLIENTS:

A PHENOMENOLOGICAL PERSPECTIVE

by

Colette R. York, R.N., M.S.N.

A dissertation presented to the

FACULTY OF THE PHILIP Y. HAHN SCHOOI OF NURSING

UNIVERSITY OF SAN DIEGO

In partial fulfillment of the requirements for the degree DOCTOR OF NURSING SCIENCE

June 1994 


\begin{abstract}
The Lived Experiences of Nurses' Interactions with Ethnically Diverse Clients: A Phenomenological Perspective
\end{abstract}

This study explored the phenomenon of nurses' lived 4experiences while interacting with clients who were ethnically dissimilar to themselves in a variety of nursing care settings including acute care, ambulatory care and public health. This study is timely, especially in the county in which the study was conducted because of the ongoing influx of legal and illegal immigrants from diverse foreign geographic locales.

Van Kaam's method for conducting phenomenological studies was employed for data gathering, categorizing and analyzing. Categories were stated in terms of perceptions and feelings. The most frequently stated perceptual moments included perceiving client ethnicities based on physical characteristics, language fluency, beliefs and practices, and presumptive clues such as address or surname. Nurses also drew conclusions about their clients' ethnicities based on past, personal experiences.

At the emotional level, there emerged a blend of both pleasant and difficult feelings. Nurses described experiencing feelings of heightened awareness, empathy and 
compassion toward their patients. There were also moments when, even in light of language difficulties, the nurses felt accepted and trusted by the careseekers. Others experienced feelings of triumph, satisfaction and reward. The pleasant emotions were at times tempered by feelings of inadequacy, frustration, anger and pressured by time constraints. For some participants, the experience of "being discriminated against" by clients was described. As a result of the information emerging from this study, a synthetic description of the phenomenon was composed.

The study included tape-recorded interviews with fifteen nurses who were themselves members of diverse ethnicities. An interview guide was prepared and utilized to assist the volunteers in recalling the most memorable interactions in which they were not members of the patient's ethnicity. These participants were chosen through a snowball sampling approach. All measures were taken to insure the confidentiality of the nurses and their clients and to maintain trustworthiness, dependability, confirmability, subjectivity, accuracy and truth. Although phenomenological findings are not generalized beyond the study participants, insight can be gained into the meaningfulness of nurses' experiences which provide additional research questions and implications for nursing practice, education and research. 
This dissertation is dedicated to my husband and best friend, Ron, my living children, Jennifer, Keith, Suzanne and to the memory of our son, Robert, who through his death, taught me the fragile nature of the human spirit. He also inspired me to reach beyond my own grief and begin my doctoral education, at first to fill my empty days.

I also dedicate this dissertation to my dearest friends the Miller family, the Blais family, to Carol Frank and to my close women friends for their love, support and ongoing friendship even though they could not fathom my reasoning for returning to school.

I want to extend my heartfelt gratitude to all those dedicated nurses who became the participants for this study. Their beautiful stories make up the essence of this dissertation and reinforced the need to retell their stories.

I also wish to dedicate this dissertation to the members of my committee and to Dr. Merry Armstrong who extended themselves in offering direction, suggestions and support.

To Vickie, who became my typist and transcriptionist, I extend much gratitude for helping me to meet deadlines and keeping me organized. 
TABLE OF CONTENTS

Page

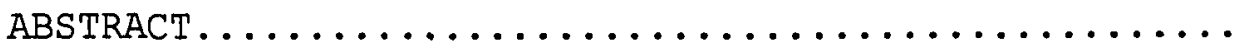

DEDICATION............................. ii

TABLE OF CONTENTS...................... ii

LIST OF TABLES ....................... viii

LIST OF APPENDICES..................... ix

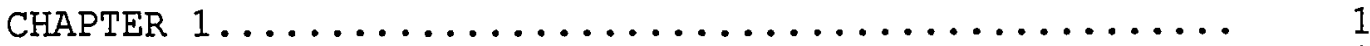

INTRODUCTION.......................... 1

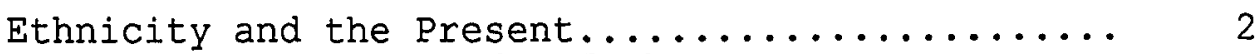

Rationale for the Study of the
Nurse/Client Multiethnic Interactions........

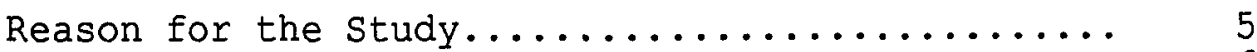

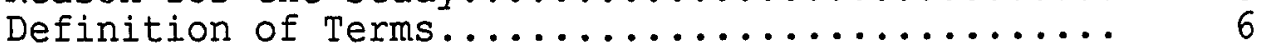

Researcher's Assumptions.................. 7

Historical Significance Influencing the Study.... 8

Summary......................... 11

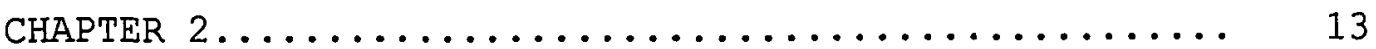

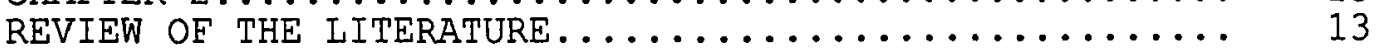

Historical Development of

Phenomenology................... 14

The Goal of Phenomenology as a Research

Design...................... 18

Phenomenology as a Nursing Research

Method...................... 19

Beliefs, Attitudes and Experiences as

Influences in Human Interactions....... 24

Labeling or Stereotyping as a Process...... 28

Acquisition of Stereotypic Views.......... 31

The Question of Ingroup/Outgroup

Membership............................. 34

Living Proximity Influencing Social

Interactions................. 36

iii

Reproduced with permission of the copyright owner. Further reproduction prohibited without permission. 
Social Forces as Contributing to Labeling... 37

Significance of Personal, Relational and

Social Contacts................. 39

Research into the Relevance of Stereotyping...... 40

Consequences of Mental Images............ 47

Interethnic Relationships between Nurses and Clients and Unifying Factors...... 49

Interactional Dynamics.................. 52

Language Differences and Paralanguage....... 53

Dress and Body Adornments as Cues........... 54

Awareness of Self and Sensory Reactions..... 55

Emergence of the Therapeutic Model........ 57

Summary........................ 58

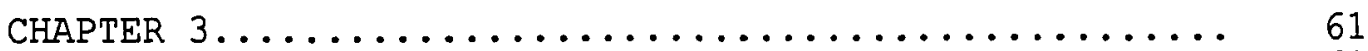

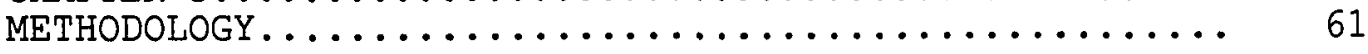

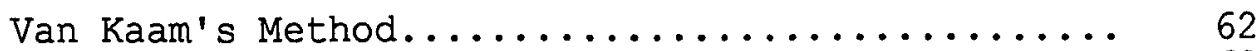

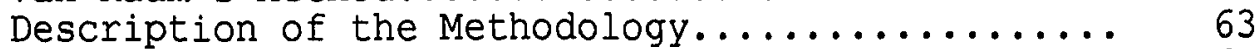

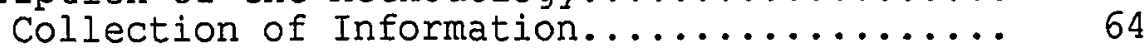

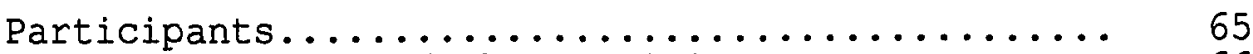

A Composite of the Participants........... 66

Method for Selecting the Participants....... 67

Interview Process.................. 69

Major Questions and Probes............. 70

Listing and Preliminary Grouping of

Perceptions and Feelings........... 72

Reduction and Elimination

of Data and the Usefulness of Experts.. 73

Hypothetical Identification............. 75

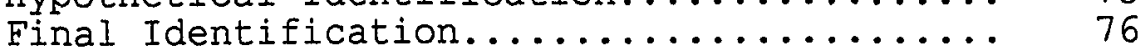

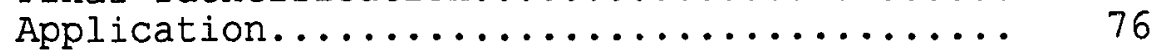

Data Analysis: Process in the Identification of

Constituents....................... 77

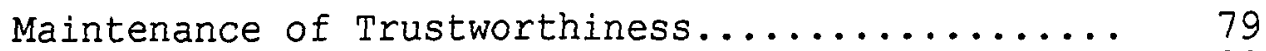

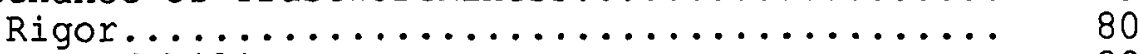

Dependability...................... 80

iv 
TABLE OF CONTENTS (Continued)

Confirmability and Subjectivity......... 81

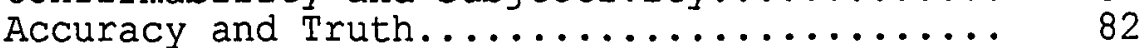

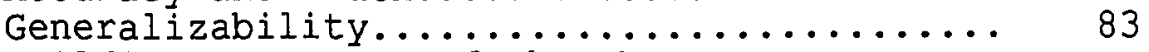

Building Awareness of the Phenomenon

through Language............... 84

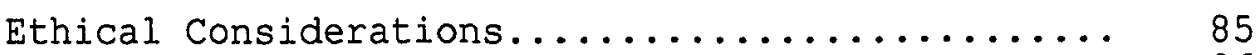

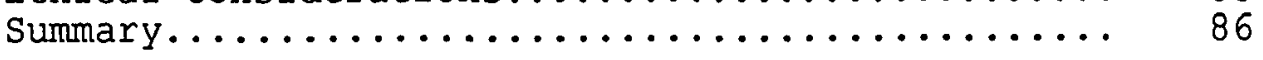

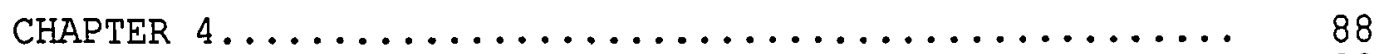

PRESENTATION OF THE FINAL CONSTITUENTS............ 88

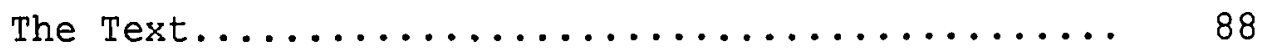

Level I Perceptions (Object Directed)

Becoming Aware of Client's Ethnicity:..... 89

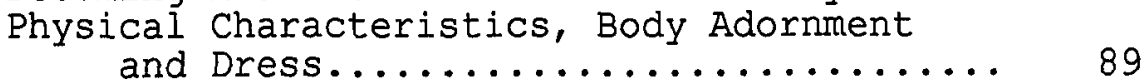

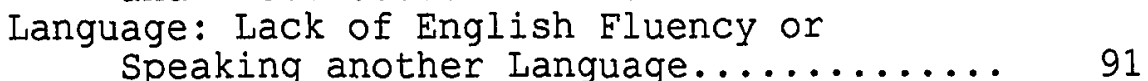

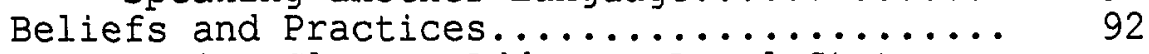

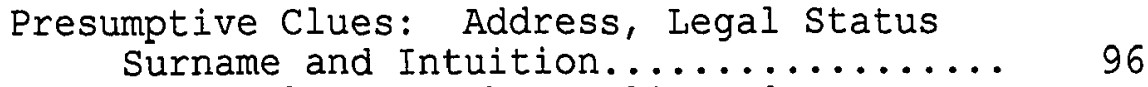

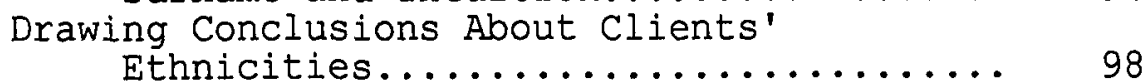

Role of past experiences............ 99

Making Assumptions About Clients......... 108

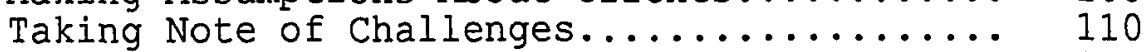

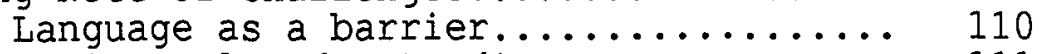

Reciprocal understanding........... 111

Level II: Feelings (Subject Directed)......... 118

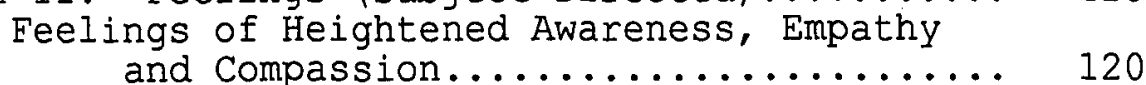

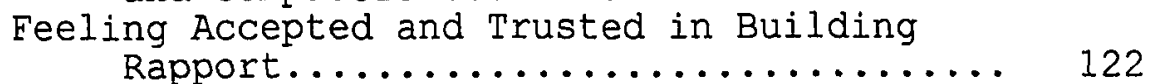

Feeling A Sense of Triumph, Rewarded and

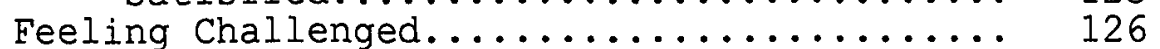

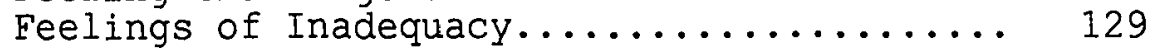

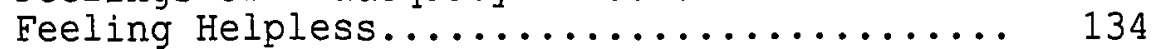

Feeling Frustrated and Angry.............. 134

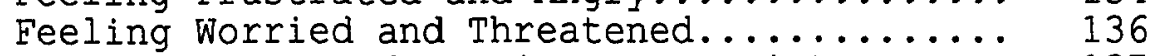

Feeling Pressured By Time Constraints....... 137

Feeling Resentment.................. 138

\section{V}


TABLE OF CONTENTS (Continued)

Feeling Discriminated Against By Clients.... 139

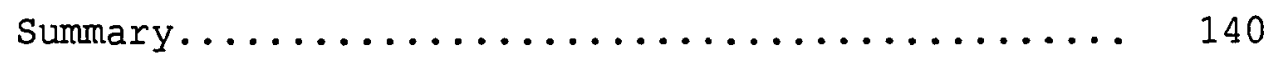

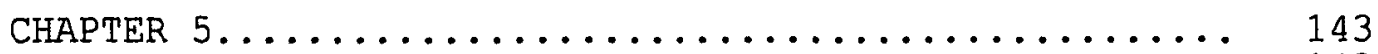

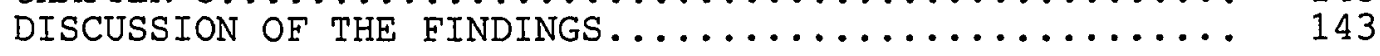

Synthetic Description of The Phenomenon........ 143

Enlightened Awareness..................... 144

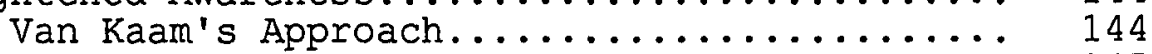

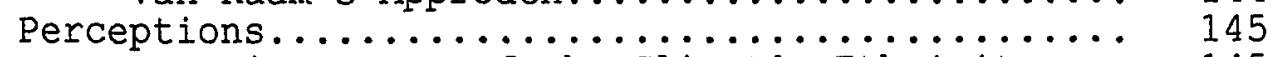

Becoming Aware of the Client's Ethnicity.... 145

Drawing Conclusions About Clients.......... 147

Making Assumptions About Clients.......... 148

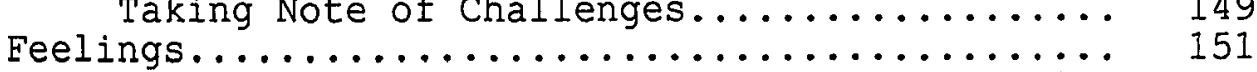

Feelings of Heightened Awareness, Compassion

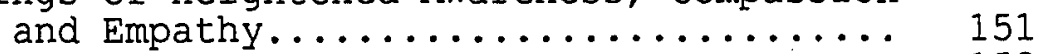

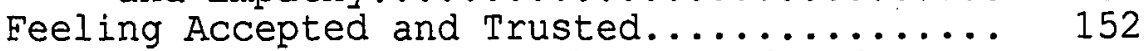

Feeling A Sense of Triumph, Reward and

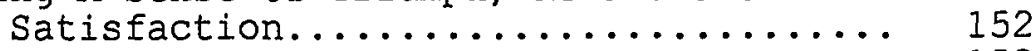

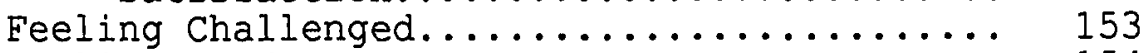

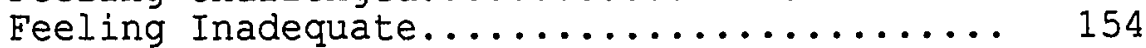

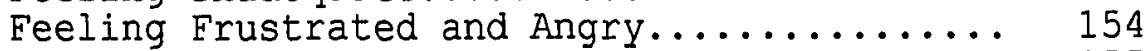

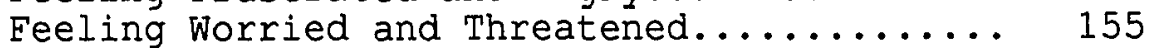

Feeling Pressured By Time Constraints....... 156

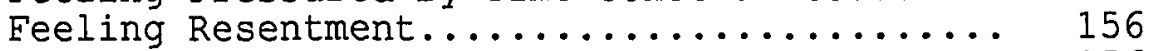

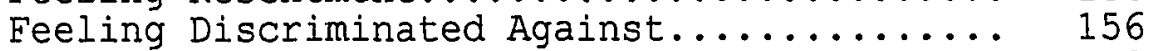

Role of the Researcher's Observations........... 158

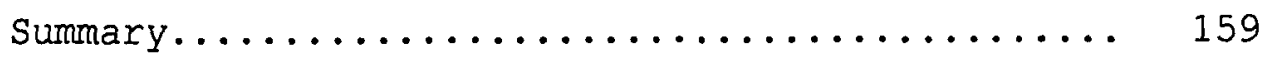

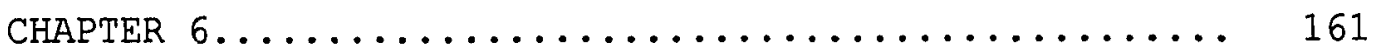

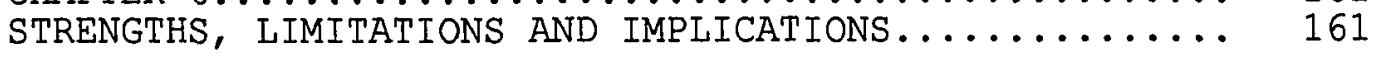

strengths............................. 161

Limitations........................ 164

Limitations of Phenomenological Approach.... 165

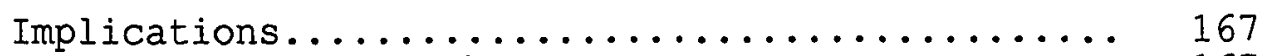

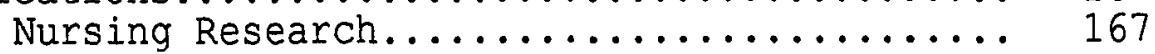

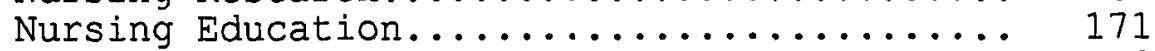

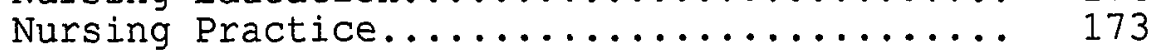

vi 
TABLE OF CONTENTS (Continued)

REFERENCES.......................... 176

vii

Reproduced with permission of the copyright owner. Further reproduction prohibited without permission. 


\section{LIST OF TABLES}

TABLE NUMBER TITLE

PAGE NUMBER

1

Demographic Attributes of the

Participants

Professional Preparation and Areas

of Specialization

3

Final Constituents of the Phenomenon

142

viii 
APPENDIX A

UNIVERSITY OF SAN DIEGO COMMITTEE ON THE

PROTECTION OF HUMAN SUBJECTS................ 197

APPENDIX B

UNIVERSITY OF SAN DIEGO CONSENT TO ACT AS A

RESEARCH PARTICIPANT................... 202

APPENDIX C

DEMOGRAPHIC QUESTIONNAIRE............... 204

APPENDIX D

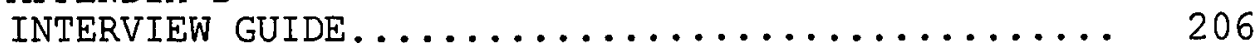

APPENDIX E

LETTER TO THE PANEL OF EXPERTS............. 208

APPENDIX $\mathrm{F}$

THE PRELIMINARY CONSTITUENTS OF THE PHENOMENON... 210

APPENDIX G

STATEMENTS THAT IDENTIFY CONSTITUENTS OF THE

PHENOMENON 
Chapter one

INTRODUCTION

Despite their role as professionals, nurses are first, citizens of the world. As such, they may develop assumptions about members of particular ethnic groups, affix positive or negative labels to people and change the course of the patient/nurse dyadic relationship. Existing in the world is not enough to build knowledge about it. One must draw information from others to truly understand the meaning of life through personal experiences and experiences of others. Buber (1958) identified this premise as the "I and Thou" philosophy.

Some nursing authors have mentioned that ethnicity, as a phenomenon, was worth exploring in nursing because dissimilarities between the caregiver and the care recipient, can result in either inappropriate treatment or care modalities (Butrin, 1992). Harwood (1981), a physician, stated that from the patient's point of view, "...healthcare has come to focus more and more on the treatment of abnormalities in the structure and function of body organs and systems, with a resulting neglect of concern with patients' experiences of these abnormalities" (p. 1). 
The profession has spent decades building the skills and professional levels of its members. Benner (1984) explored the process by which nurses build expertise through experience. However, what has not been clearly defined is the role of nurses' feelings regarding their individual experiences. This can become evident in allowing nurses to express their own stories. Exploring the lived experiences' of nurses interacting with ethnically diverse patients may assist in identifying the true essences of these experiences and the associated feelings and perceptions. The ability to recollect these interactions as they were lived by the nurses may enhance learning. The retelling of personal experiences is most effective as outlined in Dale's "Cone of Experience" (Wiman, Mlerhenry, 1971). People generally retain $70 \%$ of what they write or verbalize compared to $20 \%$ of what they hear, read or visualize. One can then hypothesize that if nurses are given the opportunity to relate their stories, their experiences may be mentally retained and eventually assist these caregivers in providing ethnically sensitive care while living through future and similar experiences in their individual practice settings.

\section{Ethnicity and the Present}

During the last twenty years, ten million immigrants have sought refuge in the United States. An estimated 700,000 immigrants legally enter the United States each 
year. Of these immigrants, three out of every four were born in countries other than Europe. California is considered to be the fastest growing state and has absorbed $35 \%$ of all Hispanics and 39\% of all Asian emigres. In 1990, only 57\% of Californians claimed European ancestry. A recent CNN/USA Today/Gallup poll revealed the beliefs that Americans want the federal government to restrict immigration. Of those included in this survey, $65 \%$ wanted immigration curtailed and specifically identified the ethnic groups they believed to have contributed to either the problems or the benefits in this country. ("Reduced Immigration", 1993; San Diego Union, 3/11/91).

Because San Diego County will continue to be a border city, immigrants from not only Mexico but from Latin American and South American countries will continue to cross the border, both legally and illegally, in an attempt to improve their life situations. Eventually, immigrants will also enter the healthcare system in one or several settings and will interact with ethnically diverse nurses who might become their primary caregivers. The results of the nurse/patient encounters have been documented from the patients' perspective, however a void exists in uncovering the stories of nurses immersed in these situations (Butrin, 1991; Kramer, 1992; Los Angeles Immunization Demonstration Project, 1991; Spector, 1991). 
When nurses do not understand the beliefs, behaviors and practices of individuals who are ethnically different than themselves, the phenomena of knowing, challenging, misunderstanding, labeling, rejecting or judging can occur and are worthy of investigation. To date, many nursing authors have explored and explained the patient's experience but limited research exists that investigates the nurses' lived experiences with a multitude of ethnic clients (French \& Schwartz, 1990; Leininger, 1988; Spector, 1992).

Rationale for the Study of the Nurse/Client Multiethnic Interactions

Researchers who explored labeling, stereotyping and prejudicial thinking have concluded that the less information one has concerning another individual, the greater the danger may be for misunderstanding and judging especially as it relates to ethnic and cultural issues (Ashmore \& Del Boca, 1981; Bierhoff, 1989; Bullough \& Bullough, 1982; Fishbein, 1967; Fiske \& Taylor, 1983; Hamilton, 1981; Harwood, 1981; Miller, 1982; Stephan \& Rosenfield, 1982; Taylor, 1981; Tedeschi, 1984). When health is compromised and the patient's needs are dependent on specific actions by nurses, misunderstanding can potentiate control issues and alter both healthcare delivery and nursing intervention. In addition, the client may negatively perceive the care experience and become lost to 
the system. In essence, this experience may affect the self esteem of the care recipient. A lack of knowledge concerning the patient may promote feelings of inadequacy among nurses who may also perceive a lack of control regarding the patients' health outcomes. Judgements about the patient and the entire group to which the patient claims membership may result. Consequently, a sense of failure ensues in this dyadic relationship (Branch, 1978; Leininger, 1978).

\section{Reason for the study}

Nurse researchers (Henderson \& Primeaux, 1981; Leininger, 1988; Lightfoot-Klein \& Shaw, 1990; Orque, Bloch \& Monrroy, 1983; Spector, 1985) have studied patients from various ethnic and cultural groups. However, there remains a paucity of research that explores the nurses' feelings in the context of ethnically dissimilar patient/nurse dyads. Recognizing that both nurses and their clients are ethnically diverse, nurses will eventually care for clients who are not of their particular ethnicity or culture. In these encounters, there will be moments of pleasure, challenge, frustration and a lack of awareness regarding the client's persona. The question to be answered in this research is, "What are the lived experiences of nurses who interact with clients who are ethnically different than themselves?". Nurses will be asked to recall the perceptual 
and emotional moments of the experiences when the patient's ethnicity was dissimilar to their own. These descriptions will then be labelled and categorized according to similarities and the frequency with which they are mentioned. Common descriptions will emerge to create an accurate vision of the phenomenon as lived by these nurses.

\section{Definition of Terms}

To more clearly comprehend the ethnically diverse interaction that will be explored in this study, a definition of terms is warranted. Neufeldt and Guralnik (1991) defined ethnicity as a group of people who have a common heritage that provides its members with a common language, historical perspective and customs. Orque, Bloch and Monrroy (1983) viewed ethnicity as an affiliation of persons sharing a unique background that influences their beliefs, goals and language. The word, culture, is frequently used interchangeably with the word ethnicity. It has been defined as the sum total of a person's way of life, including physical characteristics, language, dress, foods, beliefs, health practices, behaviors, religion, nurturing and kinship systems. Spector (1985) summarized culture as a person's life time "luggage" (p. 50). For some writers, ethnicity is a more specific social unit contained within a culture and predominantly refers to a person's origins and membership in a social unit. A "collectivity of 
people" bound by shared histories, beliefs and mores is the label employed by Harwood to define ethnicity (1981, p.3). In contrast, race refers to one's biological characteristics such as, skin color, eyes, hair, body shape, blood types, and genetic patterns (Lawless, 1979; Leininger, 1978; Neufeldt, 1991; Spector, 1985).

\section{Researcher's Assumptions}

The assumptions of this researcher are:

1. Nurses not familiar with patient's ethnic beliefs and practices will be able to describe their perceptions as well as their feelings while interacting with these patients.

2. Nurses will describe beliefs and attitudes they hold about certain ethnic groups.

3. Nurses will be able to describe the labels or stereotypes they have affixed to particular patients because of the latter's ethnicity.

4. Nurses will be able to recall specific moments or encounters in which they experienced feelings of awareness, empathy, challenge, inadequacy or frustration.

5. Nurses will also be able to describe how these experiences have presented challenges for them in communicating, understanding or being understood 
by client representatives of the ethnic groups with whom they have interacted.

Historical Significance Influencing the Study According to Spector (1985), the United States experienced a major migration movement between 1820 and 1975. Within the first ninety years of this period, the new arrivals originated predominately from England, France, Germany and Belgium, followed by citizens from Italy, Ireland, Hungary, Austria, Russia and Canada. Kalisch (1986) added that others immigrated from Yugoslavia, Spain and Portugal. During this historical period, the United States became a multiethnic society with representation from most European countries. Following this epoch, approximately one million emigres from Southeast Asian countries resettled in states such as Washington, Oregon and California (Gilman, Justice, Saepharn \& Charles, 1992). Another mass exodus of Afghans followed the Soviet invasion of Afghanistan in 1979. Of these displaced people, ten to thirty five thousand settled in California alone (Lipson \& Omidian, 1992). All arrived with their specific illnesses and healthcare needs that already have or will eventually challenge nurses. Poverty, disease and life-threatening illnesses often resulted from overcrowding and poor sanitation (Kalisch, 1978). Compounding the health problems were such issues as language, ethnic and cultural issues. 
After the Spanish American War, concerns focused on the healthcare needs of a growing population of new immigrants. Through the efforts of leaders such as Lillian Wald and her establishment, called the Henry street settlement in New York City, nurses began to live among the new settlers, befriended them and ministered to their healthcare needs (Brainard, 1985; Coss, 1989; Kalisch, 1986; Marshall, 1972). Today, in San Diego County as in other states, different groups of immigrants continue to arrive in search of a more comfortable and safer life. For nurses, these new clients present unfamiliar health beliefs and practices in the nurse/client relationship. Although nurses have cared for culturally and ethnically diverse patients since before the turn of this century, there continues to be a dearth of research about the effects of these differences in the nurse and patient caring dyad. By exploring the lived experiences of nurses and by listening to their voices, data can be abstracted that may effect nursing education, research and practice.

Learning to identify, understand and confront the nursing profession's own informational and educational needs may help to heal both the frustrations of the nurses and of the clients who trustingly place themselves in the nurses' care. Leininger's (1978) statement was succinct when she acknowledged that cultural and ethnic awareness in nursing continues to be the "missing link" (p.17). As early as 
1980, the American Nurses' Association's Social Policy

Statement claimed that it is the society in which nursing practices that determines the profession's most pressing needs.

Within San Diego County, there is a critical demand to expand an informational framework that addresses the caring practices of an ever flourishing pluralistic patient population. Although more group-related information is becoming available to the profession via publications and research, the nurse continues to be placed in environments where education about patient ethnicities and cultures is frequently learned by trial and error. Without research that considers the nurses's voice in a variety of experiences, the profession will never be able to identify how it can support and optimize the patient/nurse interaction.

Although transferability of the findings in phenomenology is limited to the target population, information can be gleaned from this study that may create a significant basis for other investigations. The importance of considering many nursing-related problems from the perspective of nurses lived experiences may create a caring milieu in which nurses feel empowered to offer suggestions or systems that do or do not support their practices. Results might create a new awareness of nurses' experiences. These experiences can then be incorporated into nursing 
education to provide active learning opportunities rather than the role modeling approach, that is considered passive learning and is often implemented by educators (Kavanagh \& Kennedy, 1992; Meleis, 1991). Hopefully, the results of this study may also stimulate the development of strategies that support nurses through these multiethnic experiences.

\section{Summary}

In San Diego County, thousands of immigrants cross the United States border, both legally and illegally, in search of improved human conditions. Unfortunately, these immigrants also become ill and present for care in various healthcare settings where they may be confronted by nurses whose knowledge concerning their culture may be lacking. At the core of these experiences, is the quality of the interactions, the perceptions and the lasting impressions of the participants. Each interaction affects the relationship between both parties as well as subsequent ones.

Nurse researchers have explored the role of ethnicity from the patient's perspective. However, few studies have examined the experience from the nurses' vantage point. This study will explore or identify the voices of the participant nurses by utilizing a phenomenological research design. The primary study question is, "What is the lived experience of nurses who interact with patients who are ethnically diverse?". The hope is that the participants 
will be able to describe their personal feelings and perceptions while living out these experiences. By categorizing and analyzing these nurses' responses, the researcher will gather information that may become instrumental in either stimulating future research or changing the course of education and skill building in order to enhance ethnic awareness and sensitivity. In addition, the study may render opportunities to implement support measures for nurses who are faced with these experiential opportunities. 
Chapter Two

REVIEW OF THE LITERATURE

The patient/nurse dyad is a uniquely intimate one. Patients seek health and nursing care when their perception of health or illness is such that it requires intervention. For a time, there is a loss of control to someone who can help them return to a pre-intervention state. For many, the healthcare setting is unfamiliar, perhaps incongruent with ethnic or cultural health beliefs, practices, language or expressions. Simply stated, the experience renders the client temporarily in a state of powerlessness over health, personal and treatment issues (Fiske \& Taylor, 1983).

The other side of the equation is the caregiver, whose professional responsibilities dictate appropriate treatment. Nurses enter the dyad with personal, ethnic, cultural and professional characteristics that may or may not conflict with those of the careseeker. What are the outcomes of these interactions? What is gained, lost, misunderstood or appreciated? How does this pair become known to each other, or affected and changed? How is the experience labelled? Finally, how does it affect future, similar experiences? The goal of this research is to discover the answers through 
the voices of nurses.

The literature was reviewed in light of anthropology, education, nursing, philosophy, psychology and sociology in an attempt to understand the roots and outcomes of human interactions. An encounter of two people in a discrete relationship does not end without having affected both parties in ways that alter one's personal cognitive repertoire which may be recalled in future similar circumstances.

Historical Development of Phenomenology

A phenomenological research method was implemented for this study. An exploration of the philosophical underpinnings of this method seems appropriate to set up the foundation for the review of the literature. The thoughts of a few philosophers will be shared in order to provide some base for understanding phenomenology.

Plato is credited for introducing the word "Eidos" translated later by Husserl as meaning the essences of any experience (Van Manen, 1990, p. 177). Plato influenced phenomenologists with his doctrine of recollection as crucial in the theory of knowledge acquisition. For him, phenomena were manifestations of human sensory experiences and representations of the real world (Morse \& Henderson, 1973; Sahakian, 1968). Aristotle's point of view was that the universe was understood through the exploration of 
phenomenon that assisted in comprehending humans who are in a constant state of becoming.

Hegel's existential framework influenced phenomenology because of his belief that its method of inquiry allowed for an exploration of the human mind and its displayed activities. He concluded that the mind was a dynamic process constantly developing in a directional movement. Kant posited that only the person living through an event would have the knowledge about it and be able to describe this experience (Frost, 1962). In Brentano's opinion, human experience was the essence of philosophical discovery and he coined the term "intentionality" defined as the "...counterpart of an object" (Sahakian, 1968, p. 328). Phenomenology was viewed as a thorough dissection of everyday human experiences (Frost, 1962; Ricoeur, 1967; Van Manen, 1991).

One of the most influential philosophers in modern times was Husserl, who was considered the father of phenomenology. His premise was that uncovering common truths through reflection, as a return "to the things themselves", became basic tenets of his philosophy (Farber, 1968; Morse \& Henderson, 1973; Sahakian, 1968). Originating with Husserl was Spiegelberg's (1982) use of the term "bracketing" explained as a setting aside of one's own knowledge of an experience in order to explore the lived experiences of others in a relatively bias free manner. 
This can enable a return to the "things themselves" or to the basic essences of the experience (Scudder and Mickunas, 1985, p. 9).

of value, in phenomenology, is the idea of intersubjectivity, which is understood in terms of each individual's perception of the experienced phenomenon. Husserl explained that the aim of phenomenology was to describe the elements of a phenomenon, and return to the "final source in which all possible form of knowledge is functionally grounded" rather than to explore only the givens of an experience (Sinha, 1969, p. 2). The question of subjectivity then becomes a matter of some fundamental truth known only by those who experience a phenomenon (Sinha, 1969). In addition, subjectivity lies in the perception of truth relevant to a phenomenon that is experienced by an individual. The subjective nature of truth is considered as inherently personal. In the experiential world of lived events, there is logical meaning that can be explored and made evident (Sinha, 1969). In the development of phenomenologic inquiry in nursing, the philosophies of Marcel and Heidegger became very influential. Although both admitted that there are discrepancies between fact and personal interpretation, truth remains in a constant state of scrutiny by individuals who are living in the phenomenon (Morse, 1973). As other philosophers, like Tillich, introduced the element of 
transcendentalism into phenomenology, the interpretation of additional realities and their meaning became more diversified. This approach became accepted as more humanistic because it was accompanied by faith in whatever presented itself while searching for meaning (Frost, 1969; Hutchinson, 1975). For Marcel, the nature, as well as the meaning of a phenomenon, was essential. Understanding reality, from a phenomenological perspective, was likened to solving a mystery (Van Manen, 1990).

Heidegger's (1962) philosophical premises were based on much of Husserl's work. The idea of "being" or "Dasein" was as fundamental to Husserl's philosophical thought as were his views on the nature of time, of mankind and the world as fused not one existing without the other and that truth presented itself by unveiling this reality (Heidegger, 1962). In order to uncover reality, the reflection upon and recollection of past events and experiences are necessary. This allows for the understanding of meaning as a consequence of a person developing self awareness and understanding through experiential moments, thus transcending the material universe and becoming closer to a personal essence (Frost, 1962; Sahakian, 1968). Humans are capable of describing their own experiences through perceptual and emotional moments (Morse, 1973). People enter into a relationship with their life events in order to experience them fully (Sahakian, 1968). 
Spiegelberg (1982) posited that there was more to an object than was initially apparent to some individuals. Experiences can become the borders that encompass the object source. The research methodology developed by Spiegelberg has as a basic premise the researcher's preparation to not only hear but see the phenomenon as described by the participants. To him, all phenomena were essentially private and only known through a priori insight. Spiegelberg (1982) was not a proponent of hypothesizing about a phenomenon but believed that the experience would become self-evident through subjective data.

Clarification of humankind's participation in the world has been the goal of phenomenology since its inception. Description of attitudes and traits through this form of inquiry was geared toward envisioning the lived and human condition. Reality begins at the moment of perception and is perceived differently by each person. Phenomenology explains the primordial or primitive world form. Reality is not limited to what is absorbed through the senses but how the mind translates the data. One individual will never experience an event as another does (Merleau-Ponty, 1964).

\section{The Goal of Phenomenology as a Research Design}

The world is full of objects and social interactions. Phenomenology uncovers the phenomenon in question in terms of how it appears and is lived by others. According to 
Heidegger (1962), experience renders meaning to existence. Phenomenology converts the abstract into tangible meaning. This type of inquiry is defined as the description of human lived experience in an attempt to envision the individual experience rather than only to hear or listen to the account. The inquiry explores a lived event by the individuals who have been integrated in it. Personal reality is subject to both sensual observations and mental translations of events. As experiences unfold, humans maintain experiential information and utilize it in similar situations (Heidegger, 1962; Merleau-Ponty, 1964).

The goal of phenomenology is to understand meaning as perceived by those who have been involved in it (Heidegger, 1962; Merleau-Ponty, 1964; Oiler, 1982; Ricoeur, 1967; Van Kaam, 1966). For this study, the essences of nurses' lived experiences with ethnically diverse clients were uncovered as related to the phenomenon in question.

\section{Phenomenology as a Nursing Research Methodology}

Nurses have been described as keen observers of human interactions and therein lies the secret of discovery in this profession. In order to appreciate the role of phenomenology in this discovery, there is a need to review its influence in nursing theory development and research. As a profession, nursing has been in a constant state of becoming for decades. Phenomenology takes this process into 
account, whether the method is implemented to explain a singular experience or several experiences.

In nursing, theoretical perspectives are in need of constant development and refinement. Phenomenological inquiry and this profession seem to possess a common bond because both are inclusive of a humanistic view and the search for knowledge that considers personal experience holistically (Oiler, 1982; Omery, 1983; Watson, 1985). In addition, phenomenology not only affords nursing a research methodology but a philosophy in which the human being can be more thoroughly understood, not only as a unique individual but as one in the midst of a world of experience. This method answers the more humanistic questions that often remain unclearly revealed. Some quantitative research methods do not inquire about the essence of experience or about those events that tend to target the humanness of being, existing and experiencing. Developing some basic knowledge about the framework of phenomenology will enable nurses to appreciate their own history and the need for keeping the momentum going to gain insight into human experiences (Oiler, 1982; Omery, 1983; Watson, 1985).

Nursing theorists declared that frequently, there are no givens, no answers to the human questions; that the patient/nurse dyad is one of intersubjective transactions in which both client and nurse are unique and bring to the care setting all of their experiential world. The profession is 
humanistic in that it encompasses both the caregiver and the careseeker's needs for goal attainment. The latter is recognized as a capable being, responsible for making choices and the former is recognized as a potentiator of this goal in terms of health. A dialogic relation is created in which two unique beings interact in a constant state of becoming (Meleis, 1991; Parse, 1981; Paterson \& Zderad, 1976).

Benner (1984) observed that what is missing in nursing is the observational component of the nurses' learning experience and its development through practice. In her writings, Benner stated that nursing science demands expertise based on experiential events and descriptions of practice presented for interpretation. According to Benner (1984), preconceptions are either confirmed or validated and that an experiential situation, lived by the nurse, helps develop expertise. The role of recorded past experiences assists in recalling similar situations whose inferences can be applied to the new situations. This is a useful skill for nurses. The ability to associate past experience with a current situation can provide more effective care that meets the unique needs of each patient. Phenomenology necessitates discretion between the labeling and describing of a patient's experience. Both realism and common sense emerge once a grasp of human experience occurs. People live forward and must reflect 
backward upon an experience to verbalize what they had lived through (Oiler, 1982). Because this it true, it is precisely why the phenomenological question of concern has to be stated in such a way as to accurately direct the course of the participant's recollection, back to the event itself (Farber, 1968). To comprehend this process, the researcher must guide the individuals toward reflectiveness about the phenomenon and its essences. Humankind experiences and is always challenged to discover the ideal world, one that allows for self awareness via human interactions (Frost, 1962; Meleis, 1991; Paterson \& Zderad, 1976; Sahakian, 1968).

According to Watson (1979), phenomenology considers the holistic person in the context of either an experiential environment or a world that establishes meaning. She illustrated the role of suffering as a phenomenon whose observation and description by those involved could yield specific caring activities to help the individual patient proceed through the event in a unique manner. Few studies explore the role of the nurses's experience in the caring environment.

Exploring human experience from the native's perspective is necessary in cross-cultural research, Merleau-Ponty's (1964) statement "I will never know how you see red" (p. 100), provided evidence of the inability of outsiders to step into the culture of one who is living the 
experience. "The uniform cultural label helps people to understand one another in general outlines, when expressing certain experiences" (Van Kaam, 1966, p.313). Life's meaning is understood through relationships among people. Each person gives the world unique meaning and the process is reciprocal. In nursing research, quantitative designs are essential yet do not always answer the human questions sought out by members of the profession. Phenomenology seems to have filled the role of searching for the humanistic answers (Anderson, 1991). Bergum (1991) noted that phenomenological research in nursing maintains the humanness of each client and makes room for the nurse as researcher and partner. An increasing number of nursing theorists are convinced that some phenomena can only be understood qualitatively. Consequently, this philosophy has a legitimate correlational link between the profession, its nurturing nature and humanistic foundation (Silva \& Rothbart, 1984).

The goals of phenomenologic inquiry in nursing are to understand the experiencing person, to provide data so that nurses can develop as "knowers" in what Buber (1958) called the I-Thou" relationship and to present the humanistic demeanor of nursing (Burns \& Grove, 1987). In exploring the role of culture, Scudder and Mickunas (1985) explained that people do not relate to the culture but as participants in its essence, thus rendering it a researchable phenomenon. 
Describing personal experience mandates reflection, or a looking back at whatever was. As the researcher listens to participants, the former relives personal and comparable experiences. This experience of reliving establishes the concept of the "thou" as the "other I" (Schutz, 1967, p.114).

Beliefs, Attitudes and Experiences As Influences in Human

\section{Interactions}

Eishbein's Theory of "Beliefs about an object and Attitude toward the object" (1967) was reviewed to understand how beliefs about people effect attitudes toward them. The researcher also wanted to understand what occurs when people interact with one another and do not share a common ethnicity. Fishbein's theory represents the formation of attitudes about people based on personal beliefs. One of Fishbein's preliminary studies, in 1962, explored determinants of ethnic stereotyping and racial prejudice. The study was a retrospective look at beliefs that served to categorize individuals in one group or another, and attitudes formed toward particular ethnic and social units. According to Fishbein (1967), the relationship between beliefs and attitudes is never static but is continuously altered by life events which shape the view of others. Shared commonalities in ethnicity, values, health beliefs and practices may positively enhance the 
caring relationship while dissimilarities may negatively alter it. If the patient's ethnic health beliefs are in conflict with the nurse's, the patient is at risk for being labeled in negatively stereotypic terms. Caring for, living with and observing a variety of ethnicities enhance the careseeker and caregiver dyad. The more that is known about ethnicity, the greater the opportunity for nurses to understand and appreciate the uniqueness of each client (Leininger, 1977).

The progression of events has been described in this manner: beliefs are antecedents to attitudes and result in certain feelings and behaviors based on these attitudes (Cacioppo, 1981; Rosenberg et al, 1960). Beliefs about certain segments of the population are based on either favorable or unfavorable attributes (Gajendra \& Bagley, 1988). In addition to these attributes, a group's credibility in the observer's eyes, its degree of effect and the level of the observer's persuasibility influence the entire process (Petty \& Ostrom, 1981). As beliefs and attitudes are established, so are expectations about personal and group behaviors associated with the observed that often become the basis for stereotyping. Baron and Byrne (1984) presented the example of the belief about redhaired women as "hot-tempered" which was associated with Irish women but developed into a behavioral expectation of these women every time they were encountered. 
Attitudes are born out of social learning, experience and modeling by significant others. A cognitive schemata refers to a mental framework that functions as an information repository for processing and integrating data about the self, others, places, events, roles and social groupings (Baron \& Byrne, 1984). Some critical attitudinal determinants associated with people are: physical traits, such as, skin color, hair, eye and lip shapes, body odors, physique, social acceptability, mores and group behaviors (Doob, 1967; Fishbein, 1967; Helmreich, 1982; Miller, 1982). Each time a person is encountered, a like response occurs within the perceiver, thus anticipatory reactions are established and become part of the person's interactive schemata when placed in similar situations (Fishbein, 1967).

As phenomena, attitudes are considered to be evaluative responses toward people based on beliefs about them. Attitude formation is a two-step process: the object becomes the stimulus and the attitude equals the response (Fishbein, 1967). Encounters or experiences contribute to the process of encoding which creates a network for remembering past events (Petty \& Ostrom, 1981). According to Smith (1982), direct experiences encompass those events that occur through the senses while vicarious experiences are based on events outside the personal realm but exert influence on beliefs and attitudes. Several critical beliefs were identified as determinants of attitudes. They may be physical or 
psychological attributes, social acceptability, mores and behavioral norms of a group (Fishbein,1967).

Within individualist societies, such as the United States, attitudes and behaviors are usually based upon individual goals. In contrast, common societal goals are established in collective societies such as in religious sects and communistic societies (Gajendra \& Bagley, 1988). An example might be the difference in attitudes and behaviors toward Jewish citizens in an Arab nation compared to those in the United States. Beliefs about a target population are determined on its favorable or unfavorable attributes (Gajendra \& Bagley, 1988).

Basic to attitudinal decision-making is the credibility of the object source, its degree of influence upon the evaluator, persuasibility and readiness of the latter to understand the object source (Petty, 1981). Many sociologists and psychologists ask the question, "what events cause attitude development?" (Sarason, 1972). Social experiences, which assist in processing information about oneself and others, result in setting up rules or generalizations about objects, persons or events. They are the primary contributors to integrating attitudes along with one's own feelings, beliefs and world view.

Schwartz and Lacy (1982) explained that experiencing stimulates people to integrate specific attitudes. Some stimuli are events in the environment, education, rewards 
and punishment and the feedback received from others. Social events communicated through the media also affect attitudinal decision-making, as demonstrated by the poll trends (Sarason, 1972). Living in close proximity to a particular group can be responsible for imprinting attitudes. Other variables that act as attitudinal stimuli are personal needs, group affiliations, education, educators, and interpersonal communications. A process of sensitization to an object, through anxiety-provoking events may also cause variations in attitudes (Sarason, 1972). When integrating the ethnic and cultural dimensions of people, the tendency is to submerge the perception of the person as unique and personify the individual "...as a symbol we have learned to look down upon" (Fishbein, 1967, p.33).

\section{Labeling or Stereotyping as a Process}

In interactions between people, one person subconsciously categorizes the other into a particular class, based on integration of past information or experiences. Maslow (1970) referred to this process as "rubricizing". Each class is considered a rubric thus discounting the uniqueness of each human being. In 1922, Walter Lippmann, a political journalist, coined the term stereotyping as the imprinting of "pictures in our heads" about people and social events (p.92). He reasoned that a 
person's perceptions about another and the environment was not exclusively dependent on direct information or observations but also on mental images conjured up through indirect experiences. Labeling and stereotyping have been used interchangeably. The process is a fixed conception of a person or group, an attachment of a set of beliefs about personal attributes assigned to a group and representative of each member. It is a process of symbolically setting up an image of a class of individuals through assignment of specific characteristics so that each member is perceived as possessing these traits (Guralnik \& Friend, 1962; Neufeldt, 1989; Taylor, 1981).

Although often stated in the pejorative sense, stereotyping not only assigns a label but becomes an oversimplification of the group's members. Bierhoff (1989) provided examples, such as, the popular belief about Jews as clannish and ambitious, African Americans as musical or athletic, or professors as absent-minded. The image of the person becomes evaluative by virtue of belongingness to a social unit. In interactions between people, one person subconsciously categorizes the other into a rubric based on integration of experiential information (Maslow, 1970).

Within the process of stereotype formation are three components, the cognitive, affective and behavioral. The cognitive component is made up of beliefs about a particular group, the affective portion is the feeling dimension and 
the behavioral becomes the manifested component of the process (Baron \& Byrne, 1984) Frequent contacts with individuals and groups afford opportunities to acquire and process additional information that either negates or reinforces labeling or stereotyping. However, first impressions cannot be discounted because they are instrumental in label formation and perpetuation (Gallop \& Wynn, 1988; Taylor, 1981).

Goldstein (1981) pointed out that concepts such as labeling, classifying and characterizing are significant in the process of learning as the brain sets up rules based on impressions of people and events. The process is useful in labeling within phenomenological inquiry and is known as the "freezing" of personal experience into a unique occurrence.

Stereotyping is, as labeling, a generalization of an observer's perception about a class of people. The term encompasses the manifested perceptions of social beliefs, a prejudgment which becomes a mental prototype of a social class. Both behavioral and psychological dimensions are then attached to the groups (Ashmore \& Del Boca 1981; Hamilton, 1981; Miller 1982). Albeit, there are few derogatory consequences to favorable stereotypes and labels, the reverse is true about the negative ones which tend to be more tenacious and rigid. The latter remain barriers to intergroup coexistence and peace. Prejudice ensues from the observer's cognitive domain and leads to expected, negative 
behaviors each time the stereotyped is encountered (Baron \& Byrne, 1984; Bierhoff, 1989). When the connotation is positive, it is well appreciated by the identified population, ie. French men as great lovers or Italians as being strongly family-oriented (Bierhoff, 1989; Fiske \& Taylor, 1984; Tedeschi, 1984).

\section{Acquisition of Stereotypic Views}

Stereotyping is learned by virtue of one's cultural and social membership. The process occurs during socialization and functions as a decision-making mechanism that establishes the social norms of human interchange and the sanctioned treatment of one another. There is widespread agreement among writers and researchers of stereotyping that the concept encompasses generalizations about human characteristics and behaviors that set up expectations each time nembers of a particular group are encountered. Children learn, in part, through discriminate learning. This process sets up mental classifications of similar items and drives human responses to each (Forman \& Sigel, 1979). Early on in the cognitive learning, a child might perceive two or three animals or objects as part of the same class. By repeated exposures to these animals, the child begins to notice distinct features through visual learning and auditory input. Ultimately, the animals are classified into distinct categories (Forman \& Sigel, 1979). From a positive 
standpoint, stereotyping can be instrumental in early education about the world. However, children also learn both positive and negative labeling primarily within the family structure (Allport, 1958).

Lippmann (1922) wrote that the world is revealed to a person prior to it being experienced. Additional data and exposure act as stimuli that change the preconceptions. Perceiving people or events prior to the experiential encounter is a basic element in learning. Supporting this premise are the writings of Merleau-Ponty (1964) who described perception as the primary reality established by individuals. Observer bias is also influential in stereotyping people or classes by watching behaviors and expecting them to occur (Taylor, 1981). If a behavior has not been commonly associated with an individual and is acted out, the perceiver may conclude that it is an exemplar of the class to which the perceived claims membership. This is known as an illusory correlation. When an atypical behavior is noticed by a member of another homogeneous group, the observer may then posit that the act is prototypic of all the group's membership. In this circumstance, stereotyping may be the result of an inductive correlational link between the observed and the representative class (Hamilton, 1981; Taylor, 1981).

Pettigrew (1981) further explored the role of observed behaviors as an antecedent to stereotyping. The dominant 
group attaches a behavior to the non-dominant class's predisposition toward an act, and disregards the possibility of some genetic or environmental causation. A sense of control loss may also be a contributory stimulus. In the hospital setting, patients sense varying degrees of control loss and there is a tendency to respond to this stressor in a variety of ways. This process is referred to as a "causal chain of events or reason" (Fiske \& Taylor, 1983). Responses to the loss may be demonstrated as displays of helplessness, belligerence, anger, hostility and physiologic responses such as hypertension. The nurse observes the responses, draws inferences from the patient and applies them to the entire category to which the patient belongs whether the classification is an ethnicity, diagnosis or disability. The outcome of such stereotyping results in nurses anticipating like responses each time these particular segments of society are encountered (Fiske \& Taylor, 1983; Roy, 1976).

Displays of emotion, mood and the level of motivation are behavioral components upon which labeling also develops and the reasons for the above are discounted (Wyer \& Srull, 1984). Interpersonal impressions emerge from the answer to the question, "Why is this person behaving in this manner?". As a consequence, the action is either associated with the person's make-up or the environment in which the individual lives and interacts. If the behavior is defined as being 
person-related, stereotypic expectations may be imprinted each time like persons are encountered (Tedeschi, 1984).

\section{The Question of Ingroup/Outgroup Membership}

Although children learn stereotyping within the family structure, as reported by Allport (1958), stereotyping can also be formed by classifying differences between groups via personal perceptions and intergroup contacts. Observations of solo members of a group allow conclusions to be drawn, not about the individuals alone but about their influences or actions within a particular system. In these cases, the observations are solely on the group, not on the individual (Austin, 1979; Bierhoff, 1989; Gilbert, 1951; Hewes \& Planalyp, 1982). The categorization of people into particular rubrics based on certain characteristics is critical in the formation of group stereotyping. Ingroup and outgroup interactions allow for the building of repertoires about others (Austin \& Worchel, 1979; Wegner \& Vallacher, 1977). The nature of contacts between social units determines how each will be perceived. The Black/White relationship was founded on historical events which have bolstered stereotyping of all Blacks dating back to the days of slavery in America and born on the southern plantations. The system of slavery was founded on the stereotypic view of the Black race as possessing the characteristic of subservience. Of interest to note, is the fact that Blacks 
were considered to be content with their lot in life because they never overtly displayed anger toward their owners. In reality, it was fear that generated the demeanor of contentment (Stephan \& Rosenfield, 1982). Some Whites, on the other hand, knew that what at face value appeared to be happiness was probably covert hostility, engendered by the treatment the slaves received at the hands of their masters. Since then, many have stereotyped African Americans as hostile and suspicious (Stephan \& Rosenfield, 1982). Stereotyping of laborers also fostered the state of ingroup/outgroup communications between Blacks and Whites. Laborers were considered to be ignorant, physically strong and second class citizens and Blacks were members of this class (Miller, 1982). Whites were perceived by Blacks as deceitful, cunning and intelligent because the majority owned the plantations and the slaves. Because of these historical views perpetuated by each class, reciprocal stereotyping has continued (Stephan \& Rosenfield, 1982).

Likeness offers membership into an ingroup. Conversely, the fewer the similarities, the more the out of group members will be stereotyped (Kearney, 1984): Categorization of groups by ethnicity, culture, sexual preferences or other characteristics sets up a conceptual framework by which each group is envisioned. The mind then pictures the unit as homogeneous and consequently associates categorical attributes to each member (Lippmann, 1922; 
Miller, 1982). Ethnic stereotyping is often rooted in group traits, such as, skin color, physical features, approaches to healthcare and psychosocial aspects directly observed, historically linked or vicariously experienced (Helmreich, 1982).

\section{Living Proximity Influencing Social Interactions}

Living in close proximity to a particular social unit can be responsible for imprinting stereotypes. Situational variables encountered in the social environment enhance the visual and verbal development of children. They learn to make comparisons between themselves and others, form perceptions and expectations about people which guide their interactional styles (Sarason,1972; Widaman \& Little, 1985).

Social scientists have measured the levels of intimacy within which one class will admit another into its personal domain. The more intimate the spatial and relational levels, the less apt these individuals are to practice stereotyping toward a particular social segment (Bogardus, 1967). Of importance to note, are the elements affecting this phenomenon, namely, religion, race, ethnicity and occupation of the observer (Triandis \& Triandis, 1967). Social and economic competition between segments of society tend to devalue one element over another thus causing stereotyping toward the devalued person or class (Baron \& Byrne, 1984; Orque, Bloch \& Monrroy, 1983). The environment 
in which relationships occur can also be considered as a contextual stimulus (Lippmann, 1922). Environmental factors are so powerful that they can convert a human being into a "pawn on a chessboard" (Tedeschi, 1984, p.245). If environmental variables are considered as the root cause of the behavior, the person is not held responsible, consequently, stereotyping does not result. Labeling can also be an outgrowth of historical conflicts between societal units or the sociocultural milieu of the era. This process is influenced by the psychological dynamics between the observed and the observer through the cognitive process of socialization (Pettigrew, 1981).

\section{Social Forces as Contributing to Labeling}

Causation and perpetuation of labeling has been reported frequently through history. Allport (1958) examined the plight of the Jews in Europe prior to and during World War II. He stated that Jews were perceived in the same manner as the African Americans have been in the United States. Jewish people were considered to be sexually promiscuous, dirty, violent and the disseminators of disease. This perception of the race continued throughout Hitler's reign and became the reason for the extermination horrors. Gordon (1975) explained that the Jews were a minority without power which potentiated the genocide mentality of those in power positions. 
Political, economic and social conditions of a particular epoch also affect the subject and extent of stereotyping. A nationality, race or class can be labelled both negatively and positively within a short period of time (Helmreich, 1982). In the United States, the example of the Japanese internment can be presented as support for this premise. In 1941, after Japan bombed Pearl Harbor, all Japanese Americans were rounded up and incarcerated in camps here in this country, specifically in Lone Pine, California. The camp was called Manzinar. The reason for these camps was to maintain close surveillance over these citizens who by then had been stereotyped as subversives and a potential danger to the country's security. Thirty years later, the Japanese Americans were viewed more favorably because of their accomplishments in industry and technology (Estes, 1978; Helmreich, 1982; Parsons, 1975).

Beliefs, attitudes, behavioral influences, group membership, environmental and social realities form a frame of reference that affects actions, reactions and interactions with others (Wegner \& Vallacher, 1977). This framework acts as one predictor in explaining human behavior based on an organized set of experiences by observers (Stephan \& Rosenfield, 1982). From the observer's perspective, the process is influenced by a person's world view, social culture and group membership. Perceptions of the ruling class will be quite different than those of the 
subjects in a society (Kearney, 1984). In essence, people judge others by how similar they are to themselves.

Significance of Personal, Relational and Social Contacts

One of the most powerful vehicles for the creation of personal labels is in the degree and quality of contacts between people. Stephan and Rosenfield (1982) have studied and reported their research observations regarding the establishment, the nature and the results of stereotyping in their studies involving various ethnic groups. Their earliest observations involved Afro and Anglo American preschoolers. The children were presented with black and white colored dolls and were asked to choose which dolls were considered as "bad" dolls and "good" dolls. The results demonstrated that the Afro American children chose the black dolls as "bad". The researchers' findings then were interpreted to mean that Afro Americans negatively perceived themselves because of low self esteem and negative self reflection. This particular study has been rejected by some sociologists who are not convinced that preschool children can perceive a black or white doll as representative of themselves. Critics have stated that self reflection usually develops at the grade school level and encoded by media information and familial messaging (Stephan \& Rosenfield, 1982).

Stephan and Rosenfield (1982) continued by arguing that 
ethnic stereotypes tend to be more inaccurate than other forms such as those of gender or social status. Bierhoff (1989) explained that stereotyping has been operationalized on both personal and social levels. Personal stereotyping is based in individualized perceptions of some observers and does not have the same impact as social stereotyping. The latter assigns characteristics to a particular classification which is then considered inherent to all the members (Bierhoff, 1989). Not only do impressions of others become unwavering, they create mental patterns in which individuality is lost. Often, these perceptions are exaggerations of beliefs and are utilized to justify conduct in support of, or against individual members or societal units (Allport, 1958; Bierhoff, 1989; Guralnik \& Friend, 1962) .

Research into the Relevance of Stereotyping The literature is replete with documentation of the consequences linked to the process of stereotyping. Lippmann (1922) explained that the most pernicious consequence is prejudice. Once established, it is painful to surrender because the one possessing this mind-set develops a connection between the beliefs and personal selfconcept and self-respect. Deindividualization follows and gives rise to negative beliefs about a target population. The stereotypic view becomes the rationale for aggression 
against a population; once the opportunity to learn more about individuals is subjugated by stereotyping, the less importance is placed on their social role and worth. Once a negative stereotype is earmarked toward a social unit, positive behaviors carried out by its members are discounted and considered anomalous (Allport, 1958; Hamilton, 1981; Pettigrew, 1981; Wilder, 1981).

Bonaparte (1979) studied the attitudes of 300 nurses from four ethnic groups (Anglo, African American, Jewish American and Hispanic) toward culturally and ethnically diverse patient populations. The study design was quantitative and the tool used was the Cattell 16 Personality Factor Questionnaire and the Rokeach Dogmatism Scale. Her findings suggested that those nurses who were more open-minded and less ego-defensive possessed more positive attitudes toward patient's ethnicities. The author stated that care can be compromised by nurses who harbor negative attitudes toward ethnically dissimilar patients and may result in a negation of the patient's culture and health views. Bullough and Bullough's research (1982) identified that assignment of certain diagnoses connoted both social and ethnic stereotypes. The poor were more frequently labelled as psychotic as opposed to the neurotic label affixed to the middle and wealthy classes. The Bogardus Social Distance research, in 1967, measured social distance deemed acceptable by members of a 
group toward 30 ethnic groups. The tool design was a seven item scaled response. Research participants were asked to mark the responses that most closely identified their attitudes toward the ethnic groups listed. Statements, such as, would have as a friend, would marry, would not want as a neighbor and would not allow in this country were included. More participants rated the British as those they would most likely become intimate with and the Turkish as the least likely to be allowed in this country. The researchers found that the preferential ratings were reserved for those perceived to have the greatest similarities to the raters. This was a seminal study that has been replicated with varying subject groups. A literature search of nursing studies did not find one research study in which the Bogardus tool has been utilized.

In the Katz and Braly (Fishbein, 1967) study, Princeton students $(\mathrm{N}=100)$ were asked to assign various characteristics to 10 ethnic groups. The subjects were told that the traits had to be representative of the race or ethnicity, at least in the minds of the students. The characteristics most frequently associated with each class were as follows:

$\begin{array}{lll}\text { African Americans } & - & \text { superstitious } \\ \text { Jewish Americans } & - & \text { shrewd } \\ \text { German Americans } & - & \text { scientific } \\ \text { Italian Americans } & - & \text { artistic }\end{array}$


of the 10 ethnic groups included in the study, only three were assigned the trait of "physically dirty", the African Americans, Italians and the Turkish. The study results were representative of the ethnicities that were more frequently stereotyped during that particular era.

The existences of the "kernel of truth" and the "selffulfilling prophecy" have also been researched and reported in many sociological treatises (Hamilton, 1981; Helmreich, 1982; Hewes \& Planalyp, 1982; Miller, 1982; Tedeschi, 1984). Dr. Fishberg (Helmreich, 1982) explored the stereotypic belief that all Jewish people had large, hooked noses. He assessed 4,000 Jews and found that although this characteristic was present, it was only evident in $14 \%$ of those examined. This remains an example of the "kernel of truth belief" which is explained as the presence of an element of truth about certain traits yet are perceived as existing throughout a particular unit of society.

Brigham and Williams examined the concept of "crossrace own race recognition". The sample consisted of Black and White store clerks. The findings were supportive of the fact that the more frequent the contacts with members of a particular race, the greater the recognition of the individual and the less the belief in the myth that "all members look alike" (Baron \& Byrne, 1982, p.279).

Tedeschi (1984) explained that if a member of a group is lazy or unkempt, the consequence may very well be that 
all class members will be perceived in a like manner. Unfortunately, the observer will not examine the causes of the behavior or trait but will instead continue to perceive the entire group in the same light without making allowances for a possible lack of education or other factor. Stereotyped members begin to believe the perception of others. They take on the attitude that if someone believes they are those things, it must be true, therefore they begin to act accordingly, thus perpetuating the self-fulfilling prophecy. Gallop (1987) observed that the scenario is played out in clinical settings. Labeling is not based on reality but on examples set by one nember of a culture or ethnicity. Because these perceptions are often grounded in inaccuracies and fictive information, the targeted person's self-image can be destroyed. The behavior of a client may be an exhibited defense mechanism if familiarity with a setting is replaced with the unknown. Often, this occurs once an individual assumes the role of patient (Lippmann, 1922; Roy, 1976). When nurses encounter ethnic patients with whom they have had limited experience, they operationalize responses based on vicarious information, leading nurses to be resentful, inappropriate, rude and without a sense of control (Gallop, 1988; Roy, 1976).

Gallop (1988) conducted a qualitative study of registered nurses $(N=37)$ to explore their views about patients who were stereotyped as difficult. A semantic 
analysis of the interviews yielded several cogent facts. A theme developed in which nurses identified feelings of control loss in the nurse/patient dyad. Ninety percent of the nurses affixed labels, such as, "manipulative" and "attention-seeking" to describe these clients (p.38). The researcher concluded that members of the nursing profession prefer those patients who are classified as stoic, cheerful, compliant and cooperative and that there exists a common tendency for nurses to label psychiatric or chronically ill patients as combative, manic or other more derogatory names (Gallop, 1988; Kleinman, 1988). In May's study (1990), the results identified the nurses ability to classify patients as "disruptive" according to some criteria known only to the staff and then continue employing this label rather than exploring the holistic context in which the patient operates.

Fisher's (1989) qualitative study of "dangerousness" among the mentally ill demonstrated the effect of labeling clients by nurses as potentiating self-fulfilling prophecies. Affixing levels of dangerousness to patients was described as potentially being responsible for altering the latter's self-concept even though these labels were outgrowths of the nurses' perceptions and not always accurate. Fisher (1989) explained that labels had a role in facilitating interactions within the patient/nurse dyad because the nurse altered her interactions to suit the 
former's level of dangerousness, thus supporting Lippmann's "economy of effort" premise. Fisher (1989) proposed that classifying clients in this manner could also act as nursing's exertion of control over clients. In her words, "...the decision that someone is dangerous profoundly influences the person labeled, is difficult to alter and likely functions as a self-fulfilling prophecy changing forever the course of patienthood" (p.63). There are constant evaluations occurring in human communications which launch actions and responses toward people. The meaningfulness of each situation determines actions toward others. Nursing places a strong emphasis on the patient's history of mental illness, the nurse's own personal experience with similar patients, thus altering the assessment of the current situation (Fisher, 1989).

Lowenstein and Glanville (1991) conducted a limited qualitative study ( $N=12)$ of African and Anglo American nurses working in an acute care setting. Those selected were asked whether they believed that ethnic stereotyping and prejudice existed in the treatment of patients. Ninetytwo percent stated that it did. In this same study, the African American nurses also indicated that there was prejudice shown toward them as they believed that it was easier for supervisors to converse with the Anglo nurse than it was with the Black nurses because the supervisors were predominantly Anglo. In the Los Angeles Immunization Study 
(1991), qualitative data was obtained from Hispanic parents ages 20 to 40 years old $(\mathrm{N}=25)$. They were asked to discuss their knowledge about immunizations and to identify barriers in seeking vaccinations for their children. Themes such as, "prejudice against us" and "treat us badly" emerged. Discourteous behavior, disrespect and unfriendliness shown by the nursing staff were considered some of the most critical barriers that caused the participants not to return to a particular healthcare setting for follow-up. All those interviewed believed that the nurses' behaviors were based on the parents' ethnicity. One father commented that he was treated as poorly by the staff as he had been by the Immigration Department.

\section{Consequences of Mental Images}

From the observer's perspective, once a stereotype or label is ingrained in the person's belief schemata, there begins a pursuit to prove the stereotype is valid, especially associated with ethnic and racial intergroup contacts (Fishbein, 1976; Miller, 1982; Pettigrew, 1981; Snyder \& Swann, 1978; Stephan \& Rosenfield, 1982; Taylor, 1981). A powerful example might be the perception of Hispanic Americans as indolent, unkempt or unreliable. These characteristics were associated with the laborer class and not initially with the ethnicity itself. Over time, a link developed between the individual and the trait in what 
is known as consequential stereotyping based on social schemas (Fiske \& Taylor, 1983).

Goffman (1963) further supported the consequences of labeling by stating that once individuality is subjugated, the person is seen in light of a particular class. This event restricts actions against and interactions with that person. The key concept is that labeling destroys individuality, submerges the person into group identity which is perceived either positively or negatively. The emerging portrait then becomes the basic truth upon which actions among people are created.

Although individuals involved in a communication setting may be inherently dissimilar, they share some commonalities. The key is to explore the common ground because the major cause of prejudice is based on psychosocial elements that are viewed by people as not being congruent or the "...inability to accept something different as equal, and the inability to adapt" (Gudykunst, 1983, p.223). Economic and social competition, social categorization or the devaluation of one unit over another are also responsible for prejudice formation (Baron \& Byrne, 1984)

According to Orque, Block and Monrroy (1983), attitudinal negativity toward ethnic groups exists in nursing. Lack of knowledge about ethnically and culturally diverse patients contributes to inappropriate and inadequate 
patient care. These patients are often treated as though they have few feelings, fears or needs and nurses respond without sensitivity (Henderson \& Primeaux, 1981). The greater the differences between the caregiver and the careseeker's health beliefs and practices and the less information is known, the greater the incidence of rubric generalizations (Bawwens, 1978; Leininger, 1978).

All healthcare providers are themselves ethnic and are members of a cultural system which allows for a measure of self-perception. Conflicts occur frequently when both patient and nurse have no common cultural linkage. Stereotypes shape both attitudes and the care encounter either negatively or positively (Smith, 1982; Spector, 1979). Roy (1976) asserted that not only does prejudice bias the attitudes of people against each other, and nurse against patients, but also affects the victim's self-image. Baron and Byrne (1984) explained that an unexpected resultant of prejudice or stereotyping is a self-fulfilling prophecy in which the victims of the bias develop low selfesteem, altering their behaviors negatively which tend to reinforce already established attitudes held by the observer.

\section{Interethnic Relationships between Nurses and Clients and} Unifying Factors

In 1923, the Committee for the study of Nursing 
Education identified the importance of educating nurses about social and economic influences that affected the health and illness status of people. Language continued to be the most common barrier and the report encouraged nurses to develop familiarity with the language of the clients they served in order to build a bond between both of them. This same report also suggested that nurses needed to develop an understanding of certain aspects of ethnicity such as myths, diet, habits and healthcare systems. Bridgeman (1953) understood the importance of introducing sociology and anthropology into the nursing curriculum as a means of assisting students in developing insight into societal origins, exploring human problems and formulating cultural views. For Branch (1976), understanding the ethnic and cultural practices of clients prevented the discounting of beliefs and practices that are viewed as efficacious by the members of a unique group. DeTornyay (1976) recommended that nurses develop an appreciation of a person's creed and ethnicity to provide quality nursing care in a culturally sensitive way.

Carter (1991) suggested that understanding the values of other ethnic groups helped individuals understand their own values and how they influence behaviors. Elements such as, language, dress and experience do have direct effects on personal interactions. In order to enhance these communications, learning the histories and values of people 
can become the foundation for appreciating each person's uniqueness. For most, their histories are replete with oppression and racism. According to Carter (1991), a familiarity with another's roots enhances a person's worldview. Each person needs to become aware of those "cultural filters" through which beliefs, feelings and behaviors are perceived (p. 172). This allows for selfreflection about the ethnic and cultural heritage that is part of the personal schema, thus building an appreciation for humankind which is "at a deeply personal and emotional level" (p.172).

Baron and Byrne (1984) suggested that the positive effects of interethnic communications serve to stimulate the discovery of similarities with others rather than concentrating on differences. These effects enhance attraction for those considered as non members of a particular group. They promote a sense of community and decrease the continuation of prejudice and stereotyping. In addition, Orque, Bloch and Monrroy (1983) explained that nurses must maintain an awareness about how various groups respond to pain, grief and other life events so that care can be applied in a sensitive and empathetic way. As Baron and Byrne (1984) summarized, "the recipients of medical care want to be liked and to be informed. Factors that increase a sense of responsibility, control, and self-reliance tend to have significant effects on feelings and behavior" ( $p$. 
514). For nurses, becoming more knowledgeable about the ethnicities and cultures of people not only affects the quality of communications but also allows for a more positive and valuing care experience for each client.

\section{Interactional Dynamics}

The search for clues to the essence of the interactions between the ethnically dissimilar nurses and clients in this study became critical as the participants were interviewed. Emerging themes such as uncommon languages as a barrier, nonverbal cues and their influence on the interaction seemed to affect the process that occurred between nurses and clients and needed further explanation and validation.

Although the mechanics of labeling or stereotyping were identified in the literature review, the need to reach beyond the initial literature search seemed imperative as the participants mentally assessed their clients and labelled them based on physical features, class, address and lack of English fluency. Feelings of challenge, trust, acceptance, satisfaction, inadequacy, frustration and anger were also identified by the nurses in this study as well as nonverbal cues that became a key component in situations when language was not shared. Finally, there seemed to emerge, from all of these perceptual and sensory processes, a dyadic relationship that became workable. 
Language Differences and Paralanguage

Arnold and Boggs (1989) stated that the inability to communicate via a common language poses severe constraints on the individuals in each interaction. However, a common language spoken in the nurse/client dyad does not insure understanding in all cases. There may also be misunderstandings due to word meanings and implications that are not known to the two parties. The label "Paralanguage" (p. 16) incorporates intonation, verbal expressions and pitch that may alter the communication process with others. Nonverbal communication, which includes gestures, body and facial expressions may also inherently affect the quality of an interaction. Arnold and Boggs (1989) compared the reliability of nonverbal and verbal communication. In their opinion, the former is more "reliable" (p. 18) but can more easily be misconstrued. They suggested that nurses remain alert to the non verbal cues, especially in identifying feelings of pain and mental state, such as depression and anxiety.

Facial expressions encompass the covert feelings of an individual. They allow for the creation of perceptions. Facial expressions act as a "barometer" of a person's personality and interactive style. They serve to create realism between feelings and expressions. The facial expression "... either reinforces or modifies the verbal message received". Nonverbal messaging supersedes the 
expressed word (Arnold \& Boggs, 1989; p. 19). Both verbal and nonverbal expressions are interactive and reciprocal so that the nurse can message the client and vice versa. Eyes, eyebrows and the mouth are the features that are most telling and less controllable. Although false assumptions can be made, based on verbal and paralanguage cues "...it is impossible to communicate these assumptions". In communications, Buber's (1958) notion of the "I and Thou" is operationalized (p. 22). To identify the power of facial expressions and words, Mehrabian (1971) created a formula that identifies this relationship:

$$
\begin{aligned}
& 55 \% \text { facial } \\
& 38 \% \text { vocal }
\end{aligned}
$$

All three form the total sensory expression (p. 77).

\section{Dress and Body Adornments as Cues}

During the interviews, the participants ( $N=15)$ mentioned that the client's personal appearance and dress stimulated assumptions about the person's ethnicity. Helman (1992) wrote that the body also has a "social" role in how people are viewed (p. 12). This is an inherent part of the communication encounter. Clothing is one criteria that affords the interviewer the opportunity to make assumptions about "...social rank and occupation" (p. 12). Some examples were the wearing of jewelry and fine clothing that may signify wealth. Use of a white uniform or laboratory coat signifies a professional person. Body piercing, 
tattooing, orthodontics, hair and fingernail care also serve as cues about the person in the eyes of the beholder. In this research, body adornment helped the nurses assess the social class, wealth, and possibly even the level of education attained by their clients. One participant shared that her client was well dressed in "Nordstrom" type clothing, hair was "coiffed", fingernails manicured and she also wore lovely jewelry. She appeared to be "educated and wealthy". These statements supported Helman's premise that body adornments send messages about a person's status.

\section{Awareness of Self and Sensory Reactions}

The clinician should always remain cognizant of his/her own personal, cultural, economic, educational and religious background in order to promote and maintain effective communications with each client. As a consequence, the balance of power between both may remain equalized (Helman 1992). MacGregor (1990) wrote, "Unless a nurse learns to think in cultural terms, just as she learns to think in psychologic terms, she will continue to be baffled, if not exasperated on such occasions" (p. 38). Not only can communication problems occur when two different languages are spoken but also, at times, when a common language is shared between the nurse and the client. To the extent that the American approach of being very direct with clients is not commonly practiced in other cultures, nurses need to 
understand their actions and place the patient in the context of personal ethnicity and culture, rather than labeling patients as non-compliant.

Peplau (1991) presented a schematic that identifies the trait of frustration and how that attribute may interfere with communication (p. 95). Because many of the participants mentioned the feeling of frustration, the idea of learning more about this sensation seemed warranted. According to Peplau (1991), there is a connection between frustration and feelings of inadequacy relative to the patient's goal and the goal attainment. The level of frustration experienced by one individual is not necessarily the same as for another individual. The feeling varies in degree, is dependent on personal traits of the one who is frustrated and also on the need or goal that has not been achieved. In some patients, frustration is often demonstrated against a nurse or in the lack of goal attainment. In order to overcome this feeling of frustration, Peplau (1991) suggested that there needs to occur a "transformation of energy" (p. 95). Barriers must be identified and aggression must be minimized. The goal should be to reestablish a sense of safety for both people in the dyad. Frustration inundates the expenditure of energy and can only be converted into a positive force when the experience is reviewed and understood. 
Emergence of the Therapeutic Model

In the ethnically diverse relationship between the nurse and the patient, although the participants did not identify any nurse theorists as a common premise, there appeared to be an emergence of Travelbee's (1971) therapeutic relationship model. The author described the "phase of original encounter" when each participant meets the patient and assesses a common communication ground for the interactions. For Travelbee (1971), it is a time when nurses try to set aside all prior knowledge about patients, inclusive of ethnicity, culture or diagnosis. Arnold and Boggs (1989) identified this particular phase as "the instant in which ...all previous biases or ideas of what the client should be like occurs" (p. 31). Phase two is the time when both the client and the nurse demonstrate their own personalities, a time for sharing new information about each other. This moment heralds in the beginning of actually experiencing each other. Thirdly, the concepts of "empathy and sympathy" emerge as part of the interaction. At this time, the nurse tries to understand the patient in the context of some recalled life experiences. The nurse becomes the creator of a therapeutic environment. The final phase of rapport is formed when the personal interactions take on some significance and meaning for the dyad (Arnold \& Boggs 1989; Travelbee, 1971)

Mutuality becomes the by-product of the nurse/client 
dyad when all verbal and nonverbal language is assessed, when the nurse begins to see the development of a therapeutic milieu and the responses from the patient. For the nurse, knowing the nonverbal and facial expressions of a client, when language is a barrier, remains one of the most critical components of a therapeutic evaluation.

Arnold and Boggs (1989) outlined the ethnic and cultural context in which interactions occur. The context is composed of health beliefs and practices, religion, language, verbal and nonverbal communication, roles of family members, values, rituals, time and space orientations. In assessing the patient and forming the most therapeutic and appropriate ethnic environment, nurses need to also be cognizant about how family members can assume positions within the health care environment, in relation to the patient, and become useful in interactions.

\section{Summary}

The historical development of phenomenology and its influence on nursing research was outlined. The importance of this method was described as enhancing nurses' knowledge of the holistic person thus stimulating the relevance in the development of theory and further research. Because nurses are in constant interactions with others and form both beliefs and attitudes about them, the process of labeling was discussed as a time-limited snapshot of a culture or an 
ethnicity (Helmreich, 1982).

Acquiring stereotypic views was explained as a normal process that is influenced by ingroup/outgroup membership, living proximity of one person to another, social forces, such as, personal, relational and social contacts. The ability to view each person as a distinct whole is time consuming and often impossible so that individuation is reserved for those significant people admitted into the circle of intimacy. When individuality is not considered, stereotyping of people occurs and can either positively affect relationships or lead to prejudice (Fishbein, 1967, Lippmann, 1922).

As research into the relevance of stereotyping was reviewed, its use as a time efficient way of absorbing information and maintaining it for future reference was considered in the context of an "economy of effort" idea (Lippmann, 1922). This process allows for the expansion of a cognitive repertoire associated with human attributes and characteristics, thus linking people together in a conceptual framework for anticipatory purposes. Human experience often forces alterations in this construct as additional knowledge is acquired about groups and their membership (Gilbert, 1951; Katz \& Braly, 1933; Turiel, 1983; Wegner \& Vallacher, 1977).

As observers of the world around them, humans have a tendency to cluster traits together about a given target 
population and formulate a lasting impression which is known as stereotyping (Tedeschi, 1984). The positive advantage of this process is the ability to anticipate or predict behaviors of strangers based on known data. The dichotomy is that negative stereotyping becomes the justification for prejudice which rationalizes behaviors against humankind.

As the dynamics of human interactions were explored in the literature, influences such as, language differences, non-verbal expressions, dress and body adornments were suggested as being capable of altering personal awareness and sensory reactions between individuals. Sociological and nursing research was examined to identify the presence and significance of labeling in the nurse/patient dyad. Travelbee's therapeutic relationship model (1971) was outlined in an effort to clearly understand what transpires as nurses encounter patients and assess common grounds upon which reciprocal communications can progress. The role of environmental factors that impact interactions were mentioned as creating heightened awareness of the totality of each encounter.

Interactional dynamics, such as the role of language differences, paralanguage, dress and body adornments were explored. The need to remain aware of the self and one's sensory reactions was also discussed in an effort to create a therapeutic care model when interacting with ethnically diverse clients. 
Chapter Three

METHODOLOGY

Chapter Three will include a detailed discussion of Van Kaam's research method. Each component of the process will be identified and explained. This process includes, the collection of information, a presentation of the study participants and method for their selection, the interview process, the listing and grouping of the volunteers' perceptions and feelings, major questions and the reduction and elimination of data. An explanation of the usefulness of a panel of experts to review the phenomenon's constituents will also be defined. A brief explanation of the need for hypothetical and final identifications of the phenomenon under study will be discussed. How this study's findings can be applied at a later date will be reviewed as well as the data analysis process.

Following an explanation of the data analysis process, information will be provided that explains the criteria for maintaining trustworthiness, rigor, dependability, confirmability and subjectivity, accuracy and truth and the problem of generalizability in phenomenological studies. In concluding Chapter Three, the researcher will explain how 
language expands awareness about the phenomenon as well as what strategies were established to maintain ethical considerations throughout and following the study. Because the goal of this research was to explore the lived experiences of nurses interacting with patients who were ethnically different than themselves, a phenomenological method was utilized. It was the researcher's intent to discover the true moments of these experiences while taking into account the human qualities of both the researcher and the study participants. For this study, implementation of Van Kaam's research method afforded the most succinct and structured process for the collection of information, selection of participants, categorization of information and analysis of data. This methodology allowed the researcher to gather data through interviews, classify information as it represented the phenomenon and presented the opportunity to have the data verified by a panel of judges. This latter step preserves the dependability of the information obtained and assists in affirming the findings. In this study, nurses were established as evaluators of their experiences in interactions with ethnically diverse care recipients.

\section{Van Kaam's Method}

Throughout this study, Van Kaam's method was implemented as it offered a methodology that has a basic 
structure and has been frequently utilized by nursing theorists and researchers to explicate the experience being studied. One of the most critical steps in this design is to choose a phenomenon that had been experienced by the researcher and offered interest as well. Questions pertaining to the event are then formulated to assist the participants in recalling and revealing their perceptions and feelings while living the phenomenon. Early on, the researcher's responsibility is to set aside all knowledge regarding the study event and to begin with "naive perceptions" (Van Kaam, 1966, p.296). The aim of this method is to uncover the necessary "constituents of this feeling" (p. 301) through the stories of the participants. To this end, Van Kaam's (1966) six phase method was implemented: "listing and preliminary grouping, reduction, eliminating, hypothetical identification, final identification and application" (p. 314). Although these steps are distinct, they may frequently overlap during the study process.

Description of the Methodology The methodology will be described as well as the value of the phenomenological method and Van Kaam's process. The participants will be described in terms of their selection based on specific criteria. The process of explicating the phenomenon will also be discussed as well as how the ethical 
considerations were addressed.

\section{Collection of Information}

In this study, participants were interviewed at a predetermined date, time and place. They were asked to describe their feelings when experiencing the topic and to recall a situation or situations in which the phenomenon was present. By posing questions that were designed to elicit information, the subjects were asked to describe both their feelings and their perceptions at the time. All data were collected through the use of taped interviews. Interviews and the collection of information continued until the researcher believed that no new insight or themes had emerged. Then the process of data categorization and analysis began.

After the information was collected, the researcher began to categorize or label constituents or moments in which the phenomenon seemed to be present. The data categories were based on what the participants said and were presented in their own words and statements. The end result was a hypothetical definition of the phenomenon described through the perceptions and the feelings of the participants. For Omery (1983), the analysis is found in the descriptive definition of the phenomenon. Reducing the amount of data into categories that express the phenomenon is a method of refinement that is used to construct an 
analysis.

Van Kaam (1966) conjectured that a person's knowledge of the world occurs both through experience and in the appearance of what is in the person's world. The process of forming categories about the phenomenon is a way of observing its description repeatedly. The researcher must use the descriptive terms to label the moments when the experience is perceived and felt.

As the data were reviewed, the elements of the phenomenon were categorized into perception and feeling labels using the participants key words or phrases, followed by a tabulation of the frequency of their use. All expressions by the participants were listed except those that had no relationship to the experience. A panel of experts was then requested to review the categories to ascertain whether the elements were descriptive of the phenomenon. The judges and their critiques are discussed in the section entitled "Reduction and Elimination of Data and the Usefulness of Experts" (p. 73).

\section{Participants}

Van Kaam (1966) suggested that the researcher select individuals who had not only experienced the phenomenon but were able to explain it clearly, including their personal feelings that were present at the time. They also must possess a good command of the language to be utilized in 
these conversations as well as a keen interest in the topic being discussed. Selection of these individuals, according to Van Kaam's guidelines, is considered as a "primary selection" of participants and is the most effective method in phenomenological research (Morse, 1991, p. 136).

\section{A Composite of the Participants}

Ray (1994) stated that participants need to be chosen from among those who have been part of the experience under investigation. Whether the study is descriptive or interpretive, the researcher must finally approach the "meaning of the experience", which is, sensory (p. 127). For Van Kaam (1966), an experience is not merely sensory but also creates perceptions. Of importance to note are not only the feelings of the study volunteers but also their perceptions of what this experience was like and how they described these interactions with clients who were ethnically different than themselves.

A total of fifteen participant nurses were chosen. Their ages ranged from 24 to 66 years old. Each identified herself as a member of one or more ethnic groups - eight considered themselves as "Anglo" and added an ethnic component such as, French, Irish, German, Hungarian, Russian or scotch. Of the remaining seven nurses, three stated they were "Mexican", two were "Filipino", one was "Chinese" and another "West Indian". For these participants, ten were 
raised in the United States while five had immigrated from other countries. Their educational preparation had been relatively broad, with five having received either diploma or associate degrees, five possessing baccalaureate degrees, and one with a master's degree. Four of the fifteen participants were Nurse Practitioners. All were currently employed and had a minimum of one year of nursing experience. Their areas of specialization included acute care, ambulatory care and public health. The average years of experience was sixteen. All the participants had travelled outside the forty-eight contiguous United States, some more extensively than others. Each volunteer had experienced at least one interaction with a client who was ethnically different than herself.

Only participants who had lived experiences with patients of ethnic groups other than their own were accepted as participants. Every volunteer was reimbursed $\$ 20.00$ for her interview time. This was to assist in defraying costs incurred as a result of participation in this study. Although participation did not exclude male nurses, only female nurses willingly participated ( $N=15)$.

\section{Method for Selecting Participants}

Participation was voluntary. The researcher was planning to solicit volunteers by making appearances at various organizational meetings, such as the Asian-American 
Nurses Association of California and the Sigma Theta Tau Honor Society, however, the process evolved into snowball sampling (Polit \& Hungler, 1993). Some nurses who were known to the researcher were invited to participate. They in turn, suggested other potential volunteers who had experiences working with ethnically diverse patients. Once their names and phone numbers were submitted to the researcher, each nurse was contacted. During the initial contact, prospective volunteers were asked if they had ever experienced the phenomenon in question. If they had and were willing to participate, they were given information regarding the study. A date, place and time were then established for each interview. During the phone call, the participants were also informed of the following information: the need for consent signatures, the fact that the interviews were to be tape-recorded and would last approximately one hour, all information would be confidential and that all the interview tapes would be coded in such a way so that there would be no method of matching the information with their names, except by the researcher. Fifteen volunteers were willing to participate. Twelve nurses were interviewed in their homes while three were interviewed in their offices after working hours.

Prior to the interview date, the prospective participants were asked to think about a client/clients who 
were ethnically different than themselves and try to recall these interactions in their totality. The participants appreciated having some opportunity to think about these experiences as they said they wanted to be "prepared" prior to the taped interviews.

\section{Interview Process}

The researcher travelled to the appointed location that was chosen by each volunteer. After the initial introductions were completed, each nurse was asked to read the Consent To Act As A Research Participant (Appendix B). Each was also asked again, if there were any questions about the consent. If the volunteer consented, signatures were affixed and the interviewing process was once again explained. They were shown the Demographic Questionnaire (Appendix C) and asked to complete it. The results are described in Tables 1 and 2 and include the participants: ages, ethnicities, years of experience, professional degrees earned and areas of specialization. Prior to the beginning of the taping, each nurse was told to give the researcher a hand signal if they wanted the tape turned off for any reason. They were asked to relax and to think of the client whose story they wanted to share. Once they were ready, the tape was begun. 
Major Questions and Probes

The questions were prepared and presented as noted below (Appendix D):

Major question:

1. Think about the most memorable patient or patients you interacted with who were not members of your ethnic group. Please share all the information you can about the interaction until you feel there is nothing more you can tell me.

Probe questions:

2. Tell me how you decided that the patient's ethnicity was different than yours?

3. Tell me the story: what was the situation, what occurred, where did it occur, who was present, when did it occur, what was said?

4. How did you feel?

5. What would you have wished for, what did you fear, what was the most pleasant, the most difficult, satisfying or challenging?

of importance to note in Van Kaam's method, are the outward reactions or behaviors exhibited by the participants during the conversations. These observations were recorded as field notes following each interview. 
Table 1 DEMOGRAPHIC ATTRIBUTES OF THE PARTICIPANTS

$$
\mathrm{N}=15
$$

\begin{tabular}{||c|c|c|c|c|c||}
\hline \multicolumn{2}{|c|}{ AGE } & \multicolumn{2}{c|}{ ETHNICITY } & \multicolumn{2}{c||}{$\begin{array}{c}\text { YEARS OF } \\
\text { EXPERIENCE }\end{array}$} \\
\hline \hline $24-35$ & 3 & Anglo American & 8 & $2-5$ & 1 \\
\hline $36-45$ & 3 & Asian American & 1 & $6-10$ & 1 \\
\hline $46-55$ & 3 & Pacific Islander & 2 & $11-15$ & 2 \\
\hline $56-65$ & 4 & Mexican American & 3 & $16-20$ & 3 \\
\hline $66-75$ & 1 & West Indian & 1 & $21+$ & 8 \\
\hline $\begin{array}{c}\text { No } \\
\text { Mention }\end{array}$ & 1 & & & & \\
\hline
\end{tabular}

Table 2 PROFESSIONAL PREPARATION AND AREAS OF SPECIALIZATION

\begin{tabular}{||c|c|c|c|}
\hline \multicolumn{2}{|l|}{$\begin{array}{l}\text { PROFESSIONAI DEGREES } \\
\text { EARNED }\end{array}$} & \multicolumn{2}{l|}{ AREAS OF SPECIALIZATION } \\
\hline Diploma & 4 & Acute Care & $\mathbf{8}$ \\
\hline $\begin{array}{c}\text { Associate } \\
\text { Degree }\end{array}$ & 1 & $\begin{array}{c}\text { Emergency } \\
\text { Medicine }\end{array}$ & 1 \\
\hline $\begin{array}{c}\text { B.S.N. (2 } \\
\text { N.P.'s had } \\
\text { earned this } \\
\text { degree }\end{array}$ & $\begin{array}{c}\text { Pediatrics and } \\
\text { Pediatric } \\
\text { Intensive Care }\end{array}$ & 2 \\
\hline $\begin{array}{c}\text { M.S.N. (1 N.P. } \\
\text { had earned } \\
\text { this degree }\end{array}$ & 1 & Obstetrics & 5 \\
\hline $\begin{array}{c}\text { Nurse } \\
\text { Practitioner }\end{array}$ & 4 & $\begin{array}{l}\text { Ambulatory } \\
\text { Care }\end{array}$ & $\mathbf{3}$ \\
\hline & $\begin{array}{c}\text { Family } \\
\text { Practice }\end{array}$ & 2 \\
\hline & Pediatrics & 1 \\
\hline & Public Health & $\mathbf{4}$ \\
\hline
\end{tabular}


Listing and Preliminary Grouping of Perceptions and Feelings

Interviews were utilized to collect the information and an outline of questions was prepared to assist in eliciting information and themes that were descriptive of the nurses' lived experiences (Appendix D). All interviews were tape recorded. Each tape was transcribed by a professional transcriptionist. Both the tapes and the transcriptions were returned to the researcher for processing.

While awaiting the return of all the transcripts, the researcher listened to each tape and recorded various phrases, feelings and statements that were more commonly utilized by the volunteers. Each transcribed story was carefully reviewed and key sensory and perceptual statements were highlighted. These statements were then listed into categories identifying them as either perceptions or feelings. Both the phrases and the frequency of their mention were listed. Even if at the first review, there seemed to be little connection to the phenomenon, the statements were listed. The next step was to blend the statements into sensory and perceptual themes (Van Kaam, 1966). The themes were divided into Level I (Perceptions) and Level II (Feelings). By using probe questions (Appendix D), the volunteers were guided in telling their stories until they had nothing more to discuss. Feelings, perceptions, reactions and behaviors were described. The volunteers identified challenges, frustrations, satisfying 
and learning moments in each of their interactions.

The accounts ended by participants recounting how they were affected by these encounters as well as what part of the client's ethnicity was operationalized at the time and what was the most memorable learning opportunity in their stories. Each interview lasted from forty-five to sixty minutes. All participants were eager to tell their stories and all fifteen were articulate. One nurse shared that she should pay the researcher for listening to her. Another stated that retelling her account was "very therapeutic".

Once the tape recordings were transcribed, information was listed and categorized according to commonality and frequency of the expressions. Each category was created from like responses that held the "moment of the experience" (Van Kaam, 1966, p. 305).

Reduction and Elimination of Data and the Usefulness of Experts

The listing and coding procedure was completed after each interview transcription was received. The actual words of each participant were written in the thematic groupings. When descriptions did not appear relevant to the experience, they were not eliminated but maintained for review by the panel of experts. Another useful suggestion by Van Kaam (1966, p. 47) was the inclusion of information into specific categories, even in light of the fact that only one expert 
may agree with its inclusion. In the final analysis these descriptors can be deleted or verified. The experts are then commissioned to examine the groupings in order to ascertain the "thematic moments". Van Kaam (1966) employed three psychologists to review his data in the study of "Really Feeling Understood" (p. 325) in order to maintain objectivity.

Experts can be chosen from among those who either know very little or a good deal about the research being conducted (Bergum, 1991; Morse, 1991; Van Kaam, 1966). If the goal of a particular phenomenological study is to generate theory, the use of judges who are familiar with the study is more appropriate, according to the above authors. Prior to the expert panel review, the researcher prepares questions that each can pose as the data are examined. This affords each judge an opportunity to determine whether the descriptors appear to be associated with the experience. Three nursing faculty members of this dissertation committee at the University of San Diego and a doctorally prepared phenomenologic researcher, who had conducted qualitative research in the past, were invited to review the categories. A letter, questions and an outline of the categories were mailed to them (Appendices $E \& F$ ). Included was a self addressed stamped envelope for the judges' convenience in returning the information to the researcher. The experts returned their feedback to the researcher and 
themes were clarified. The nurse phenomenologist requested as much of the data as were available. All of Chapter Four was sent to her.

Another expert stated that the perceptual categories were not as clear as all the emotional categories. The third judge did not submit any comments while the fourth reviewed all of Chapters Four and Five. All requested further information to assist them draw conclusions about the appropriateness of the themes. As a result of these critiques, additional information (transcripts) was submitted to these individuals and their helpful feedback was incorporated into both the presentation and the discussion of the findings.

\section{Hypothetical Identification}

Following the experts' review, the researcher synthesized the phenomenon's common essences or themes into a statement that became the description of the experience. In this case, the phenomenon of "lived experiences of nurses's interactions with ethnically diverse clients" was defined by using descriptors that had been furnished by the participants. Bergum (1991) stated that the researcher's own experience is critical in analyzing the participant's descriptions of the phenomenon. This hypothetical description appears in Chapter Five. 
Final Identification

The final focus in Van Kaam's (1966) method is the outlining of the various constituents of the experience through the use of perceptions and feelings described by the participants'. Their statements allow for the visualization of the phenomenon. Lastly, the researcher's responsibility is to compose a "synthetic description of the experience" as a summary statement of the participants' expressions (p. 325). The phenomenon should also be labelled as an experience rather than as a feeling because the former embodies "perceptual moments" (p. 326). Van Kaam (1966) suggested that the final identification of the phenomenon be applied to another group of participants in order to determine if new themes emerge. This step can be completed at a future date.

As a result of the numerous reviews of both the transcripts of the taped interviews and repeatedly listening to the tapes the final constituents were identified by labeling them in terms of whether the statements were representative of perceptions, which are directed toward the object being investigated or were representative of feelings, which are directed toward the subjects experiencing them (Table 3 ).

\section{Application}

Following either the final description of the 
experience or its clarification, the phenomenon should become visible to the researcher. The phenomenon is then viewed in terms of usefulness to the foundation and expansion of human understanding. What is the meaning of this experience? Van Kaam (1966) believed that "...its results are useful in problems of therapy and counseling, interpretation of personality traits, creativity, and human relationships" (p. 329). The application of this phenomenon and its explanation will depend on future participant input, thus can be replicated with other nursing groups in order to review its usefulness in furthering nursing research, improving nursing practice or education. The information learned from this study can also become the foundation for other studies and yield different statements about the experience.

Data Analysis: Process in the Identification of Constituents

In order to explicate possible perceptions and feelings of the phenomenon in this study and to reduce them to significant essences, the following twelve step process occurred:

1. All taped interviews were professionally transcribed as the researcher believed that this step was more technical than scientific.

2. Both the tapes and the transcriptions were returned to the researcher. 
3. Each transcription was carefully reviewed and each feeling and perception stated by the nurses were highlighted.

4. The tapes were played repeatedly and additional notations were written on the transcripts.

5. After the transcript review was initially completed, each feeling and perception was listed in a notebook - under the headings "feelings subject-directed" and "perceptions object directed".

6. The transcripts were reviewed several times.

7. Three faculty members were given a transcript to critique prior to the completion of the data collection to determine whether the feelings and perceptions had been thoroughly captured and whether the questions were posed in such a way as to encourage the participants.

8. After all possible constituents were listed, they were reduced into categories according to the frequency of their appearance.

9. The list of categories was then submitted to three faculty members and one additional expert for review and critique. A cover letter (Appendix E) and the list of constituents (Appendix F) were included in order to make the directions clear.

10. As the critiques were returned, the changes were incorporated.

11. The final list of constituents was then decided upon as it appears in Table 3 .

12. The next step in the process was to arrive at a hypothetical identification of the experience (included in Chapter 5).

Van Kaam (1966) suggested that perceptual moments be listed first because they are object-directed thus precede the emotional constituents that are subject-directed. Although both perceptions and feelings cannot be explicated from each other, they were listed separately (Table 3 ). 
Maintenance of Trustworthiness

Lincoln and Guba (1985) explained that trustworthiness or "truth value", (p. 290) which encompasses credibility, transferability, generalizability, rigor, dependability and confirmability, becomes manifest through a process that is made up of various steps. Marshall and Rossman (1989) based their suggestions on the Lincoln and Guba (1985) model and offered a methodology that confronts and addresses these issues. In phenomenological research, the issue of credibility can be addressed in a thorough description of the study participants through a summarization of demographics. Transferability is the term that refers to the generalizability of the findings. In order to credit phenomenological findings of one group to other similar populations, Van Kaam (1966) believed that within a particular culture, people learn and incorporate common expressions to explain a singular experience. Repetitive use of these unique labels enables people to understand each other. In this study, the categorization and review of common phrases and labels helped to identify the common language that allowed for the visualization of this phenomenon by the researcher. Although the findings cannot be generalized beyond this study's participants, the study can be replicated to increase the collection of other essences relative to this phenomenon and to make this study's findings more dependable. 
$\underline{\text { Rigor }}$

In phenomenologic research, there remains the question of maintaining rigor throughout the study. For this study, rigor was maintained by completing thorough data collection, accurate observations and in depth interviews. At issue in this method, is the researcher's ability to listen and truly understand the nurse's stories while setting aside all prior knowledge of the phenomenon. Spiegelberg (1982) referred to this step as bracketing. The researcher needs to attempt to see through the participant's experience. Once data were collected, the transcriptions were scrutinized in order to comprehend the essence of the phenomenon (Burns \& Grove, 1987; Spiegelberg, 1982).

\section{Dependability}

For Morse (1991), dependability is akin to the reliability issue in quantitative research. Van Kaam's method (1966) allows for the matter of dependability to be preserved by repetitive conversations with the participants to clarify information during a short time period lone to two months). In addition, the data review carried out by the panel of experts enhances the dependability of the findings. Both these steps were completed.

Dependability can also be established and maintained through the data collection, documentation and analysis that are truthful accounts of people's stories. For the 
researcher, ensuring that personal bias does not interfere with observations and data collection is crucial. In Van Kaam's method, bias is minimized because the data is reviewed and ascertained by a panel of judges. In assessing the data, the judges are asked to answer specific questions that are proposed by the researcher in order to look for specific essences of the phenomenon. The search for categories continues until they no longer emerge (Morse, 1991). Repetitive interviewing of the participants to validate the interpretation and the intentionality is another method to insure dependability. The phenomenon also becomes evident when the same themes emerge consistently. To maintain a sense of what the participants have experienced, it is also important that the researcher has lived the experience being explored (Morse, 1991). MerleauPonty (1964) believed that reliability (dependability) was inherent in the lived experience. Each person is the absolute source of reality within the context of a personal world. Phenomenology assists in examining and describing the primordial world of experience. This allows for the classification and analysis of the essences and meaning of the phenomenon under investigation.

Confirmability and Subjectivity

Confirmability or objectivity in data processing lies in the researcher's ability to remove self evaluation from 
the participants' conversations and findings. In phenomenology, the stating of personal assumptions and bracketing of one's knowledge regarding the phenomenon assist in preserving the confirmability of the findings (Lincoln \& Guba, 1985; Marshall \& Rossman, 1991; Spiegelberg, 1982) .

Subjectivity is established because people live experiences and describe their own perceptions of reality. Each creates a reality that is interpreted and remembered (Heidegger, 1964; Husserl, 1977). Because reality is also replicated as frequently as individuals live the experience, the limitations to phenomenology occur because the method is time consuming and therefore limited by the number of participants in the study (Van Kaam, 1966). For this study, the constituents were described by stating the essences in the participant's own words.

\section{Accuracy and Truth}

Accuracy and truth in information are confirmed once the reader of the data can state that they understand the experience and can relate to the feelings and perceptions of the participants, through the terms and labels that emerge from the analysis. Schutz (1962) explained that as members of the universe, people establish a relationship with it, thus rendering their personal experience valid. He argued that theory should be based on an individual's existential 
world that focuses on living an experience. Both Brink (1991) and Van Kaam (1966) agree that accuracy and truth become evident when there is "intersubjective concurrence" (Van Kaam, 1966, p. 315), and when the experts agree with the data categories or themes identified by the researcher. The entire method is geared toward validation of the uncovered phenomenon. The process begins with "naive perceptions" about the phenomenon which are then explained by outlining descriptors from the volunteers' stories. This concludes the investigation of the "human character of my object of research" (Van Kaam, 1966, pp. 261, 296).

In Van Kaam's (1962) research with the phenomenon of "really being understood" (p. 298), he shared that, initially, the object of the research cannot be confined and seen. Through review of the information, there develops a progression of discovery that continues until the experience can actually be labelled and its existence is perceived. For example, in Van Kaam's study relating to the meaning of being understood, one category that presented itself was the word "relief" (p. 307). The word allowed the phenomenon to be grasped. Accuracy is also achieved once the researcher recognizes the phenomenon through its description (Heidegger, 1962; Schutz, 1967; Spiegelberg, 1982).

\section{Generalizability}

Oiler (1982) noted that phenomenologic research cannot 
be generalized beyond the participants' involvement in a particular study, but that the data can be useful in broadening knowledge about an experience. This form of discovery can become the basis for other qualitative as well as quantitative research. Phenomena originate from human perceptions. For example, thought and reflection precede science; humans cannot live in their environment without arriving at conclusions that ultimately influence behavior. Phenomena are "things" and should be viewed as such. Ultimately these "things" become the data of sociologists (Durkheim, 1978, p. 14). Although phenomenological inquiry is not generalizable beyond the study participants, the data are useful in expanding the information and knowledge pertinent to other people in similar situations. Oiler (1982) wrote, "People live forward, they know what it is they have lived through only by looking back on it" (p. 179). No two individuals live an experience in precisely the same manner. However, common qualities can be identified from the subjects' stories (Heidegger, 1962; Merleau-Ponty, 1964; Schutz, 1967).

\section{Building Awareness of the Phenomenon through Language}

Building awareness of the experience occurs through either written accounts of or interviews with people selected because they have Iived through the experience being investigated. Language becomes the process of 
understanding the "moments of the experience" (Van Kaam, p. 301). Language renders the phenomenon explicable. Husserl stated that language explains the phenomenon, while MerleauPonty (1964) believed that language was part of the phenomenon. Heidegger (1962) considered it as the structure within which being exists. Language is the explanatory domain of experience. "The ego thinks, perceives, wonders about something: Ego-cogito-cogitatum" (Scudder \& Mickanus, 1985, p. 93). The words document the essence of the event and tell the story that has been perceived and lived.

\section{Ethical Considerations}

The strategies employed to ensure the protection of confidentiality throughout and following the study included the coding and matching of the participant's taped interviews, demographic data and transcriptions. As a result, no link could be established between the nurses and their stories other than by the researcher. All information, including the signed consent forms, demographic information, tapes and the transcription of these.tapes, was filed in a locked drawer until the project was concluded. To preclude the transcriptionist from being able to identify names and information from the tapes, code numbers were assigned to each participant at the time of the interview. A note book containing the assigned codes was also kept in the locked drawer in the researcher's home. 
The study proposal was prepared and reviewed by the University of San Diego's Committee on Protection. of Human Subjects. After approval was granted, selection of study participants was begun. Once contacts were made with each volunteer, the research topic, its purpose, the time of involvement and reimbursement were explained. Each volunteer was informed that the interviews were to be taperecorded and that, in order to participate, each participant was asked to sign a consent form (Appendix B). If they decided to withdraw at any time following the initial interview, the participants would still receive the stated amount of money for their involvement. The participants were also informed that further contacts might be necessary to clarify information. At the time of the initial interview, all questions regarding the study were answered. Following these interviews, all data were maintained in a secured area so that no one except the researcher had access to the information. The participants were also informed that at the conclusion of the study, all taped interviews would be destroyed and that they would receive a summary statement about the findings.

\section{Summary}

Van Kaam's methodology was implemented in this study and the process was discussed in terms of data collection, categorization and analysis. The role of the panel of 
experts was identified as well. In addition to a description of the participants, criteria for inclusion and the mode of participant selection were clarified. Maintaining trustworthiness, rigor, dependability, confirmability and accuracy were also outlined. The twelve step process of the phenomenologic inquiry was identified. A brief explanation of the role of language in phenomenology was presented. An explanation of the ethical considerations of the study was outlined in terms of the protection of the volunteers' anonymity and maintenance of all the data. 


\section{Chapter Four}

\section{PRESENTATION OF THE FINAL CONSTITUENTS}

Chapter Four will be a presentation of the final constituents of the phenomenon that was the subject of this study. The perceptions were labelled as Level I constituents and include becoming aware of the clients' ethnicities, drawing conclusions about these ethnicities through past experiences, making assumptions about clients and finally taking note of challenges, such as, language and reciprocal understanding.

Feelings were organized as Level II constituents and will be discussed as feelings of heightened awareness, empathy and compassion, feeling accepted and trusted, feeling a sense of triumph, rewarded and satisfied, feeling challenged, inadequate, frustrated and angry, worried and threatened, pressured by time constraints, resentment, and discriminated against by clients.

The Text

For this study, the question of import was "What are the lived experiences of nurses' interactions with clients 
who are ethnically different than themselves?". In order to uncover the constituents of this phenomenon, fifteen nurses were interviewed and their conversations were tape recorded. Each tape was professionally transcribed. Following the review of each transcription, all data relating to the phenomenon were listed, categorized and reviewed by a panel of experts. The final constituents were labelled. These final constituents will be presented and supported with the participants' statements throughout this Chapter.

Level I Perceptions (Object Directed): Becoming Aware of the Client's Ethnicity

The most common methods that nurses implemented to determine the client's ethnicity were through observations of physical characteristics and body adornments, dress, language, English fluency/nonfluency or the speaking of some other native language. The most common native language was Spanish. other clues utilized were observations of beliefs and practices and presumptive clues such as, address, legal/illegal status or other criteria known to the nurses.

\section{Physical Characteristics, Body Adornment and Dress}

Skin color was only referred to in shades of "dark", "darker" or "Iighter". The following are statements made by the participants that identify the characteristics that gave them clues about ethnicity: 
Well I think you use all of your sensors to decide that. Obviously they look a little different than my ethnic group. Their skin is darker, their voices are different, their actions are different. I mean everything seemed different to me and you could tell that right away.

It was only her skin color that clued me in that she was not probably Caucasian American.

His features were Asian.

She looked European, you know, which is probably a broad speculation...

Just her overall dress and stuff.

I would have thought he was chinese of some kind, if they hadn't told me he was Korean.

Well I guess I am not sure if she looked Spanish I mean she looked Anglo Spanish. So when I kinda looked toward her husband, he said, "oh she just got back from Turkey. That is where we are from."

They looked Asian but they were wearing American clothes.

Well number one her appearance. Well dark skin, dark hair it was obvious, I thought she might be Hispanic but I believe that she was probably an Indian.

This lady was Caucasian but I don't know what background she could be, Hungarian or anything, but she looked Caucasian to me, a very nice lady.

He had Asian features. It wasn't Filipino because there were some Filipino RNs and they couldn't communicate with him either.

One Anglo participant believed that she could differentiate Ethiopians from other Black patients. This is her conclusion:

They are pretty tall people and they are very dark. And they have a certain expression in their face. It was in their eyes and the way how they look. They look like they are not Africans, they 
are Ethiopians and they have finer features and they have different hair. Their hair is usually like they have it in Europe although it is still black. It is medium hair and they wear different clothes too more hanging on the body, some wraps. The men look also pretty much the same. They seem to wear the same clothes as we do depending on their financial situation. Then you just ask them right away and then they tell me (their ethnicity).

\section{Language: Lack of English Fluency or Speaking another}

Language

Becoming aware of clients' ethnicities was also based on the inability of patients to speak English, or speaking English with an accent unfamiliar to the nurse. Others were overheard speaking another language. These individuals were assessed as being ethnically "different" than the nurse in the following circumstances:

Well I knew as soon as I met them that they were Hispanic. You could tell by the accent and I could tell by the way the mother wore her clothing. I just know when I saw her that she was Hispanic there was no question.

Well I knew that they were Spanish speaking...

It makes you glad when you have a Spanish speaking patient that you may not be perfect in communicating but boy it beats what you had ...he spoke Spanish...he spoke only Spanish... They speak the native Navajo at all times.

I didn't understand word one of what he was saying, but he sounded coherent and it was another language. That is the oddest thing I asked around and no one seemed to know and in his chart, under other language, they didn't say: it was blank so I didn't know. 
Not often do we get couples that come in at the same time unless they have a language difficulty or for some unknown reason. And when I very first went in and I said, you know, introduced myself and said what seems to be the problem the husband answered for the wife and so finally I looked at the wife like, you know, why aren't you talking and she started talking in a foreign language and so I said oh what language? I am thinking maybe she was Spanish well she was from Turkey. He would interpret what I was saying to her in Turkish or his language and she seemed to understand most of what I said in English but would always come back in her native language and I was thinking - this is going to take forever, what is going on?

She was very well dressed, full makeup, hairdo, the whole thing you know obviously upper echelon of the Mexican society. So the problems should I go ahead and give the problem? She spoke only Spanish.

In her role as a Labor and Delivery Nurse, one nurse interacts with many ethnic groups. As her story unfolded, she mentioned that in some ethnic groups, the pregnant women are not married but:

Ethiopians mostly, yeah, those should be married because we can not communicate as well and we can't find out if they are really married.

Her assumption was primarily based on the difficulty that the patient had in communicating the information to her. She assessed her patient as speaking "Ethiopian" which she decided was the language of the people who "looked a certain way".

\section{Beliefs and Practices}

Beliefs and practices were also instrumental in alerting nurses about differences in ethnic membership 
between the nurse and the patient. For some nurses, knowledge about a person's beliefs or the observations of practices provided information about the ethnicity of their clients:

They took this baby to Mexico to a Curandera, who said the baby had a couple of folk diseases and one of them was caida mollera, fallen fontanel, and also empacho which was an intestinal disorder where there was a peach pit in the stomach or something. She said the curandera had pushed on the baby's hard pallet, pushed it up, and that caused the soft spot, the fontanel, to raise back up again. And that to treat the empacho that she used an oil, a warm oil, that she vigorously massaged the baby's abdomen and the next day the baby passed something that the mother described as looking like a peach pit. Well, and the baby began to improve, the physical condition of the baby began to improve. It seemed like that would have been about the 72 hours when you would have expected the antibiotic to really take hold. So it all worked out.

Alot of the population are migrant workers and they don't get medical care for their kids. They live out in the fields and work in the fields and that is where they raise their kids. And so the one that comes to mind most is a family that came in with a child who had been sick for a long time, was not eating well, was vomiting, had a huge abdomen and they didn't know what was going on.

And they (Navajos) usually are barefoot. They live in Hogans. I love their association with nature, the idea that this land was given to us and we must preserve it and love it for what it is. That there is a reason for all the little creatures in the world and there is a reason for all the different plants and flowers that we have and they are not ours to destroy so lets protect them. And this is the very basic belief of the Indian cultures of our land. They don't consider owning property. What they own are there personal belongings. They are kind of nomadic in nature but they are beautiful people. 
This particular family also had a burning candle and nothing in the house except the refrigerator and then had this candle burning on the fireplace. It was like a religious candle and I said, "you know with all these kids in the house," I mean the apartment was not that big and you know how kids jump and run and push, and shove and I said, "you know this really is dangerous" and she said. "no one ever touches it" and that was that. I wanted to blow the thing out and toss it but... that was one topic she was not going to budge. I still felt that at least I told her and I pointed out the problem so somewhere down the line maybe she would use that information or at least she could never say, "well nobody ever told me". So I felt it was my obligation to at least point it out.

In the following incident, the nurse was telling the story about a young woman who had been referred because of a "liver tumor" when, in fact, the diagnosis was pregnancy:

The part that struck me number one was that she had been to a doctor and this doctor had never examined the lady because in our culture (Mexican) you know normally if you are virginal or claim to be virginal, doctors will not do a pelvic exam on you.

This same nurse said that in the case when the person became pregnant out of wedlock, they were sent away. The destination was dependent on the family's financial situation. They go:

Even to Europe, you know. People used to take a little visit you know, when in reality you were pregnant and you had your baby over there and whatever you did with your baby, adoption or whatever, that was up to the person I guess.

As a (Mexican American) nurse practitioner, this volunteer noticed an interesting belief about one ethnic group:

I have a lot of Filipino patients and I am sure it is not just them but it just sticks in my mind that if you don't give them an antibiotic for 
their cold you are not doing your job. So it is frustrating for me trying to convince them that an antibiotic is not going to cure your cold.

Determining ethnicity through another practice allowed this

Mexican American nurse to decide that this man was a member

of an Asian ethnic group:

Well when we were giving him his bath, we had to untie his restraints and we sat him up. He was a little weak but he was able to sit up fine after a little bit. And he started to cry and the first thing he did was that he put his hands together and he bowed at us. One at a time very slowly and he kept crying so in my ignorance all I could do was stand back and bow at him also. And that is when I knew he was different.

When asked if bowing helped her make the decision about this man's ethnicity, this same volunteer said:

Yes, yes, in some Asian cultures they do that, either as a sign of respect or hello or whether he was praying at the time because he had his hands together, I don't know because that was another gesture that will give it away.

There were other practices that this nurse was aware of that helped her determine ethnicity:

Oh in some cultures too, the Asian, Filipino and so on, there is a hot/cold theory in play it seems and that is something I am familiar with in my own culture so when I have a patient who doesn't communicate with me then I know the water pitcher that is by their side, they are not going to want ice in it. I know every now and then I should perhaps bring them tea. Because I want them to drink something because I have seen family members who bring in different types of tea. So I try to have that.

One Anglo pediatric nurse was recounting the experience she had with a child whose parents had exercised a Southeast Asian practice: 
It is common that when there is an illness that they try to release an illness through these different methods it is like a medicine man type or, you know, care and they do actually burn them as to provide some release from this illness and then the coin rolling. I can't remember exactly what that means but they do that on the chest and on the forehead and from there again releasing whatever is causing the illness, the demons or whatever.

\section{Presumptive Clues: Address, Legal Status, Surname and}

Intuition

Some participants shared that they had the ability to determine the ethnicity of a patient by their address, legal or illegal status, surname or "intuition". When they verbalized this information, they seemed to feel comfortable with their decisions. For those nurses familiar with San Diego County, the client's address served as a clue to their ethnicity:

Her address, and I think her name. Am I allowed to tell you where she lived?

When the researcher gave her (Anglo nurse) permission, she continued:

Pauma Valley. Well, that is an area that is heavily populated by American Indians.

This particular nurse felt comfortable assessing a client's ethnicity by the address or assumed address. When she was asked if this determination was an assumption, she corrected the researcher and said, "an intuition". Another volunteer said that for her, addresses were clues to people's ethnicity and class: 
Well we could tell even if they were street people but they made up an address. We could tell that they really did not live there. Because they made up a fictitious address and when we picked up the care we could tell from their addresses frequently if it was a made up address. Many of them could not even write. Because we were so close to the border I think perhaps we were more suspicious than we should have been of this group of people (Mexicans). The Spanish, Mexican clientele, we felt that if they did not learn English they were probably illegal and that was probably a wrong assumption. Because perhaps they did not have time yet to learn English. Perhaps they did not have the inclination to learn it.

One nurse (Anglo) who was with the Public Health Department was very familiar with the geographic area to which she was assigned, therefore, she felt certain about determining ethnicity by a person's name and address:

Well how about the name? The name was a giveaway I would say. Well I knew from the area that they lived in was predominantly Hispanic and of course I knew when I saw the mother that the family was Hispanic. And the living conditions of course were, I had never seen anybody live like that. So I knew they were Hispanic. So what it involved was this little three year old who as the story went had contracted $\mathrm{TB}$ when he was with his grandmother, of course way down in the interior of Mexico and the mother had lots and lots of kids and had a husband who was an illegal so he came up when ever he could. And they really had no money and it was difficult because this child needed medication desperately.

After being asked if one participant (Anglo) considered the whole picture in determining ethnicity, she added:

Sure because there are some people with a Hispanic surname who are not what I would call culturally Hispanic even though they do have that name. So that is really not a good indication either. You can't just go by the name.

Another nurse (West Indian) stated that the name wasn't 
the only determinant. The accent was another clue:

By her name. I think she was English speaking as I recall but maybe had some accent which would identify her further. She was from Texas.

Another (Mexican American) was trying to decide the ethnicity of a gentleman who was Asian, at least according to her. No where was there any mention of his ethnicity and the family was not available to assist, so her approach was:

Just somewhere in my mind, there is a list. He looks Asian. I am not sure which particular ethnicity but it has to be an Asian ethnicity and it's not Filipino and it is not Japanese because he doesn't look too Japanese. His eyes weren't as narrow and he just didn't look Japanese and it has gotta be something else maybe Korean and ...

\section{Drawing Conclusions About Clients' Ethnicities}

According to Maslow (1970), people draw conclusions about others depending on a variety of criteria. He referred to this process as rubricizing or forming rubrics. Lippmann (1922) referred to this as forming "pictures in our heads" and Fishbein (1967) called this activity stereotyping.

A rubric is a descriptive statement that either identifies a whole group based on some observed attributes of individuals or attributes assigned to a single person based on his/her group membership. Examples might be presented in the following statements:

\footnotetext{
"They (a group) are usually all on welfare."

"She must not be literate because she is (an ethnicity) and they (a group) never attend school."
} 
As the interviews progressed, it became apparent that many of the participants arrived at some conclusions about their clients based on various attributes that seemed to be representative of the entire group to which these patients belonged. Of interest, was the fact that this information was not solicited by the researcher but seemed to emerge naturally from the nurses' experiences.

Role of past experiences: Several ethnicities were discussed by the participants, for example, "Asians", "Blacks", "Caucasian/American", "Ethiopian", "Hispanic", "Latinos", "Mexican", "Middle Easterners", and "Navajos". In recalling their experiences, the nurses told of their version or ideas about the ethnicity in general terms, in stereotypes or categories. The use of the collective word "they" was frequently verbalized. These categories were based on the nurses' past experiences.

For one Mexican American nurse, trying to determine her patient's ethnicity became a time consuming challenge. Although the medical record did include the patient's ethnicity, no documentation was made about an inability to speak English. No family members ever came to assist him. She explained the mental process she went through:

Well just a whole different scenario of different Asian cultures that he might pertain to... Perhaps he's from Korea and he spoke Korean because it didn't sound quite like Japanese and it wasn't Filipino so the other option I had was Korean. But I know that there are different 
dialects in the Filipino culture and he certainly could have been another.

Another Mexican American participant who was more comfortable with Spanish speaking clients was assigned to a male patient. The referral stated he could not speak English. She shared that this assumption was made by others and it caused her much anxiety prior to her visit to the man's home:

Well I got a referral at public health for a patient, a Korean patient, who had active tuberculosis but it wasn't only active, it was the kind of tuberculosis that, you know, he was sensitized to all the medications which means that he was very difficult to treat. And so they had already tried all kinds of treatments and he wasn't working with them. At the referral, they also said that he didn't speak English and you know we didn't have a Korean translator. I thought, oh my goodness, what am I going to do with this, you know, and so then when I saw the address, I said oh no. Then to top it all, he is in this building where all these drug addicts hang out and it is on the last floor where I have to go up those stairs with my back and I had to go every day there and see all these druggies. I felt all these things saying how in the world am I going to communicate with him and how am I going to get him to get this treatment and I was suppose to give him an injection every day and I thought this is going to be something else. Then I researched it, where I could get a translator. We have translators you know and it was just awful, it was just awful. Thinking about it, but then I said, got to do it, got to do it. But what else, that is why they pay me right? No way out, so oh my goodness, I got my bag and I go all the way up the stairs because the elevators weren't working. To top it off, he didn't have a phone... So I just showed up at his door and knocked at his door and I thought, well we will see what happens and when he opened the door, you know, I introduced myself and I said I was a public health nurse and they have sent me because he was sick and I almost fell, he answered in English he said, "oh welcome, come in". He spoke some English. 
As this nurse retold this experience, she became excited, with hand gestures and a smile, just remembering how she felt when the patient spoke those few English words. She shared how relieved she was at that moment. An Anglo ambulatory care nurse recalled her experience with Filipino patients in this way:

I think I see it more in the Filipinos who come to this county. All of them are immigrants and many, the older people you know, I get along alright with the older people. I don't have a problem with the older people it is the young kids who obviously have not really made any attempt to assimilate themselves into our culture here.

When this same nurse was asked how this affected her practice, her comment was:

This is one ethnicity of no discipline for children. The kids are told the doctors are mean, the nurses are mean and the kids scream the whole time they are in there. You know these things are very difficult to deal with. It is an opportunity to teach if you could hear them say it but you don't often hear them say it. You know very well that these children are threatened by a shot. It is like the doctor is a boogey man. And I think that this is very, very evident among the Asians.

Some of her other concerns, based on previously lived experiences were:

The other is just overcoming the cultural fears that they have. The Asian cultures, they don't understand the need for psychiatric or mental health care. They don't understand things like alcoholism, what is it that they need to do and that there is help for them and that there are Spanish speaking ALANON, AA groups in San Diego County. Getting it through to them that there is help for them, that they can find relief from these intolerable burdens that they think they are carrying.

For one Anglo volunteer, caring for some African Americans 
("Spoiled Blacks") was problematic:

Whenever they come through the door, they have this attitude, you're here to jump and you are here to do everything for me and give me first class treatment and now.

She was asked, how she knew this:

Well they come in and they want to be seen right away and they want a private room. And they want, right away all their medicines. There is a soda machine, "can I have something to drink," and I mean this is not just patients this is visitors alike and the visitors, you can tell them twenty times, please, it is too busy this time. Nothing, they come with a whole entourage.

This same nurse was asked how this situation occurs.

She shared her values about African American women in these words :

Most of them are raised into all female homes. There is no real family and alot of mothers are on welfare, grandmothers on welfare, great grandmothers on welfare, and they are born into this situation and they are really raised in that situation and often, going through the same footsteps and that is true with any kind of ethnic group. I think people if they take advantage of you, if they come in and do take advantage, mainly, I think they do this, my assumption is they do this because of insecurity, not very intelligent, you know, they haven't learned how to behave in a different situation..."

A Chinese American nurse recounted her experience with a mother who delivered and whose baby died shortly after birth. Her account was that this lady did not come to the hospital when she was told and had elected to wait several hours before delivery. The patient arrived with a ruptured placenta and the health care team, in her opinion, had done everything possible to save the baby. Several days after 
the incident, the patient sent thank you cards to each staff member. Within a few weeks following these cards, the nursing staff received notices that they had been named in this patient's malpractice lawsuit against them and the hospital. When asked how she felt, she recalled the following, including her conclusions about how this situation occurred:

I was terrified that I would lose my license, you know, even knowing that I have not done anything wrong. I think because she was born and raised in this country (America) had a lot to do with it. Because the way our legal system works and people are very sue happy and..."

She was asked what did she think the reason was for the lawsuit following their receipt of the thank you cards:

I think that at that time she was so involved in her loss and all that and that as she had time to remember that I was her nurse. She may have sought it out herself to seek legal help. Society like her in America, the people are very sue happy.

She had received her nursing education in England where, as she said, malpractice suits are uncommon:

Some are very legal cultures. This is a very legal conscious country, I think, and the lawyers will sometimes approach patients and if they find a case they will approach the patients.

Conclusions about members of the Latino group were described by utilizing interchangeable labels such as Mexican, Latinos or Hispanics. There were more comments made about these ethnicities because of their predominance in the experiences of the study participants living in san Diego County. 
An Anglo public health nurse shared what she had learned from one experience with a young Mexican child with tuberculosis:

Well it was a good learning experience because I learned that people from deep down in Mexico never admit to having TB even if they are sputum positive and skin test positive they never admit it. They never talk about it. They never want to tell anybody else they have to get a skin test. Well I don't know what it is but I know after my experience with this little guy that it wasn't uncommon and other Hispanic families were the same and I finally got to the end where I knew if they were Hispanic that it was going to be up to me to find the contacts and tell them that they needed to be skin tested because I knew that they never would.

Based on her personal feelings in caring for Mexican clients, one Anglo ambulatory care nurse shared:

I resent the fact that many of these Mexican people especially have lived in this country for years and have made no attempt to learn the language. I think they take advantage of our culture here and our medical care but have done nothing to help themselves. I feel in parenting many of them lack the basic parenting skills that they need.

In assessing the marital status of women in labor, one Anglo participant stated:

The funniest ones are really the Mexicans to take care of because with the man we know already that most women are not married and the man takes off as soon as the baby is there (born).

When asked how she came to know this information, she stated:

Well it is repetitive. We are told, "no, I am not married", and then the man is gone and I say to them, "aren't you just going to stay here and raise these kids?" or "were you just present during the production" and I can joke with them 
because I can speak their language and I say, "I want you to raise these kids. Here, its yours too and I get usually a very funny response.

In terms of the female Hispanic patients, this same nurse added:

Usually they take fairly good care of themselves. They are not very demanding and you feel like you can make something good for them. You can give them a wonderful day and even though they are in labor and have pain, you can really treat them such that might be the best day in the rest of their lives.

For another nurse who treated a ciient with tuberculosis, she recounted how, during one visit, he had a "psychotic reaction" to the medication she administered to him. In attempting to describe her assessment of this experience working with a member of the Mexican group, she said:

Well I feel that probably anybody here illegally is already frightened. So they already are susceptible to this (reaction). They sneaked across the border in order to get the treatment. And then they don't understand the treatment they are getting. They don't know and I never tell people that (about possible reactions) because it is so rare.

When asked to explain more about her experience so all could become more knowledgeable about Mexican people living here, she continued:

Well to understand that they are lost here frequently and they don't feel comfortable. Therefore, instead of making fun of them and criticizing them, we should try to understand and help them become Americans. Also that is what they want to be.

Another Anglo participant drew the following rubric about 
Mexican clients:

They come in with lots of people, friends, whoever helps them out, whoever brings them in, sometimes I am surprised, I mean, the brother or the brother-in-law. I say, "I wouldn't like to have my brother in the labor room. Why don't we close the door, send him out, and she says, okay".

For one nurse practitioner, the assumption about a well dressed young Mexican woman made her diagnosis more challenging when trying to explain that the patient did not have a liver tumor but was eight to nine months pregnant:

okay so here is the obviously nice, well dressed lady. I am assuming that she is educated and I am assuming that she knew the birds and the bees. Well, I mean about the facts of life, about how you get pregnant and I guess she didn't or at least not fully anyway and I guess she didn't understand that it is possible to get pregnant without having actual penetration. So I am assuming that she knew this thing but obviously she didn't and there was the proof, a pregnant belly and fetal heart tones and all that. Again, I come from a humble background. She comes from a more affluent background, my assumption was this lady is educated you know she would know what is going on and obviously she didn't and so that is a little bit different and then that was an assumption on my part.

For one nurse, past experience allowed her to conclude the following:

More and more Hispanic people, they are demanding so much. Our number one thing is, "do you speak English or speak Spanish?" There is one incident that happened to me several years ago. A patient didn't want anything to do with me because I'm not a Spanish speaking nurse. A doctor had to speak up for me. He went in the room and said, "look here, you know you are in America and that is what you should do, speak English".

One Anglo pediatric nurse practitioner had drawn conclusions based on her experiences with members of the Mexican 
American ethnicity:

It seems to me that through experiences with previous mothers, that there were certain pitfalls that you could expect these mothers to fall into. like my experience and I may be all wrong, is that Mexican mothers tend to be more permissive with their children than, American mothers. That they are more likely to put babies to bed with bottles and so you always want to be sure to bring these issues up with how dangerous it can be to put a baby to bed with a bottle and those things. I have this feeling that they are very good mothers. This mother always called her little girl

Mamasita. It is like you have been brought up to be a mommy. I am not raising you to be a rocket scientist, I am raising you to be a momma (mamasita).

While describing her experiences with patients from the

Middle East, one nurse concluded the following:

Those are usually the educated ones. The women from the Middle East are very needy. They don't take charge of themselves. They are kinda just, "here I am, take care of me" and when they are in labor they want to be taken care of. They want to be rolled over, they want to be looked at and they become very insufficient in self care. They want us to take care of them. They want us to take all the burden away and do it for them and you can not do that.

When asked how she personally drew these conclusions, her response was based on:

Just total facial expressions and the way how they carry their body, you know, the gait is totally collapsed. You get a feeling for it.

A Nurse Practitioner had not told a doctor what language a patient spoke. The following was the result:

When I told him that she spoke a different language he automatically assumed it was Spanish since most of our clients are Spanish speaking. And he is Spanish. And maybe he thought she looked Spanish too so he comes in and starts talking to her in Spanish and both the husband and 
wife just smile and look at him like we don't know what you're saying. I said, "she is from Turkey." He said, "oh oh oh oh oh". So anyway, he told him (the husband) what we were going to do. He took out the IUD very nicely, very smoothly. She didn't have hardly any discomfort what so ever and I decided to work her up for not only tuberculosis but put her on an antibiotic that would help not only with her pelvic inflammatory disease but hopefully with her bronchitis as well.

In San Diego County, the areas of Pala and Pauma are reservations where several Native American tribes reside. One nurse told of her experience in caring for a young, pregnant, Native American teenager who appeared to be very frightened when she was admitted in false labor. After the admission, two male members of the patient's family created a scene at the nurses station. Her conclusion was based on past encounters with members of this particular group:

They drink too much and they come in with friends and they can be very threatening plus the fact that what is happening in this day and age, it was very threatening to me, I mean his behavior. I mean, I wasn't worried about the lawyers and the reporter. That, I would have been able to handle but it was the possibility of physical violence that worried me, against the nurse plus all the other nurses on the unit. We do have several patients that we do get down from Pauma Valley and I find alot of them are more, I don't want to say defensive, they seem to have an attitude.

\section{Making Assumptions about Clients}

As Lippmann (1922) explained, people do make assumptions about others based on certain attributes. These assumptions become mental images that are difficult to erase.

Two of the nurses in this study chose to be 
philosophical about assumptions which they were aware of and mentioned in their stories. They were candid about the potential consequences of their assumptions. When questioned about personal beliefs based on assumptions, one nurse shared:

I guess it has to do with your upbringing, your culture, education or lack of and you know you just take the $x$ number of facts and you assume that $x$ equals $y$ and that kind of thing. I guess that is what the psychologists and psychiatrists are still trying to figure out. We shouldn't assume anything ever but you know we do, we are human. You forget that or at least momentarily. It is not all the time, sometimes. People are different and that you shouldn't make assumptions but we still do. I do it even though I know I shouldn't do it. Again just don't take anything for granted; everybody is an individual. It doesn't matter whether it is my same culture or any other culture. You still need to treat that person as a person, as a whole don't make any, again here we go to that word, don't make any assumptions basically just that. We make all these generalizations and assumptions but you never know, it doesn't matter walk of life you come from, if you don't know, you don't know. If somebody didn't teach you it doesn't matter again where you come from, you can still get caught not just in that but in a lot of other health issues to. The challenge is not to be ethnocentric. I guess that is the right word but that is what I want to say. You know what you believe and hopefully you are not imposing your beliefs on everybody else. And so just basically be aware that people come from different backgrounds and to be sensitive to that. That is all I can say in summary. Again don't assume anything.

Making a decision about a patient's ethnicity based on an assumption, resulted in one participant's decision that a mother was Hispanic when in fact she was a Pacific Islander. The nurse volunteered this advice:

I think that is one of the things that I came away 
from that particular interaction with the feeling that don't assume anything. Don't assume because the people look Asian that they are for instance or because they are a little darker skinned that they are particularly Mexican because we have a lot of Middle and Central American people and South American people that are Spanish speaking but we treat them all as if they were Mexicans and they are not and they don't have the same ethnic kinds of beliefs. Our Mexican families have some things that we are used to, some of their folk medicines and things that we are familiar with. We could, well, be incorrect and it could be I think insulting just to think that somebody belongs to a particular group and they don't at all.

She added that one "pitfall" resulting from assumptions is:

They can put you on the defensive because well they can affect you or it can affect your patient because you could turn them (the patients) off and make them negative towards you by assuming that they were something that they are not and it can also make you defensive because you have been caught.

\section{Taking Note of Challenges}

Many of the participants mentioned that they had felt challenged by certain aspects of the client's ethnicity. The language dissimilarities between the two of them afforded opportunities for the nurse to become creative in attempting to understand the patient and be understood as well.

Ianguage as a barrier: For this study, the major challenge was for the nurses to "reinvent" nursing care measures when the client did not speak English or when the nurses' use of the client's language was limited. As each participant 
shared lived experiences, the barrier was identified as well as the method used to meet the challenges of:

1. "understanding the patients' needs"

2. "being understood"

3. "showing caring behaviors"

4. "educating through "mimes" and "show and tell"

Reciprocal understanding: When a common language was not spoken by both the nurse and the client, the more difficult it was for the former to perceive whether or not reciprocal understanding had taken place. The participants stated that in these situations, they became dependent upon their physical assessment skills and their ability to observe facial cues as well as nonverbal language. Nonverbal behaviors were perceived as a "whole different ball game". When participants were asked how they managed to understand what their clients needed and how they themselves attempted to be understood, some comments were: "looking for "grimacing" and "guarding" as signs of pain, "smiling" as a clue to understanding by the patient, "reading their faces" for signs of depression or sadness ie. "mouth sags", "eyes don't sparkle". When utilizing an interpreter, nurses would look toward the patient to observe appropriate responses (facial expressions) and to denote understanding or proper translation. One nurse said that she believed that some nonverbal expressions (smiling, crying) were part of a 
universal language. When asked how she came to know this, her response was:

It is placing my values on facial expressions and so on them. But I think it is something that is cross cultural. I believe because I seem to have been correct in most instances just those things, because in their languages, their tone varies and something that might sound happy is really sad but their facial expressions I think is something you can't change. I think it cuts across all cultures, you know, smiling is smiling and crying and mourning is, you know, being sad. It seems to me that it is something that goes, cuts across.

When asked how she learned this, her response was:

Well we did have some classes in cross cultural beliefs and so on but it was kinda funny too, I know that in National Geographic when I have read or looked through it and you look at different tribes and stuff you see when they are doing different native dances they have expressions on their faces and you don't know how long National Geographic has been there with them training them to be Americanized but it just seems through different things like that and through television.

For many of the participants, having faith in physical assessment skills and watching for facial expressions and body messages were what allowed them to have some inclinations about the patient's needs. The next stories will illustrate how nurses understand about patients' "needs" and assess whether or not they are understood by these clients. One Filipino nurse cared for a Hmong woman in labor:

Well I think language was a large part of it. I think that was really the biggest barrier and again out of this experience you know they seemed to really appreciate what ever you did for them even though you did alot of sign language. Well if she was getting more active, if she was grimacing or if she had more perspiration, just 
see her body appearance. How labor was progressing so then you, as well as you could, helped her and that is what she needed. Whenever you assess that you know that the patient doesn't really understand or actually there are no relatives that can speak English and throughout a twenty four hour period, we go back again and try to figure out just by looking at the patient, assessing expressions, facial grimaces. Alot of that and just try to correlate that with how their labor patterns are going. I think in that

situation when I can't communicate, I usually stay with the patient and let them know that you are there. You have to be there. You can't communicate. I stay with the patient and just do some of the comfort measures. I think one of the things was not touching the head or if they were sweating just to kinda wipe them off, that kind of thing or just in a soothing way even though she doesn't understand. Yes and just saying, "okay, are you okay?", making sure I don't tap the head or whatever and she would say yes.

The next experience occurred in a hospital setting when one participant cared for a Navajo child who was in critical condition and her mother could not speak English:

The nonverbal communication is a whole different ball game. There is alot of nonverbal communication in nursing if nurses choose to look for it. I think maybe this is the value of my own particular training is that we were taught to look. During the day, that day, we talked sign language. We comforted the mother the best we could. We fed her, we gave her towels and things so she could take a shower and clean herself up. We did all these things for her and she smiled in return but she was unable to speak to us. Indian language is a very, very difficult language. The sounds are very difficult to understand. Well we allowed the mother to help when she could. This is very important. She wasn't told she can't touch the baby. And I think this also eased her mind. You know, you need the sense of touch. Children need touch and we let her help when she could with the child. It was very painful for her (the child) to move and to keep her moving just simply to dress the burns. We had to. These things are very difficult. She needed her mother there to talk to her, to explain to her what we 
were doing. The fact that we did all we could for this child and maybe the next time we would do better and that we have supported this mother and that she was grateful for what we had done. You know there are expressions in gratitude, usually a smile and they know that you tried.

An Anglo nurse verbalized that understanding occurred through her actions while caring for a Native American child:

Oh my goodness I just wished I could reach through to this lady and tell her but by our actions, I think she understood that we were trying to help this child.

During a well baby visit, a nurse practitioner was assessing the mother's level of understanding about a basic feeding schedule in light of the mother's limited English fluency:

It was for a well baby exam. I don't remember whether it was for a six month exam. Of course the physical examination itself isn't a problem because you are going on your own information but when you try to get any developmental information or nutrition, what the baby has been eating and how often. And that was one of the things when I would ask her how much she was feeding the baby and how often because she was formula feeding the baby, I used visual cues alot. I watched the mother to see if she gave some indication about understanding what I was telling her and by repeating and asking the same question over when I didn't like the answer I was getting. I'd ask again until I got one that at least made sense. Like if I asked, "how often are you feeding this baby" and she would say "4 ozs", I obviously didn't like that answer so I would ask over again and kind of point to the clock or to my watch. Also, by speaking slowly and trying to get some verbal cues or body clues of some sort that she understood what I was saying. But I think that our communication and the whole process of my examining this baby and teaching the mother was really hampered by the fact that our communication was limited.

A baby was brought to the hospital for care. His family was Southeast Asian. The child had been treated at 
home with coin rolling and skin burnings. None of the family spoke English:

They were wearing American clothes. They looked Asian but we didn't know what was their ethnic background until a person told us and then he also did share that they were from the mountains and they also didn't speak very much English so you get bits and pieces and you are just hoping that they are understanding your questions and giving you the correct information back. What was interesting about this story is that they were very attentive to the child and stayed most of the time with him and held him and were very close to him and he was very well nourished. Growth and development met all the criteria for his age.

This particular participant was asked how she made herself understood:

Well you try very much to communicate with them. Alot of stuff is done by sign language kinda like mime. I mean like just showing them things and I think also when they come in they can see that the baby feels better, looks better, eats better. There is a lot of that kind of thing, you let them hold the child and they know if things are better. If they can't hold the child then they can see that he is sicker and he has to stay and he is connected to various things, IV's and different things. In a situation, if you come in and the family comes in and you are trying to talk to them anyway even though they don't really understand the English, you point and you just try to communicate with them with just different words and the tone of your voice because you know they aren't understanding the words.

one nurse shared how she attempts to determine understanding

from clients or their family members:

Well it seems like when you look at their response to what they have said if they become solemn and talk among themselves with a certain tone, you think, their facial expressions look concerned then you think that maybe you have conveyed it or you can kind of tell by the way people look whether they are confused or whether they are concerned. You just know it. You feel it. You 
feel that they feel comfortable with it or that they have expressed it. If there is an interpreter or just through facial expressions they seem to be pleased with what is happening and they feel comfortable with you. They try to communicate with you. That is another thing too, I think they are usually more hesitant in communicating with us than we are communicating with them.

Another challenge in being understood occurs when nurses

have to relay different messages through facial expressions:

Your facial expressions are very solemn or ... if you hand them the baby and they can carry the baby then they feel like things are better but if you keep the baby in the bed and like say, "no" they maybe will sense that the baby is not doing quite as well and then they will be looking at the baby and you can point out different things like you know if the baby is wearing oxygen or something, you just point.

When women are in labor and they do not share a common

language with their nurse, the following may result. The

nurse told of her experience with an Ethiopian woman:

It was in the context that the woman delivered pretty rapidly too. And I couldn't speak to her very much but I just would show it to her with my hands, a lot of gestures. I showed her how to breath and relax and before I gave her an IV I would just show her the things and where I would put it and then the fetal monitor. I would tell them, "baby", they could understand "baby" and I was telling them that was the heart. If you adjust the fetal monitor or you look to see if she broke the bag of water and then you just say, "please leave the sheet down". It is only me and you and your husband and then you just make sure the door is closed or the curtain is closed and make sure she has privacy. I give her something to drink and support her back and her stomach, you know, just make her comfortable. I just look to see if everyone is comfortable.

Another participant, stated that the inability to communicate verbally with patients was: 
Simple in one way and it is difficult in another. It is simple when you cannot talk a lot. It stays simple and you do alot of just nonverbal communication. You only can get by with very simple technique, the simpler the better, and it is very difficult if you have a problem then you cannot talk to them and then you have to do things and they don't understand and you cannot explain to them what is going on and their eyes get bigger and they look kinda fearful and then you just do eye to eye contact. You talk to them in a very reassuring way and you use your sign language and you smile and you touch them on the arm and you say, "things are going to be okay". We just really make sure the best you can that they know what you are going to do.

A participant was eloquent in the description of her

lived experience with an Asian man who was unable to speak

English. She also offered lessons that help her in other similar situations:

Yes, when I came into the room he looked so abandoned. It didn't feel right just to come in and look at things here and there and not acknowledge that there was a human being behind the IV, behind the restraints, behind the catheter, and I think it helped him that someone did look at him rather than at the catheter at the IV and at the restraints. Someone acknowledged that he was there. Well I might have learned a lot if his family had been there. I might have learned about a whole different culture. I might have walked away with a lot had I been able to communicate with him better, had the family been there that I could talk to but the family never came in. He was isolated. The curtain was drawn. He was tied up and I am sure, if I was in that circumstance, I wouldn't appreciate it. I would want to be somewhere else. I would want to do something else.

When asked how she understood what he needed, her reply was:

Well, when you don't know what the problem is you don't know how to fix it so you don't know what to do. It is a little human instinct. I mean you don't like to see people suffer and if there is anything you can do to bring them some comfort, 
you instinctively try to find that something. Well, I knew he needed to be around people, so I took him into the hall where he could see others. He smiled and he didn't pull at his foley and he was more calm. He seemed more calm afterwards and he stopped crying. No more tears.

What was her biggest challenge with this patient?

To find some level in which I could communicate with him. Well, beyond ethnicity and beyond the cultural world is human beings. We have basic needs, hunger, cold, just basic needs and beyond everything else we are all pretty much the same flesh and blood. Yes, on that level I can communicate with them. I have that experience in common with them. I am human. When I can't identify the differences I have to go for the parallels.

In summary, she added:

With this particular man, after the encounter, there is not a doubt in my mind that he was better when I left him. More comfortable or feeling better than he was before the encounter.

Level II Feelings: (Subject Directed)

How observers perceive others in an interaction stimulate feelings or emotions in each encounter. The sensory level can be described by the participants and becomes enmeshed in the experience as it is lived and recalled.

As Van Kaam (1966) wrote, "when one reflects on the character of human experience, one becomes increasingly impressed by its complexity...; it suggests an appearing and disappearing of perceptive needs, emotions which interact in various ways" (p. 299). When humans attempt to explain their experiences, the person "feels forced to use 
comparisons and metaphors borrowed from the world of sensible physical events and objects" (p. 307).

In presenting the feelings experienced by nurses in interactions with ethnically diverse clients, the participants used many metaphors or similes in an attempt at allowing the phenomenon to become describable. Identifying the feelings of these participants added the emotional context to the lived experiences.

For the purpose of explicating the most common emotions identified by the nurses, the feelings were categorized based on similarities of the expressions, such as, "compassion" and "empathy", "inadequacy" and "not measuring up". In order to explore these constituents, the researcher needed to presuppose that "...other people do understand what is basically meant when somebody says, "I am happy", "I am bored" or "I feel sad" (Van Kaam, 1966, p. 312). The researcher labeled the feelings as stated by the participants. The most frequently experienced feelings were: heightened awareness, empathy and compassion, accepted and trusted, sense of triumph, rewarded and satisfied, challenged, inadequate, helpless, frustrated and angry, pressured by time constraints and resentment. Two volunteers also described feelings of being discriminated against by clients. 
Feelings of Heightened Awareness, Empathy and Compassion

The nurses described the feelings of heightened awareness, empathy and compassion in several ways. For some, metaphors and similes were verbalized to assist in the descriptions of their experiences. These feelings will be expressed in the words of the nurse participants:

I think it is just that we should all be aware of the way we would feel if we were in their place and it gets down to do unto others as you would have them do unto you. I think that I am just a little more aware of their feelings, a little more aware of their fright perhaps.

It is being able to make them understand that you care, that we really want to do for them what is best for them and we want to teach them how to care for themselves.

I think she was frightened and as I said I don't know what the situation was at home and you feel sorry for her.

Some nurses tried to place themselves in the patient's position which gave rise to feelings of compassion and empathy:

That everybody is the same, has the same feelings, you know. I treat everybody the same way I would treat a Spanish speaking patient and no problem. Well I guess I think I just learned that the basics for any interaction is to see the person with compassion, try to help them through their feelings and respect them as a basic human being. In Spanish, there is a term, "Respeto".

I myself have a lot of compassion for people and when I see them I put myself in their shoes and I treat them the way I would like to be treated. It doesn't matter who it is, I always think this person has feelings and he needs alot of respect and $I$ am going to do the best I can.

I think it is most important for me to allow them to still be the person that they are and still to 
have some of their beliefs but also to maybe be accepting of what we are doing and use some of our medicine or whatever and so to get the best of both.

We all come from different backgrounds and we all bring our unique beliefs and everybody's different and everybody's an individual so deal with people as an individual. Find out what they believe in. Because even within my culture there are different beliefs. It depends on what part of the country you come from just like Americans. People in the South treat illness a little bit different than people from the North and that kind of a thing. Everybody's different so try to keep it in mind.

Because I am from a different culture, it makes me a little bit more aware and more sensitive in dealing with people.

Again, I would like to think that when you are in the other shoe, how does somebody perceive me? I like to perceive them as a person, as an

individual and treat everybody the same as those from different cultural backgrounds. You try to take those into consideration sometimes it is easy and sometimes it is hard. I don't know the main thing would be to just ask the patient for clarification. I guess maybe do you understand this or do you have questions or how can I help you or what ever just so that they understand where I am coming from and hopefully I will understand where they are coming from.

A participant categorized nurses as feeling motherly, especially when caring for children:

Well I think that all nurses have this mothering thing and that is why they are nurses. I think they do. I mean most nurses that I know have this thing and not that you are a better parent for the child. You become very, very attached to the children and you have to really constantly remind yourself that you are not the parent. And I think it might even be sometimes worse for some people who don't have children because they may be fulfilling that parenting thing. It is just really a part of them and it goes much deeper than just the physical, having cared for them. It is really something from inside that they have been attached to the patient and this child. 
It is all the little tiny things that you don't think make a difference. I mean they don't know the difference whether you are hanging $500 \mathrm{mg}$ of ancef or whether your pushing a drug that is critical to their child. They don't know that even if they could speak the language. Your not going to communicate that part of it to them anyway so what they care about is that you try and make the child and the family as comfortable as possible. That you look and act like you understand or you're empathetic with their situation. You know if it is a serious situation, you don't go in laughing and joking with a friend of yours in English when they are all crying and sitting there talking to each other in spanish. They would not understand that. So it is little things.

We provided a separate room for them to be with him during the last times and let them do the religious ceremonies.

I periodically and people I guess should do this no matter what job they are in, my treating these people like I would want to be treated or like I want my child to be treated and if I have a six year old patient and I have a six year old child, I kinda know what she would want, if she was in the hospital. To me, it's what nursing is all about. The technical stuff changes all the time and it can be taught, I think, to just about anybody. The extra is more difficult.

When you have a first person translation, you have everything at once. When I do the first person translation after a while they start to look at each other and they start to ignore me. So they communicate with each other. I'm just a language filter between them and they start talking to each other. They look into each other's eyes when they laugh, they laugh with each other. They don't laugh at me and then it all happens at the same time. All the communication happens at the same time.

Feeling Accepted and Trusted in Building Rapport

Other participants were able to describe how they felt accepted and trusted by the clients while attempting to 
build rapport, especially when language was a major

roadblock. The feelings are presented in the participants

words:

The family will share with a nurse because there again the nurse is there at the bedside caring for the child and they, you know, no matter who the person is, what they have done to their child, they still love their child and they sense that you care for their child and so then they may open up to the nurse alot more so alot of times the nurse becomes all the other people, social worker, and so on.

Well if they complete the medication, because it is a long time, sometimes it is nine months, sometimes it is a year. That is a long time and so when they complete that, that's really like a wonderful feeling.

Other people accept it very well, okay fine. It is like they take your word for it because hopefully they trust you as a health provider but somehow or other the Filipino's are a little bit more challenging, shall I say, in trying to convince them about certain things. I guess they want what they want.

You don't explain what the $x$-rays look like, what medicines you are giving or anything. They are just completely trusting that you are doing the right thing.

I think just in that we really tried to include the family even if they couldn't understand what we were saying, we were very glad when they came. We greeted them we made them very much part of us and welcomed them into our ICU culture, our little group. I think I felt like we communicated. I felt that somehow they knew that we were caring for them and that their child's life made a difference to us. I guess that is a good feeling to be able to do that.

I think he trusted me, he said, "oh she is a nice nurse" and then I was very careful on how I did it because I knew that he didn't want this so I took my time I said, "where does it hurt" and "I don't want to hurt you so you have to help me. You tell me where it hurts." 
They are happy with their care or they are happy with what they had. They thank you sometimes they hug you. They smile.

Well it was funny. The thing that made me feel trusted was when she came back two days later, she acted so happy, you know. She kept saying, "oh oh" and patting me and she was better and her husband kept saying how well she was sleeping and they both were smiling and happy, that it was so nice.

You love to build rapport. With this client, it probably took me about a month before he could trust me openly because we didn't start functioning right away like the next day, but there was a period of time when he started opening up more and more. When he started sharing his most intimate details of his wife being illegal, being afraid of her being deported. I knew.

At least I knew a few words. As a result of this though, we developed a little bit of communication. It is just a feeling.

That is another thing that he accepted me because I did not wear a mask. I just know because alot of other nurses would have put a mask on.

Well you are going to do every procedure, every med reeds to be given that would be given. If I'm English speaking and I have an English speaking child I might go in when I have five minutes and read a book to that child or something very simple like that would only take a few minutes but it might mean alot to the family and the child. If I have a Spanish speaking child, I might not be able to read a whole book to the child so I could get a coloring book and take it into the child's room and color for five minutes with the child. And that has nothing to do with nursing by any means but in the parents eyes, it builds trust and they know that you care about all the different aspects of the child. The procedures are done and the

things that they think are invasive and hurtful to the child. That you don't only go in when you are going to do something like stick a thermometer in their bottom or give a shot or start an IV. You are going to go in for other reasons as well.

I felt really good because this mother trusted me enough to reveal the treatment that she had sought 
and that she described it to me. And I felt

further enhanced by the fact that I had learned something of these diseases and treatments while I was in school. So I was not completely ignorant of those kinds of treatments. It was kind of interesting that the mother would trust me enough to tell me this. Yeah that I understood something about what kind of folk diseases and folk treatments and so by having the mother trust me enough to reveal this made me feel very good.

A very positive feeling and I knew she was not going to leave our health care system to go elsewhere because she did trust us and continuity is really important with mothers and babies.

Feeling a Sense of Triumph, Rewarded and Satisfied

Despite differences in ethnicity, beliefs, practices and language, the participants shared times when they felt a sense of triumph, rewarded and satisfied:

Well after the baby was born the husband would say, "thank you very much for all your help" and they feel good that somebody was there to help them along.

I felt like this is great! Well, I can't believe this worked out so well. And then, when I got a note from the doctor saying that it went really well, that was really nice because you don't get too many of those often enough.

I don't know, if I could use a word that she could understand, I'd feel good about that.

A simple thank you and a smile and acknowledgment that you did a good job. Thank you for helping me through it. That to me is a good reward. With the Hispanic population sometimes they don't speak English. The look on their face, it looks like they are grateful. Their smiling, you can always tell when someone is happy.

I have been in this business a long time and I just don't have to have chocolates and thank you cards. A smile, that's enough that is plenty. 
Well I think you feel a great sense of triumph if you feel like you have communicated or educated them. That is a big thing with nurses, teaching and helping people learn to take care of themselves and so I think you just really feel like you jumped over a huge hurdle if you feel like you got anything across to them and they appear to be happy about everything you are doing.

They said, "thank you" in English. They would smile. You can tell by their expressions and their voices that they were saying something kind about you and the other family members would look and smile and wave or what ever. They would be happy to see you.

I think it just feels good to get through a day where you know that you can't explain things as easily as you could to the next person but you somehow get settled and you get the point across and maybe you learn a few extra words that day and so that feels rewarding, that feels good. Well I guess that you could just summarize it into just saying that people no matter what ethnic group they are in, what language they speak, how they look, how they are etc., everybody wants the same thing. And I know I have had a good day when I feel like I've done everything for my patients that I would want done for my child or my loved one that was in the hospital.

For some, satisfaction was experienced when changes in the client were observed:

The fact that he stopped crying, and the fact that he stopped pulling out his foley.

I think you walk away wondering whether you did make an impact or not. I guess that from the condition the baby was in she was either learning something from the health care providers or she had enough prior knowledge that her baby looked good. And so you feel comfortable with what she has done so far.

\section{Eeeling Challenged}

For many of the volunteers, each patient who presented with a different ethnic make-up or language afforded a 
challenge. Perceiving each client as an individual, caring for the patients without fear, accepting what they

misunderstood and making the best of each situation were the primary challenges faced by the nurses in this study. Some of the challenges are recounted:

For me the major challenge was to try to understand my own feelings in reactions to this child and not get too attached. To cry when I felt free to cry when she died. I felt this was a good release. I felt free.

I think I am more tolerant and more aware that perhaps they are just as fearful as we are and that we have to try to have a little more patience and a little more kindness, and a little more understanding.

The biggest challenges with that ethnic group (Hispanic) are that they are very affectionate people, they are very huggy and it is okay to give them a hug and to let them know that you care about what is going on with them. They need to have their family support with them even though it may not always be comfortable to have all those people in the room at one time. I think they feel like they are not getting as good care as the next person or the next child who speaks English. So you kinda need to go the extra mile to let them know that yes they are getting as much of your attention as the next person is. You do care that their child is hungry or tired or they are tired or hungry or whatever. So it is an extra effort that you have to make for things to go smoothly.

It is what your goal becores. Plant this idea (in patient's mind) and maybe nothing will be done for a week maybe not for six months but somewhere along the line they are going to say, "oh yes I remember she did tell me that" and so what you need to do is just give them the information. Maybe they aren't ready for it right away.

What was a big challenge was just trying to be nonjudgemental toward them (Sikh) and to keep in mind that this is their child and we were trying to help them with their child and not to take the child to become ours. I think pediatric nurses in 
particular become the mothers.

Well you just keep working at it and trying different avenues until between the patient and the family and yourself, you plan your care. You feel like together you have reached the right avenue and it is an internal feeling. Part of it is that the patient is better and the family seems to be accepting of what you have done.

Well I feel that the greatest challenge was to learn to speak Spanish. We need to learn to be able to communicate with these people and if they can't learn English we have to learn Spanish. It is that simple.

I think that the challenge is after the baby is born. The challenging part is to try to see that the care for the mom, baby and for the rest of the family happens. Many of them don't remember everything and their very grateful when you do help the mom with all that baby care.

Well it takes a long time. You have to just keep at it, keep trying and you just keep reinventing ways of getting your information across.

The challenging moment was trying to convince her that she was pregnant even though she did not have any intercourse or penetration. That was the challenging part. How do I convince this person who claims not to have had sex? How do I convince her that she is pregnant?

Mainly I think one of the big problems that we have was the language. It was a real challenge to be able to talk to them in relation to childbirth.

Actually just not to be able to speak to them and let them know what is going on because I like for my patients to know what is going on and to prepare them ahead of time and in this case you could not prepare them ahead of time. You can just anticipate.

You have to kinda play dumb sometimes and say, "Well what do you think", "How much does he eat during the day" and "When do you feed him" and just like they are the experts on what ever you are asking them. Treat them as though they were the experts and then they tend to pass on more 
information or you really convey an interest in their activities and their culture. They are really very, very willing to share how they live. Part of the game, because I think sometimes you have to play that with doctors too because you know that what you want to do is right and their idea may not be. You don't think they are going to work quite as well as if you put your idea in their head and make them think it was their idea. you know what I mean? So you play those games with lots of people in your lives.

Well I think year by year I have gotten better at not being afraid to try, not being afraid to approach a family even though they speak a different language. Kids especially, all they want is someone to play with they don't really understand any of the other part anyway so it makes it a little bit easier to be working with kids all the time. And not to feel inferior or less of a nurse because I can't speak that language fluently.

I have noticed the harder you try with every different ethnic group even if I fumble through every word, they will just laugh and they will eventually get what $I$ am saying and teach me how to say it correctly and then they'll try to speak my language. Where as if you go in and just do your work and you don't at least make an effort than they aren't going to make an effort either and it is going to be miserable and it is going to be a very long 12 hours.

In reviewing the transcripts, listening to the taped interviews, coding and tabulating all the feelings, there were also emotions experienced by the nurses that caused them to feel inadequate, helpless, frustrated, fearful, pressured by time constraints, resentful and angered.

\section{Feelings of Inadequacy}

Feelings of inadequacy and impotence, which led to confusion about how to proceed with care, were those 
mentioned by each participant. The common cause for the feelings of inadequacy hinged mainly on the inability to share a common language. A nurse practitioner told how she doubted her technical skills when she tried to explain to a Mexican woman that she was pregnant:

Well I said maybe I am wrong you know but still in the back of my mind, I know this lady is pregnant. So I am mixed up because tumors are a little different. The lady wasn't sick. I would think somebody that had a tumor that size would be ill. She wasn't ill-appearing at all. She looked fairly normal. So anyway I lay her down and all that and I am still sticking with my diagnosis. I said, "I think you are pregnant". And she said, "that is impossible". "Have you ever been sexually active". "No I am a virgin" (the patient). Fine. okay. Well I am getting a little bit nervous in a way just wondering well maybe I am wrong. The first thing to do is rule out a pregnancy, bottom Iine. We didn't have any prenatal type of equipment. So I used my regular stethoscope and I swear I can hear a heartbeat.

The following experience occurred on a pediatric hematology unit when this nurse participant cared for a seriously ill Mexican child. No one in the family spoke English:

I remember right away feeling a little bit insecure and not competent or not able to care for the family because I didn't speak the language. I remember right away getting one of the other nurses that could interpret for me and in a way I felt kinda small and I felt like gosh that is not fair I should be able to do this. You know I felt like I was giving up part of my job to her in that I should of been the one explaining the procedure because they were going to go and do surgery. I felt like it (the patient) was mine and I felt like I should of been the one to do that stuff. My first impression was that I need to learn. I felt sort of like I was letting them down or like I wasn't as good of a nurse as I could of been because I could of known this language and then $I$ 
could of cared for them better. We couldn't communicate well enough through just sign language and trying because I could speak a few words in Spanish and that she (the nurse) would come and help so that I was to call her. They did seem more open and accepting of me and I guess I felt a little more comfortable.

The next participant retold of her experience trying to find ways of helping a Southeast Asian family understand about their child's illness:

Well you just are at a standstill. You can't go further. You are not communicating or the child's not getting better. You are just at a closed door and there is no place to go and you feel blocked. You just keep trying to see if there is an open door somewhere maybe it is to your left and you were looking to your right and you just keep trying different approaches. You feel like you're not being able to educate them so they might be back with similar problems again. You're concern is whether or not the complete message is getting conveyed or whether the translation is even correct (when using a translator).

One participant described how she attempted to obtain information from the family when a common language was not shared.

Whenever I have a patient who is of a different language and whenever the family is there rather than make direct contact with the family, I will always go for the patient. I will always look at the patient and all my hand gestures will be directed toward the patient. That way they know that I am trying to talk to them. I want to get first hand information from them. It is like having your patient sitting in a bed looking at you through a glass and you can't reach your hands through the glass. That is impotence. You can't get beyond the glass to touch that patient and you can't hear that patient and that patient can't hear you. There is just a big window between you, a barrier that you can't get passed and he can't get to you and you can't get to him.

In the Labor and Delivery Department, a volunteer described 
how she felt when the only English speaking member of a

family was not present:

I don't feel very secure. You know, you are just wondering how can you know if she is going to have this baby or whatever so one of the ways I think is just making sure that before the husband leaves, tell him to let me know when she feels like pressure. I feel helpless when I can't communicate.

The feeling of a pediatric nurse practitioner with many years of experience, was one of inadequacy. She related her feelings as follows:

So I really kinda was at a lost of how to proceed because I assumed that I was going to scrape on my limited Spanish to communicate with this mother. And I said to her, "what language do you speak?". And she said, "Pacific Island". I did not know what to do next because I have worked with a lot of Spanish speaking patients and you know that is hard enough to communicate but with this lady who didn't speak any English or very little English and no Spanish I really didn't know how to proceed. And I felt frustrated. The way she handled her baby and the way she had her baby dressed and all was appropriate and I just assumed because her skin was darker than mine that she spoke Spanish because that was our two major language groups in this particular clinic setting. I was kind of stunned when I knew I wasn't going to reach her in my marginal language. My feeling was that I wish I was in an office where I didn't have to deal with these kinds of issues. Wouldn't it be nice to be in an all English speaking environment with mothers that understood me and could read? You know because we also had a lot of written material that we gave to moms and we had them in English and Spanish because these were our two prevailing cultures or languages but you know this was impossible. I felt inadequate.

Another recorded her feeling in the following way:

When there is someone of a different culture and they speak your language, you can at least communicate and you can identify the differences and you can work around them or work with them. 
And you can also reach that patient better.

Because you know if you take a certain route that is culturally appropriate you are more likely to develop a better rapport and you might get better compliailce and he can understand what your trying to do for him and vice versa. And when there is a culture gap, an ethnicity gap and a language gap you don't have that opportunity. It makes me feel like I can't do my job as well as I would like to. It feels like more of a gap.

A participant told how she felt when she tried to communicate in the patient's language in which she was not proficient. The setting was an obstetric unit:

Well you muddle through. You can't get your questions through and you can't understand what she is trying to tell you. You end up with egg on your face. I mean we have had one instance where we had this little Hispanic who told us who her doctor was and she kept saying a name to me and I went ahead and called the doctor in the middle of the night and it was not his patient. In going back, we found out it was another doctor! I mean that is a big issue, that is a big issue.

Another view on feeling inadequate:

It's the feeling that there is something you need to do, but you do not know what it is. You feel almost impotent. You don't know how to proceed. It is awkward and it almost makes you a little angry to know that there is something you should do but you don't know what and you have no way of knowing.

One nurse explained how she felt when encountering patients

who were non English speaking:

I think the main thing was that in the beginning, I felt so overwhelmed, scared and frustrated and I had to just tell myself, "just relax you will get through this. Just do the best you can and nobody will do any better". It worked out. Yeah, you know so it was very satisfying so you kinda have to keep telling yourself, you don't really have bad outcomes. Things usually work out. It is just that you get so panicked in the beginning. It worked out and it was a very nice encounter. 
It had a good outcome and it made it alot more rewarding.

Feeling Helpless

The difficulty in communicating was reason for the feeling

of "utter helplessness"!

I guess even though you make an attempt to understand other people's languages basically that there is no way you can be able to reach everybody. You just hope to reach the majority of them and I don't know what to think.

\section{Feeling Frustrated and Angry}

Some volunteers were very honest in their descriptions of feeling frustrated and angry when patients could not speak English. From a Labor and Delivery Room perspective, this nurse told her story:

For years I would ask a patient if she was having any vaginal bleeding and instead $I$ was asking her if she had any wine between her legs. I didn't know that the word I was using was not the proper word for bleeding (sangre) and I was asking, "do you have sangria" and they would look at me like I was crazy. It is frustrating for the nurses. Now I say, "Anyone of you speak English?". If you do, you speak up now or you're going to be in big trouble if you don't. I don't want to hear a word of English after that. This person said to me, "how long do I have to wait before my next pain medication", and I said wow! You know we put a lot of humor in it now and you can laugh at it but the thing I resent is for all these years, patients didn't even try to tell me and you know you try so hard to fumble through the few little Spanish words. I have another problem. I can interview a patient in Spanish but then when they answer me, I have not the faintest idea what they say because I can say a few words, they think I speak Spanish. When you really need the help, they won't give it to you but when it is to their convenience, they speak English, not all, some 
really don't know and that is okay.

When needing to give critical information, one nurse stated:

Well you are really frustrated alot of the times and you feel like you are not giving them informed consent or you can't really tell them how their child is doing that day. You can't tell them anything.

One felt that patients should take some responsibility:

I feel frustrated when they can't communicate with the health care provider. You need to bring someone along with you that can serve in that role (interpreter) because the health care provider, in most cases, can't be expected to know all the

Asian languages for instance. We had a couple who were deaf mute and they usually brought their interpreter. So I feel like people have a responsibility, as a consumer, to make sure they get something out of a situation. That it shouldn't all be on the health care provider when you know their not going to speak your language.

A public health nurse told her story and what frustrated her the most:

One time I opened the refrigerator (her client's home) and it was full of cockroaches. I even said to the mother, "you know there are cockroaches crawling in here?" She said, "Yes, I told the landlord." That would be intolerable to me.

Having to prepare a child for surgery was also frustrating when time was of the essence and others depended on the nurse:

In that particular case, it was probably less of an emotional challenge as it was just a physical challenge of getting the work done that needed to be done in a quick manner. The surgeon wanted to get into surgery right now. He does not want to wait for this and that for consent and an interpreter to come. In a way, you feel very frustrated because their kinda looking at you and I feel it is funny because with doctors, if you're bilingual they have a lot of respect for you and in the hospital it seems that they think your 
nursing skills are just as well even if your not bilingual but they seem to favor the nurses that know how to speak both languages and it makes life alot easier for them. So in a way you kinda feel like, gosh, I could be doing a lot more in this situation. I could be doing better. He would really like it if I were the one instead of having to call somebody else every time (to interpret). I could be right there with them explaining everything.

A participant shared her feelings about "Middle Eastern" women in labor:

I think some patients, and I only say about Middle Easterners, because I see alot of those. They're a bit more difficult to care for in that they are not as stoic. They do alot of yelling and you don't know what to do. If you give them something for the pain then I guess it will help. Oh then that really makes you feel like, gosh, I need somebody in here to stay, because then you have the options they can have, and they don't know what would be best for them and so, many times all of the Middle Eastern people do have extended families and that somebody is able to speak English."

\section{Feeling Worried and Threatened}

One nurse described her feeling of anxiety and being threatened when male members of a family came to her unit:

I think a little worried because of the fact that they were Indians from Pauma Valley and I worked in the emergency room where we have had situations like that and I have seen how they have come in. often, they drink too much.

Another nurse worried about being accepted because of her own particular accent and because she had been told that the gentleman patient did not speak English:

Well I was worried because the referral said that he didn't speak English and I was wondering if I would be accepted because I was not even an Anglo person who spoke perfect English. Well, I do 
speak good English but I have an accent and I was just afraid that he would reject me.

\section{Feeling Pressured by Time Constraints}

Most nurses believed that each of them had only a limited amount of time during a shift, to complete their nursing care tasks, whether in the hospital, public health or ambulatory care settings. This added to their feelings of pressures in dealing with patients when verbal communication was impossible or at best limited:

I encouraged her (the mother) to tell me about it (the problem) and if it was a question about whose theories were best than we could discuss it. It all takes time and time is always of an essence when you are working. I think that maybe plays a part in what pressures you feel as a provider and in any given situation particularly when you are dealing with extra issues. It takes more time.

For obstetrical nurses, the causes of time pressures are somewhat different:

If they don't speak your language or if they don't understand what you say, it is very difficult. If they are ready to deliver and the doctor is not there and you don't want them to push, you have to get everything done and it takes a lot of time. Yes, it is a concern, the language barrier. It is one of the biggest concerns because of things that we may not find out, or things that you want to tell her. Penicillin, she is allergic and has a severe anaphylactic shock, for example. She failed to tell us. Sometimes, she needs ampicillin or she needs penicillin. We give it and then what happens? She might say, "I was allergic to penicillin and they gave it to me anyway" but she never communicated with us about it. And when you lift the sheet and find they have had a c-section and you see the scar. You didn't know.

Trying to assess a patient's symptoms through nonverbal 
communication is another source of time pressures:

Well, I was like, oh my gosh this is going to take forever and then I will still not know what is going on. When I had initially said, "What seems to be the problem?", she was pointing that she hurts from her stomach.

For another nurse, it was the lack of time that created the problem:

We are overworked. We have too many people to see

in too short a time and therefore, we are rushing to get to the next person rather than realizing that this person needs to be taken care of adequately first.

Time was also associated with job gratification and indirect feedback:

Right, there is very little instant gratification. You have to work at it. It is over a long period of time and then sometimes you don't even see the results but you will hear later on, later down the road.

\section{Feeling Resentment}

In drawing her conclusions about Mexican American patients, another nurse told of her feelings as she described an incident that caused her to be resentful:

Well with a lot of them, (Mexican Americans), I feel resentment because I feel they have made no effort to learn to speak the language. They live here. I don't think if we went to another country that they would be so accommodating. We have the majority of our Hispanic people who come in and want to know, "do you speak Spanish?" And then some people go a step more and they demand to have a Spanish speaking nurse, "Well why don't you have a Spanish speaking nurse." Well that's because the burden is on our shoulders to provide it for them. They are not going to make an effort and they have people who come in with them who speak English and many times the Hispanic patient speaks not fluently but they have an understanding. You 
don't know it until after they have delivered and then, God has performed a miracle, and they speak English and you know you resent it.

She added:

That you are struggling and fumbling with the language and speak their language. And all the time they can understand you and all the time you make a fool of yourself. You pronounce it (a word) wrong. You pronounce it different. I called a patient garbage for two hours before she corrected me and all that time she was laughing at me.

\section{Feeling Discriminated Against By Clients}

Although only two nurses felt discriminated against, it seemed crucial to explain their feelings. One nurse was Chinese and another was West Indian. In the words of the Chinese-American nurse:

I do resent the fact that they (patients) study you and test you out. I constantly have to prove myself and I get tired of it after a while. I constantly have to prove myself. I have to be better than other people or I will be gone. You think that, oh boy, well what did I do, you know, because when people group you like into the Oriental race. You know you are oriental then your thinking, boy, you know I try my best what did I do wrong?

The nurse from the West Indies felt that the term melting pot was "a crock". For her, Americans believe that "melting pot" means "learning English" and becoming "Americanized". Sometimes she feels the need to ask the patient, "Do you want a Black nurse?" despite the fact that she's not African American, she is Jamaican. She also stated that she does not like the way Black Americans are treated. In her country, everyone is "treated the same". 
Everyone is equal whether white or Black.

All volunteers were able to express their perceptions and sensations without reticence. The only instances in which permission was sought from the researcher was when they wanted to mention specific geographic locations in which their patients lived.

\section{Summary}

This chapter's goal was to present the findings and the final constituents of the phenomenon of the lived experiences of nurses' interactions with ethnically diverse clients. The major constituents were categorized into two levels: Level I as the perceptions of the experiences and Level II as the feelings of the experiences. The perceptions included participants becoming aware of their clients' ethnicity through a variety of characteristics, beliefs, practices, and presumptive clues. Drawing conclusions about clients' ethnicities were based on nurses past experiences and assumptions. Challenges, such as language and reciprocal understanding were also outlined.

The Level II constituents explored the feelings or emotions that nurses experienced while caring for these same clients. These feelings were labelled and presented in statements made by participants. Some of these emotions included feelings of heightened awareness, empathy, compassion, of being accepted and trusted, feeling a sense 
of triumph, reward, satisfaction and challenge. The experiences also included feelings of inadequacy, frustration, anger, worry, feeling threatened, being pressured by time constraints and feeling resentful of the clients. Two of the nurses described how they experienced discrimination while caring for patients who were not members of their ethnic group. 
Final Constituents of the Phenomenon

Table 3

\begin{tabular}{|c|c|}
\hline Perceptions & Feelings \\
\hline $\begin{array}{l}\text { Becoming Aware of the } \\
\text { client's Ethnicity through: } \\
\text { physical characteristics, } \\
\text { body adornment, dress } \\
\text { language: lack of English } \\
\text { fluency or speaking another } \\
\text { language } \\
\text { presumptive clues: } \\
\text { address, legal status, } \\
\text { surname and intuition }\end{array}$ & $\begin{array}{l}\text { feelings of heightened } \\
\text { awareness, empathy and } \\
\text { compassion } \\
\text { feeling accepted and } \\
\text { trusted in building rapport } \\
\text { feeling a sense of triumph, } \\
\text { rewarded and satisfied } \\
\text { feeling challenged } \\
\text { feeling inadequate }\end{array}$ \\
\hline $\begin{array}{l}\text { Drawing Conclusions about } \\
\text { Client's Ethnicities: } \\
\text { role of past experiences } \\
\text {. }\end{array}$ & $\begin{array}{l}\text { feeling frustrated and } \\
\text { angry } \\
\text { feeling worried and } \\
\text { threatened } \\
\text { feeling pressured by time }\end{array}$ \\
\hline $\begin{array}{l}\text { Making Assumptions about } \\
\text { Clients: } \\
\text { based on patient's personal } \\
\text { attributes }\end{array}$ & $\begin{array}{l}\text { constralnts } \\
\text { feeling resentment } \\
\text { feeling discriminated } \\
\text { against by clients }\end{array}$ \\
\hline $\begin{array}{l}\text { Taking Note of Challenges: } \\
\text { language as a barrier } \\
\text { reciprocal understanding }\end{array}$ & \\
\hline
\end{tabular}




\section{Chapter Five \\ DISCUSSION OF THE FINDINGS}

The goal of this Chapter is to provide a synthetic description of the phenomenon, explore and discuss the findings of this study which include perceptions and feelings experienced by the nurse participants. Both levels of constituents will be discussed and linkages will be made with the review of the literature. As much as is possible, conclusions will be drawn and presented.

\section{Synthetic Description of the Phenomenon}

The fifteen "lived experiences of nurses" interactions with ethnically diverse clients" can be described as a combination of perceptual and emotional moments in which nurses became aware of their client's ethnicities in relation to physical characteristics, body adornments, dress, language, beliefs and practices and other presumptive clues. Conclusions were based on the nurses' past experiences which allowed assumptions to be drawn about certain ethnic groups. Challenges were noted when language presented a barrier in communications and reciprocal understanding was questioned. These experiences were a 
blend of emotional moments which contained feelings of heightened awareness, empathy, compassion, acceptance, trust, triumph, reward, satisfaction and challenge. The experiences were also tempered by feelings of inadequacy, frustration, anger, a sense of time pressures, resentment, and in some instances, discrimination by the clients.

\section{Enlightened Awareness}

Van Kaam's Approach

"... I study (people) as the originator and cultivator of (their) world...". "It is most helpful for me to make my first approach that of a phenomenological method, which does not confine or restrict the phenomenon under study to a structure of established theoretical constraints" (Van Kaam, 1966 pp. 292, 296, 298). The use of a phenomenological approach for this study was appropriate because it provided the participants with a milieu in which they could relate their experiences as they recalled them.

Each human experience is at once full of "complexity and fluidity" represented in "each specific moment of the experience" (Van Kaam, 1966, p. 299). Although each person cannot always explain a personal experience in "psychological statements", one becomes aware of what has been lived through via "primordial human awareness" (Van Kaam, 1966, p. 303). According to Van Kaam, "Awareness cannot be proved" (p. 304) although every human being knows 
what they have experienced. The nurses in this study were no exceptions to this theorem. They were able to vividly recollect experiences in which clients were ethnically different than themselves in a variety of interactions and settings. All fifteen participants were articulate, emotionally engaged and willing to share their experiences. The researcher was able to not only understand their descriptions but to actually see and feel what they lived through. As a member of a particular culture, in the case of this study both American and nursing, the researcher was able to comprehend what the volunteers meant when they described the perceptions and the feelings evoked by their nursing care experiences. Van Kaam (1966) observed that "...other people do understand what is basically meant when somebody says "I am happy", "I am bored", or "I feel sad" (p. 312).

\section{Perceptions}

Perception is defined as the ability to grasp, become aware of or comprehend "objects". This information can be acquired through intuition, the senses and awareness (Neufeldt \& Guralnik, 1991, p. 1002). In this study, the nurses explained the perceptions they experienced in interacting with ethnically diverse clients.

Becoming Aware of the Client's Ethnicity of interest to note in this particular study was the 
fact that nurses themselves decided to which ethnicity their patients claimed membership. The fifteen participants made these decisions based on physical characteristics; body adornment and dress. Language seemed to be the prevailing clue in assuming ethnicity, whether because of a lack of English fluency or the client's speaking in some native tongue. Some patients were categorized by beliefs or practices that were shared with or observed by the nurses. Ethnicity was also determined by what this researcher referred to as "presumptive clues", such as address, assumed legal status, surname or intuition. The creation of these perceptions have been supported in the literature.

Fishbein (1962) and Baron and Byrne (1984) identified some determinants of ethnicity as, physical traits (skin color, hair), group practices and behaviors. Other criteria upon which ethnicity is determined are social and behavioral norms of a group such as name and address. Sarason (1982) wrote that living near a particular group, or media information are also influential in forming a schemata about people, thus the determination by the nurses, that if the client was Mexican, the legal status was questionable. Building images of groups is a result of past experiences or "pictures in our heads" as identified by Lippmann (1922, p. 92). Nurses were matter-of-fact in their conclusions about whether their clients belonged to one group or another. The only dilemma occurred if the actual ethnicity could not be 
deduced from the broader culture, for example, to what Asian group a patient belonged. For Parsons (1975), ethnicity acts as an identification of a group. For each participant, determining a client's group membership became a very personal task.

\section{Drawing Conclusions About Clients}

Some of the study participants drew conclusions about the patients, in the context of ethnic belongingness, as a result of their past experiences with similar clients. Maslow (1970) first coined the term "rubric" and defined the process as a subconscious conclusion about people's specific class membership based on the past experiences of the observant. Human perceptions about others are not always based on actual experiences but also on mental images that are envisioned each time like individuals are encountered (Lippmann, 1922). Although mental images were formed by these nurses, none verbalized that this directly affected care. According to the participants, each client was assessed dependent on nursing care needs and observed barriers that emerged from these interactions. One nurse described all (they) Ethiopians as "tall people", "very dark", "different hair", "look like they are not Africans" and "those should be married". This care environment took place in a labor room and the major barrier was identified as language. This nurse decided that the patient was 
Ethiopian and not African, despite the fact that Ethiopia is part of the African continent. This conclusion was created as a result of the participant's past experiences with other patients who, for her, shared similar attributes. Although this client was placed in a rubric, the nurse stated that this did not interfere with the care she received, at least in the eyes of this particular participant. In other cases, nurses attempted to place patients into categories to capture the essence of the ethnicity and to anticipate needs, such as, translators, specific foods and privacy. The existence of the "kernel of truth" theory was also evident in the nurses tales. The use of the collective pronoun "they" was frequently employed to describe a specific ethnic group. Some clients were viewed in the context of group membership rather than as individuals. Lippmann (1922) explained this process as stereotyping.

\section{Making Assumptions About Clients}

Neufeldt and Guralnik (1991) explained the verb "assume" as the taking for granted of a fact, to support or pretend that something exists according to one's own perception. Drawing conclusions seems to be a deductive process, one in which a number of facts is reduced to define a particular idea. For the nurses in this study, the whole of their past experiences seemed to influence the conclusions they made about a particular client's ethnicity. 
In contrast to this, assumptions about patients appeared to emerge from an inductive process. An example was the experience of one nurse who assumed that a Mexican woman's "humble appearance" identified her as a member of an indigenous group rather than as a professional which she indeed was. In one interaction another client was assumed to be knowledgeable because she "looked like upper echelon and educated" yet had to be convinced that she was pregnant due to misinformation she had received earlier in her education.

\section{Taking Note of Challenges}

One of the challenges most frequently mentioned was the presence of language differences. This created more difficulty for nurses and was also the primary reason for producing feelings of triumph, challenge, inadequacy and frustration as the participants attempted to ascertain some level of reciprocal understanding between themselves and the clients. The use of verbal language in the United States is very significant. Here, communication is considered as "low context" because of its dependence on the spoken word rather than on the nonverbal message (Martin, Wimberly \& O'Keefe, 1994). Difficulties in verbally explaining, assessing and educating presented the greatest challenges for nurses. Each wanted to understand the client's needs and, in turn, be understood. The nursing literature identified language 
differences as the most common concern in nursing since 1923 (Committee for the Study of Nursing Education, 1923). Although this Committee encouraged nurses to learn the language of the clients, the challenge is greater in areas like San Diego County where clients are apt to speak one of ninety languages.

The participants described the need for "miming", "pointing", "touching", "show and tell" in order to create a context in which information was reciprocally transmitted. Nonverbal and body language was considered crucial in identifying pain, depression, isolation and grief among their clients and family members. The latter was found very helpful as a resource if one member could communicate in English or Spanish.

Most nurses related the challenges of understanding patient needs, behaviors, beliefs, practices and the desire to be understood. As a challenge, language was more difficult to master and overcome. Baron and Bryne (1984) acknowledged that information about clients affects the quality of communications and the care experience. In essence, this is what the participants grappled with when faced with language differences in the care dyad. In addition, the nurses did not perceive themselves as adequately prepared to translate nonverbal cues. 
Feelings

Feelings are subject-directed, that is, they are emotions experienced by the subject in response to perceptions about the object source (Van Kaam, 1966). Human experience is a "perceptual-emotional Gestalt" (p. 326). All experiential occasions contain "gestalten" or individual responses made up of elements that form a mosaic of feelings and responses (Neufeldt, 1991)

All fifteen participants in this study experienced both pleasant and unpleasant feelings while engaged in the interactions. There were emotions of heightened awareness, empathy, compassion, acceptance, trust, rapport, reward and satisfaction. The same encounters also stimulated feelings of inadequacy, frustration, anger and time pressure. For two volunteers, there was also the sense of being discriminated against by clients.

Feelings of Heightened Awareness, Compassion and Empathy Nurses described their emotions in gaining a sense of awareness about the patient's ethnicity. Compassion and empathy were described as feeling "sorry" for someone, or the "shoe is on the other foot". The emotions were more frequently experienced when a common language was not shared by both the nurse and the client. In addition to mandated care measures, the nurses' assessments and care plans were sometimes based on what the nurse believed she would need or 
want if she were the patient. Arnold and Boggs (1989) referred to this activity as empathic understanding that occurs when the caregiver identifies some shared and unshared attributes. This develops as both members of the dyadic relationship become familiar with each other. At the time of the interviews, few of the volunteers identified any knowledge or education that assisted them in understanding the process that they were experiencing. Rather, they envisioned their situations as unique, with no set of guidelines. Many of them relied on "intuition" and "experience".

\section{Feeling Accepted and Trusted}

Feeling accepted and trusted was experienced if clients shared personal information with the nurses. One gentleman told a nurse that his wife was living here illegally. She, in turn, outlined the process for him to follow in order to get her status legalized. He followed her advice. Nonverbal cues, such as smiles, symbolized some measure of acceptance and trust on the part of the clients toward the caregiver.

\section{Feeling A Sense of Triumph, Reward and Satisfaction}

During interpersonal communications, both members of the dyad began to experience each other's persona at some point in time (Arnold \& Boggs, 1989). Nurses expressed a 
sense of triumph when they were able to somehow overcome a language difficulty or other barrier and gain awareness about the patient's needs or observed the patient's nonverbal language as a sign of some mutual understanding. These moments stimulated feelings of triumph as expressed by nurses in terms such as, "hallelujah", "feeling great", and "I felt satisfied". One nurse shared that she knew she was taking a risk of becoming infected by not wearing a mask with an active tuberculosis patient. However, she felt a deep sense of satisfaction when she attributed this practice to the fact that the patient began to share information with her. She also considered this as the reason for her patient's compliance with the medication schedule. The patient's compliance with the schedule also became her reward. Another shared that even a smile and a thank you or some acknowledgement of understanding were rewards enough for her.

\section{Feeling Challenged}

Trying to make families understand how much the patient was being cared for by the nurse was a major challenge in the face of uncommon languages. How would she make the person understand? How was she going to get the information across? How would she assess and educate the patients? Despite the patients' lack of English understanding, some nurses continued to speak English, yet changed the tone and 
speed of their speech. They tried to convey messages through paralanguage and miming. Some utilized interpreters or a family member that spoke English. Learning some basic words in the client's language helped some to meet the challenge. Each found unique ways of establishing communications.

\section{Feeling Inadequate}

Feelings of inadequacy were also experienced by many of the participants. These emotions were expressed as feeling "impotent", "incompetent", "inadequate", "not measuring up", "hampered", "stumbling", "muddle through", and "in limbo". These feelings were experienced when nurses could not understand the patient, could not decide what the patient's feelings and needs were. Being unable to speak the patient's language made the nurse feel as though she "had to give up the patient" to someone who could speak Spanish or other language. Although Arnold and Boggs, (1989) declared that the inability to communicate with a common language constricts the interaction, the participants found ways to overcome this roadblock while experiencing feelings of inadequacy.

\section{Feeling Frustrated and Angry}

Peplau (1991) uncovered the link between the emotions of inadequacy and frustration. Nurses were frustrated 
because the lack of understanding was reciprocal. Some believed that patients should assume responsibility for being understood. If they were in America, "they should learn the language". Others were frustrated because they could not make patients understand the diagnosis or treatment even when they both shared a common language. They were frustrated and angry because that patient or family did not have the same regard for treatment modalities. Those who felt frustrated eventually found ways of transforming this energy into a mutually safe environment for the dyad as described by Peplau (1991).

\section{Feeling Worried and Threatened}

Feeling worried and threatened became part of one nurse's experience. These emotions were caused by the male members of a patient's family. This participant stated that the men were belligerent when they came to the hospital unit while she was in charge. She assessed them as being intoxicated and threatened the nursing staff with a "lawyer" and "newspaper reporters". Her conclusion was that these men were Native American, consequently they "drank too much". According to the nurse these men may have thought that the patient (their family member) had been discriminated against by the nurses. These emotions were also exaggerated by "what is going on in society today", namely, violence that is carried out in many workplaces. 
Feeling Pressured By Time Constraints

Nurses who felt pressured acknowledged that caring for patients who could not speak the language posed a major time constraint. They felt, at once, stressed by needing to get the job done and knowing the client needed more of their time. At times, no support was available to help them through these encounters. Neither family members nor translators were available. The participants felt "rushed". One stated that it took her half of her shift ( 4 hours) to determine what her patient needed. Others took time to communicate through lengthy mimes and gyrations. What became evident is that the participants did not identify any formal preparation for dealing with these particular feelings.

\section{Feeling Resentment}

Some participants described feeling resentful when clients did not "bother to learn English", "being tested by the patients" or "when they expect us to speak their language". They viewed the above patient behaviors as the reasons for making their work more time-consuming and complex. Others stated that they resented "not being appreciated by the patients" and "by the physicians".

\section{Feeling Discriminated Against}

Two of the fifteen nurses shared their feelings about 
being discriminated against by some patients. One was

Chinese and told of the patient's stories of an "Oriental

torture" system they experienced at the hands of other Asian nurses:

I felt disgusted and degraded. Yes, I felt like you can't judge a person by what they look like. You should always judge a person, give them a chance and give yourself time you know. Wow; when the nurse walks in, within the first two minutes, you need to bombard her with questions to see how much she knows? Is she capable of taking care of me? I have encountered this several times before and all I say, this is prejudice. I am their nurse and sometimes patients call me "Oriental torture". I don't think they are directing it at me because I don't recall the things in a particular incident. But you know, you automatically group yourself into that group, the oriental culture, because I am Oriental. Whatever she (the nurse) did wrong or right that she (patient) was unhappy. This particular patient was very unhappy with her care and obviously she didn't like two oriental nurses taking care of her consecutively. If I can recall right that she told the nurse, "go home and I don't want any new nurse to come on". (The patient was Caucasian).

When the nurse was asked how she knew when she was

"discriminated" against, she said:

Yeah they don't know if they can trust you, especially at shift change, and she (patient) is wondering is this particular nurse in for eight, ten, twelve hours? All of a sudden this new nurse comes in and she looks a little different than from this particular nurse and they have to kind of retrain themselves to know this person. After doing this so many years I know you kinda know that the patient is studying you. They start asking questions. They feel me out. They want to know if I know my job even if they asked the other nurse (the same question) twenty times. I feel disgusted.

A nurse from the West Indies stated that she is direct in her approach if she senses that patients are reluctant to 
have her as a nurse. She says:

"Do you want a Black nurse?". Sometimes American whites come to me and say "Oh, oh, you're not Black like the other Americans". If the person's been to the West Indies they know what is in the West Indies. They know about the culture and then they would look at you differently then the Black Americans.

She stated that Black Americans are treated differently here and she does not approve. Her approach is:

Just talk with the patient and find out exactly what the problem is, yeah, and you find out that person tells you something that is not even a truth.

She knows when discrimination is happening but she says she doesn't let it stop her.

\section{Role of the Researcher's Observations}

Once the participants decided which clients they wanted to discuss, the researcher observed the ease with which the memories were recalled. The nurses shared their stories with emotions ranging from sadness to humor depending on the client and the situation in which the interaction took place.

The participants willingly and candidly explained both pleasant and unpleasant feelings. These perceptions predominantly emerged from a combination of experiences, information and other criteria known only to the nurses themselves. The researcher found each participant credible and trusted the explanations of their perceptual and emotional moments associated with their lived experiences. 
A final observation lies in the difference with which perceptions and feelings were explained. The participants seemed more able to describe their perceptions and did so with much self assurance. Explanations were given without hesitation. On the other hand, the description of the feelings seemed to demand more probing or restating of the questions. For example, when the nurses were queried about becoming aware of the client's ethnicity, conclusions or challenges, the responses were quickly stated. When the question "how did you feel?" was posed, several participants hesitated. Others asked the researcher "What do you mean?" Some said they did not know how to explain it but then followed up with an explanation such as, "I felt frustrated" or "I felt relieved".

In attempting to analyze the difference between perceptual and emotional responses, the answer may lie with the direct way the feeling questions were asked ie. "how did you feel" or perhaps the question itself was perceived as personal, revealing and too subjective.

\section{Summary}

In this Chapter, the findings of the study were discussed. A synthetic description of the phenomenon was presented. The final constituents of the phenomenon, the perceptions and feelings, were revealed and explored.

Nurses became aware of their clients' ethnicities 
through physical characteristics, beliefs and practices, and through presumptive clues. In the course of events, nurses drew conclusions about the patient's membership in a particular ethnic group based on past experiences. The participants also made assumptions about the clients during some of the encounters. Language barriers, feeling understood and understanding clients' needs were considered the major challenges in caring for people whose ethnicities were not shared by the participants.

A variety of feelings were identified as well as their causes in the interactions between nurses and clients from different ethnic backgrounds. Feelings of heightened awareness, compassion, acceptance, trust, triumph, satisfaction and challenge were identified as well as those feelings of inadequacy, frustration, feeling under pressure by time constraints and reverse discrimination.

The role of the researcher's observation during the interviews was discussed. Finally, the descriptions of the nurses' perceptions were contrasted with descriptions of their feelings in trying to understand the reason perceptual moments seemed to be more easily shared than those emotional ones. 
Chapter Six

STRENGTHS, LIMITATIONS, AND IMPLICATIONS

In Chapter Six, the strengths of the study will be discussed. The limitations of both this study and the use of a phenomenological method will also be addressed. In addition, implications for nursing research, education and practice will be presented in an effort to suggest other areas needing investigation.

\section{Strengths}

In order to maintain credibility for this study, the participants were clearly described through demographic information and summarized in Tables 1 and 2. Individual nurses were chosen because they had all experienced the phenomenon of interacting with ethnically diverse patients. All were able to recall and articulate their experiences. Common perceptions and feelings became evident by categorizing and analyzing the descriptions made by the participants. Dependability was maintained by requesting the review of the phenomenon's constituents by four expert judges. A description of these experts is included in Chapter Three. The dependability issue was also maintained through the truthfulness of each person's account. The 
perceptions and feelings were reported precisely as they were stated by the participants. Each nurse shared her personal feelings and perceptions as lived in each experience, thus creating reality in the data. The researcher's concerted effort to set aside her personal knowledge about the experience also supported and preserved the issue of confirmability in phenomenology which incorporates the subjectivity of each experience (Lincoln \& Guba, 1985). Each participant became an expert in the dimensions of her own experiences. The issues of accuracy and truth were addressed in the accurate transcriptions of each participant's conversation. Categorization of their stated perceptions and feelings were restated verbatim. As the constituents became apparent, common themes were identified. Review by the expert panel established a measure of "intersubjective concurrence" (Van Kaam, 1966, p. 315). The nurses stories were their own truthful accounts of what happened as they experienced these dyadic interactions and preserved the subjectivity of the data (Husserl, 1967).

Rigor was maintained by performing as thorough a data collection as was possible and completing in depth interviews. In addition, the researcher made an honest effort to listen intently to the participants' stories while setting aside her own knowledge of like experiences (Spiegelberg, 1982). 
The researcher attempted to enter and leave each interview with naivetè, always enthralled by the nurse's ability to recall each client. Each participant was listened to as though this was the first time that each perception and feeling had been heard. The researcher made every attempt possible to listen only to the words of the nurses in order to decrease the potential for introducing bias into this study. All transcriptions were professionally transcribed in order to minimize the opportunity for the researcher to add or delete certain phrases that might alter the stories. Perceptions and feelings were described in the volunteers' own words and were stated to support the major constituents that were identified.

In order to ensure some measure of objective recall in discussing their feelings and perceptions, the participants decided on the interview locations where they felt the most comfortable. This also afforded opportunities for the nurses to feel relaxed and enjoy the experience. Some labelled the experience "fun", "therapeutic" and "needed". They shared that they wanted to be helpful.

The final phase of this study was the ability to build awareness of the meaning of this experience as shared by the participants. As Van Kaam (1966) stated, "the uniform cultural label affixed to an experience helps people to understand one another in general outlines when expressing 
certain experiences" (p. 313).

\section{Limitations}

One limitation to phenomenological research is the difficulty in incorporating a large number of participants because of the time consuming process of interviewing, categorizing and data analysis of all the information. In Van Kaam's (1966) study of "Really Feeling Understood ( $p$. 325)", the preliminary data were obtained from written descriptions provided by 150 female high school students. When information is gathered in this manner, time is not as critical but the subjects cannot be observed. If the researcher is to become immersed in the phenomenon, the personal interviews may be more effective and personal. Time Iimitations occur because the process is very time consuming. The retrospective process of recalling experience may be viewed as a limitation as well. However, for this study's fifteen participants, their experiences seemed to be so memorable to the extent that once they began their stories, the information continued to emerge and flow only interrupted by the researcher's questions posed for clarification purposes.

Jourard (1964) explained that the researcher's ability to "inspirit" or "dispirit" study participants may also act as an advantage or a limitation. This is understood to mean that the interviewer must remain willing to provide the 
volunteers with sharing opportunities that are pleasurable and that encourage understanding; one in which the researcher becomes involved in the moments (p. 21). In these fifteen encounters, the researcher attempted to create an atmosphere that seemed to inspirit the nurses by conducting interviews in locations where the nurses felt comfortable and listening with enthusiasm to each participant. Some nurses stated that the experience was "therapeutic" and "necessary". They verbalized their pleasure at being a part of the study and hoped their stories would be helpful in explaining the meaning of their experiences and be of service to the profession.

\section{Iimitations of Phenomenological Approach}

Barnum and Stevens (1991) wrote that one of the Iimitations of phenomenology is the researcher's bias. However, this can be controlled through the process of bracketing as first described by spiegelberg (1982). The question still remains, to what extent can the researcher's knowledge about the phenomenon be controlled? Subjectivity has also been considered as a possible root of bias. This can be controlled by reviewing data and identifying the essences or meaning of the lived experiences of nurses interacting with ethnically diverse patients, in such a way so as to allow the reader to envision the phenomenon through the subjects' reflections (Barnum \& Stevens, 1991). This 
can be achieved by identifying the common perceptions and feelings and supporting them with the volunteers' own words (Van Kaam, 1967).

For this researcher, the complete immersion of self in each interview provided little opportunity to make any assumptions. Being thoroughly involved in the moment with each nurse and listening to each story intently, with the goal of understanding and clarifying meanings, provided less opportunity to make assumptions. Each interview was unique and reflected each nurse's experience with the phenomenon in question. By utilizing the words of each volunteer in describing perceptions and feelings, the researcher protected the honest, unbiased explanation of the volunteers' experiences.

Even though phenomenological research has limitations, in terms of generalizability, nursing can gain insight concerning the world of others by listening to personal stories and actually seeing through their experiences. This was defined by Husserl (1967) as the "taking notice of something" (p. 100). As humans share their lives, external perceptions can be grasped by researchers who then begin to see the meaning of each existence. "Going back to the things themselves" and exploring lived experiences with an assumption-free mind set can help to understand the world through the lives of others (Sinha, 1969, p. 3; Scudder \& Mickunas, 1985). 
Also, an honest attempt to control the researcher's bias was made by utilizing the words of each volunteer in presenting both their perceptions and feelings.

\section{Implications}

Nursing Research

Both quantitative and qualitative research methodologies are essential in generating theory, redirecting practice and enhancing nursing education. Phenomenology, as utilized in this study, is a descriptive approach to theory development by furthering human understanding about the uniqueness of each person's life experiences (Burns \& Grove, 1987). This current study may assist in laying the groundwork to further explore not only nurses perceptions but feelings about caring for patients with whom there is no ethnic commonality. The appropriateness of having utilized a phenomenological method is because it "...involves learning to become conscious of spontaneous perceptions, or in other words, getting in touch with one's sensations and feelings" (Paterson \& Zderad, 1976, p. 85).

For Watson (1985), nursing can benefit from phenomenology because this approach uncovers human experience as lived by those participants in a particular research study. Given the opportunity to relate their stories, nurses were able to look reflexively on their 
experiences and build expertise that is critical in nursing (Meleis, 1991). Not only did the participants recall their experiences but discussed them without apparent reservations including both pleasant and unpleasant moments. Although the study's goal were met, many more questions were generated due to the richness of the data.

In phenomenology, there lies the opportunity to review certain aspects of culture and ethnicity and explore a person's participation in a particular culture. Results from these explanations can provide insight into the very nature of another's culture (Scudder \& Mickunas, 1985). In general terms, most of the participants in this study did not seem to gain insight from their encounters. Quite the contrary, they described how they really perceived and felt about these interactions, either positively or negatively. The fewer the commonalities shared by individuals, the greater the potential for conflict and misunderstanding (Bauwens, 1978; Henderson \& Primeaux, 1981; Miller, 1982; Roy, 1976; Spector, 1985). These words were reflected in this study. There were moments in which nurses could not understand the patient's ethnic belongingness. One participant shared that it took her half a shift to assess her patient's needs because he could not speak English. Leininger (1988) suggested that efforts continue to develop theories that address ethnic and cultural issues. As a result of this research, it also seems that efforts 
are needed to review relational and stereotyping theories to assist nurses in examining their own perspectives. Parse's Theory of Man-Living-Health (1981) and Watson's Theory of Care (1985) are perhaps appropriate frameworks to build upon. Exposing nursing students to these theoretical frameworks would serve several purposes: to enlighten them about theories applicable in their daily practice settings and to provide them with information about the health beliefs and values of their client population. Butrin (1992) implied that her study did not support nurses' needing to have cultural information about various ethnic groups in order to promote mutuality within a dyadic relationship. However, this researcher identified the problematic consequences that result if nurses do indeed possess a limited ethnic information. For the fifteen nurses in this study, ethnic knowledge deficit seemed to stimulate assumptions and conclusions whether based on factual information or personal perceptions and feelings. Little nursing research exists that addresses the dilemma of ethnic stereotyping in the profession even though theories do exist in other disciplines (Butrin, 1992; Fishbein, 1967). Without research identifying the extent of the causes, effects and consequences of stereotyping in nursing, the education of novices confronted with an ongoing number of new immigrants will become inadequate. The lack of attention given to the phenomenon of stereotyping in 
nursing may also point to the profession's denial of its existence, much like other cultures who deny the presence of inherent illnesses or conditions. To deny the existence of a problem absolves one of the responsibilities for finding solutions.

Implementing a phenomenological research approach in examining experiences of both clients and nurses is both worthwhile and meaningful. Learning more about the self and others can enhance mutual collaboration and appreciation (O'Brien, 1981; Watson, 1985). Becoming familiar with or updating instruments from the social scientists, such as the Bogardus Tool (1967) could serve as basic research tools in quantitative nursing research. Hypothesizing about the attributes of ethnically diverse patients that precipitate stereotyping by nursing might yield critical data that impacts education and practice. Measuring social distance perceptions, levels of ethnocentrism and personal ethnic feelings among nurses could also influence the quality of the nurse/patient dyad.

other research challenges emerged from this particular study that identified the need to expose the profession to the interactional theories already developed by some nurse theorists which underscore common themes that develop in the experience of caring. Nurses must become sensitized to patients' ethnicities. In addition, the profession also has an obligation to prepare for, and support its members 
through these experiential encounters.

Questions worthy of investigation might be:

1. What are the lived experiences of nurses caring for specific ethnic groups?

2. How do nurses create their assumptions and conclusions about ethnic groups?

3. How can nurses become aware of their stereotypic views?

4. Is it possible for nurses to bracket their assumptions and provide ethnic-sensitive care?

\section{Nursing Education}

Phenomenology has the potential to create a "triadic dialogue" in education, that forms a relationship between the knower or educator, the seeker of information or student and the exploration of the meaning in experiential events (Scudder \& Mickunas, 1985, p. 33). This form of research can be defined as one dealing with human interactions as perceived and felt by those within the experience itself. Exposing nursing students to culturally and ethnically sensitive issues might be a beginning point. If people learn stereotyping and prejudice during their formative years and as a product of the socialization process, it is conceivable that by the time one begins nursing education, viewpoints have already been established and are rigidly supported by the individual. 
Examining personal ethnic membership and providing opportunities for values clarification might become effective methods for enhancing students' awareness about their own perceptions and feelings. Learning how to keep assumptions in abeyance while in the midst of a caring experience might assist nurses in providing patient centered care. A review of diverse ethnic histories, caring and nurturing behaviors and practices could raise consciousness about those with whom nurses will interact. Providing learning opportunities for students to reach out into their communities and experience caring in a variety of ethnic scenarios could assist in anticipatory guidance about potential challenges before they occur in the health care environments. The challenge is to develop nursing students as knowers not only of the clients but also of their own perceptions and emotions (Benner, 1984).

The profession can also ask itself in a multitude of ways:

1. What is the experience of being a caregiver?

2. What are the feelings evoked in ethnically dissimilar patient/nurse dyads?

3. How can educators promote non-threatening milieus in which students can share their feelings and learn how to become more aware of their own ethnic beliefs and biases?

4. How can the profession better prepare nurses to practice in a multi-ethnic society?

5. How can nurses learn to communicate without language? 
6. Can nurses practice safely regardless of language barriers between themselves and their clients?

Nursing students must be given support, positive reinforcement, caring critique and opportunities to learn if they are expected to develop into expert knowers. The stories of this study's participants identified the process that each adopted in making decisions about the patients based on various criteria known to them (May, 1990). Only two credited "some cultural course" they had attended in nursing that broadened their awareness about various ethnicities. None admitted that nursing education had provided them with a framework to assist in or support their assessments of clients. How different could their ethnic experiences have been if these nurses had been educationally prepared to set aside their assumptions and conclusions?

\section{Nursing Practice}

Fisher (1989) suggested that nurses have the potential for "...changing forever the course of patienthood" (p. 63). She explained that this belief is a potential result of labeling patients in one way or another. Her study involved affixing levels of dangerousness among the criminally, mentally ill. For this researcher, the question remains, to what level were the client/nurse interactions altered by nurses labeling patients as "these should be married", "they are very demanding", "they are needy", "they are illegal", "they are permissive with their children", "they laugh at 
us"? In 1931, Dr. R. Cabot wrote about his reactions to immigrants in this manner "...chances are ten to one that I should look out of my eyes and see, not Abraham Cohen, but a Jew... I do not see this man at all. I merge him in the hazy background of the average Jew" (Bullough \& Bullough, 1982, p. 7). Is nursing continuing this approach to this day?

There is no better way to appreciate others than to explore their roots, beliefs, perceptions and experiences. In addition, this may build understanding and reciprocal awareness (Harwood, 1981; Ray , 1976). By whatever means, nurses must assess themselves as well as their clients to become more holistically valuing of each individual in the context of the experiential world. Culturally and ethnically sensitive care encompasses three basic elements: knowledge about the patient's heritage, a cultural assessment of the client and a plan of care that is inclusive of the patient's perspective and input (Bullough \& Bullough, 1982).

The literature review identified the presence of labeling in nursing practice to the extent that it created a barrier to entry or re-entry into healthcare settings. In other situations, labeling provided a rationale for the delivery of inappropriate patient care. Stereotyping, labeling or rubricizing are normal events for all human beings. These mechanisms are present in the "process of 
becoming who we are, separating the self from the other" (Gilman, 1985, p. 18). However, the consequential effects of negative labeling or stereotyping are bigotry and prejudice which become tenacious and allow for individuality to be submerged into group belongingness. This study's findings were congruent with the information retrieved in the review of the literature. In phenomenology, the rationale for completing a review of the literature is to provide some comparison between the researcher's findings and the findings of others. It is not to provide analytic support for the researcher's assumptions (Burns \& Grove, 1987).

As nurses experience the world, both as observers and participants, they must remain centered on their own persona as well as on those with whom they interact. Each human encounter potentiates an opportunity to share oneself, to learn from another and to change beliefs, attitudes and preconceptions. This is the essence of human experience. 


\section{REFERENCES}

Adorno, T. (1978). Solo status, salience, attention and attributes: Top of the head phenomenon. In L. Berkowitz (Ed.), Advances in Experimental Social Psychology, 10. New York City: Academic Press Allport, G. (1958). The nature of prejudice. Garden City, N.J.: Doubleday Anchor Books.

American Nurses' Association (1980). Nursing: A social policy statement. Kansas City, KS: American Nurses' Association.

Anderson, J. (1991). The phenomenological perspective. In J. Morse, Qualitative nursing research: A contemporary dialogue. Newbury Park, CA: Sage. Arnold, E. \& Boggs, K. (1989). Interpersonal relationships: Professional communication skills for nurses. Philadelphia, PA: W. B. Saunders Co., Harcourt, Brace, Jovanovich, Inc.

Ashmore, R. \& Del Boca, F. (1981). Conceptual approaches to stereotypes and stereotyping. In D. Hamilton (Ed.), Cognitive processes in stereotyping and intergroup behavior. Hillsdale, N.J.: Lawrence Erlbaum Associates. Austin, F. \& Worchel, B. (1979). The social psychology of intergroup relations, New York City: John Wiley 
Barnum, S. (Ed.) (1991). Nursing theory: Analysis, application, evaluation (3rd ed.). Glenview, Ill.: Scott Foresman/ Little, Brown. Baron, R., Byrne, D. (1984). Social psychology: Understanding human interaction (4th ed.). Boston, MA: Allyn Bacon.

Bauwens, E. (1978). The anthropology of health. St. Louis, MO: CV Mosby. Benner, P. (1985, october). Quality of life: A phenomenological perspective in explanation, prediction, and understanding. Advances in Nursing Science 8 (1), 1-14.

Benner, P. (1984). From novice to expert: Excellence and power in clinical nursing practice. Menlo Park, CA: Addison-Wesley.

Bergum, V. (1991). Being a phenomenological researcher. In J. Morse, Qualitative nursing research: A contemporary dialogue, (pp. 55-71). Newbury Park, $\mathrm{CA}$ : Sage.

Bierhoff, H. (1989). Person, perception and attribution. New York City: Springer-Verlag. Bogardus, E. (1967). Measuring social distances. In M. Fishbein (Ed.), Readings in attitude, theory and measurement, (pp.71-90). New York City: John Wiley. 
Bonaparte, B. (1979). Ego-defensiveness, openmindedness and the nurses' attitudes toward culturally different patients. Nursing Research, $\underline{28}(3)$.

Brainard, A. (1985). The evolution of public health nursing. New York City: Garland Publishing. Branch, M. (1978). Models for introducing cultural diversity in nursing curricula, Boulder, $\mathrm{CO}$ : Western Interstate Commission for Higher Education. Brislin, R. \& Cushner, K. \& Cherrie, C. \& Tong, M. (1986). Intercultural interactions: A practical guide. Beverly Hills, CA: Sage Publications.

Buber, M. (1958). I and thou, (R.G. Smith, Trans.). New York City: C. Scribner.

Bullough, N. \& Bullough B. (1982). Healthcare for the other Americans. New York City: Appleton-CenturyCroft.

Burns, N., Grove, S. (1987). The practice of nursing research: Conduct, critique and utilization. Philadelphia: W.B. Saunders.

Butrin, J. (1992, August). Cultural diversity in the nurseclient encounter. Clinical Nursing Research 1 (3), 238-251. 
Byerly, E. (1969 May-June). The nurse researcher as participant observer in a nursing setting. Nursing Research, 18 (3). Cacioppo, J. \& Harkins, S. \& Petty, R. (1981). Attitudes and their cognitive response and their relationship to behavior. In R. Petty, T. Ostrom, T. Brock (Eds). Cognitive responses in persuasion (pp. 344-346). Hillsdale, N.J.: Lawrence Erlbaum Association.

Carter, R. (1991, September/October). Cultural values: A review of empirical research and implications for counseling. Journal of Counseling and Development 70, $164-172$.

Chaska, N. (Ed) (1978). The nursing profession: Views through the mist. New York City: McGraw-Hill. Committee on Education of the National League of Nursing Education (1921). Preliminary report of University Schools of Nursing 1-20.

Cook, S. (1957). Desegregation: A psychological analysis. The American Psychologist, 12. 1-13. Coss, C. (1989). Lillian D. Wald: Progressive activist. New York City: The Feminist Press. 
Danielian, J. (1973). Cultural awareness scale. In D., Lake, B., Miles and B. Earle (Eds), Measuring human behavior: Tools for the assessment of social functioning (pp.53-56). New York City: Teachers College Press.

Davis, A. (1978). The phenomenological approach in nursing research. In N. Chaska, The nursing profession: Views through the mist, (pp. 186-197). New York City: McGraw-Hill.

DeTornyay, R. (1976). Cultural diversity and the nursing curricula. Journal of Nursing Education, 15 (2), $3-4$

Doob, I (1967). The behavior of attitudes. In M. Fishbein (Ed.), Readings in attitude, theory and measurement (pp. 42-50). New York City: John Wiley and Sons.

Durkheim, E. (1978). The rules of sociological method (8th ed.). (S. Solovay and J. Mueller Trans). University of Chicago: The Free Press of Glencoe. Estes, D. (1978). Before the war: The Japanese in San Diego. San Diego, CA: San Diego Historical Society.

Farber, M. (1968). Philosophical essays in memory of Edmund Husserl. New York City: Greenwood Press. 
Farber, M. (1966). The aims of phenomenology: The motives, methods, and impact of Husserl's thought. New York City: Harper Textbooks.

Fishbein, M. \& Raven, B. (1973). Attitude beliefs scales. In D., Lake, B., Miles, R., Earl (Eds.), Measuring human behavior: Tools for the assessment of social functioning (pp. 18-20). New York City: Teachers College Press.

Fishbein, M. (1967). Readings in attitude theory and measurement. New York City: John Wiley and Sons. Fiske, S. \& Taylor, S. (1983). Social cognition. Reading, MA: Addison-Wesley.

Forman, G. \& Sigel, I. (1979). Cognitive development: A life-span review. Monterey, CA: Brooks/Cole. French, J. \& Schwartz, D. (1990). Terminal care at home in two cultures. In P. Brink, Transcultural nursing: A book of readings (2nd Ed.), (pp. 247-255). Prospect Heights, IL: Waveland Press Frost, S. (1962). Basic teachings of the great philosophers. New York City: Anchor Brooks: Gajendra, V. \& Bagley, C. (1988). Cross-cultural studies of personality, attitudes and cognition. New York City: St. Martin's Press. 
Gallop, R. \& Wynn, F. (1988, February). Escaping borderline stereotypes: Working through the maze of staffpatient interactions, Journal of Psychosocial Nursing and Mental Health Services 26, (2), 16-20, $38-40$.

Ganong, I. (1987). Integrative review of nursing research. Research in Nursing and Health, 10, 1-11.

Gardner, R. \& Taylor, D. (1969). Ethnic stereotypes:

Meaningfulness in ethnic group labels. Canadian Journal of Behavioral Science, 1, 182-192.

George, J. (Ed) (1985). Nursing theories: The base for professional nursing practice. Englewood Cliffs, N.J.: Prentice-Hall.

Gilbert, G. (1951). Stereotype, persistence and change among college students. Journal of Abnormal Psychology, (46), (pp. 245-254). Gilman, S. \& Justice, J. \& Saephern, K. \& Charles, G., (1992/September). Use of traditional and modern health services by Laotian refugees. The Western Journal of Medicine, 157 (3), 310-315.

Gilman, S. (1985). Difference and pathology: Stereotypes of sexuality, race and madness. Ithaca, New York: Cornell University Press. Glazer, N. \& Moynihan, D. (1975). Ethnicity: Theory and experience. Cambridge, MA: Harvard University Press. 
Goffman, E. (1963). Stigma: Notes on the management of spoiled identity. New York City: Touchstone Book.

Goldstein, H. (1981). Social learning and change: A cognitive approach to human services. Columbus, S.C.: University of South Carolina.

Gordon, R. (1975). Toward a general theory of racial and ethnic group relations. In N. Glazer and D. Moynihan (Eds.), Ethnicity: Theory and experience. Cambridge, MA: Harvard University Press.

Grant, A. (1942). Nursing: A community health service. Philadelphia: W.B. Saunders.

Gudykunst, W., (Ed.) (1983). Intercultural communication theory: Current perspectives. Beverly Hills, CA: Sage Publications.

Guralnik, D. \& Friend, J. (Eds.) (1962). Webster's New World Dictionary College Edition, (p. 1430). Cleveland, OH: World Publishing Co.

Gwen, W. (1973). Cultural awareness scales. In D., Lake, M., Miles, R. Earls (Eds.), Measuring human behavior: Tools for the assessment of social functioning, (pp. 53-56).

Hamilton, D. (1981). Cognitive processes in stereotyping and intergroup behavior, Hillsdale, N.J.: Lawrence Erlbaum. 
Hart, J. \& Maraldo, J. (1976) . The piety of thinking.

Bloomington, IN: Indiana University Press.

Harwood, A. (Ed.) (1981). Ethnicity and medical care,

Cambridge, MA: Harvard University Press.

Heidegger, M. (1962). Being and time. (J. Macquarrie and E.

Robinson, Trans.). New York City: Harper and

Row.

Helmreich, w. (1982). The things they say behind your back.

Garden City, N.J.: Doubleday.

Helman, C. (1992). Culture, health and illness:. An

introduction for health professionals (2nd ed.).

Oxford, England: Butterworth-Heinemann Ltd.

Henderson, B. \& Primeaux, M. (Eds.) (1981). Transcultural

healthcare, Menlo Park, CA: Addison-Wesley.

Hewes, D. \& Planalyp, S. (1982). Social cognition and

stereotyping. In M. Roloff, C. Berger (Eds.),

Social cognition and communication. (pp. 104-

147). N.J.: Lawrence Erlbaum.

Husserl, E. (1977). Phenomenological psychology. The

Hague: Martinus Nijhoff.

Hutchinson, J. (1975). Paths of faith (2nd ed.). New York

City: McGraw Hill.

Isaacs, H. (1975). Basic group identity: The idols of the tribe. In M. Glazer, D. Moynihan, Ethnicity:

Theory and experience. Cambridge, MA: Harvard

University Press. 
Jourard, S. (1964). The transparent self. New York City:

Van Nostrand Reinhold.

Kalisch, P. \& Kalisch, B. (1986). The advance of American

nursing (2nd ed.). Boston, MA: Little, Brown.

Katz, D. \& Braly, K. (1967). Verbal stereotypes and racial

prejudice. In M. Fishbein (Ed.), Readings in

attitude, theory and measurement (pp. 32-38). New

York City: John Wiley and Sons.

Katz, D. \& Braly, K. (1933). Racial stereotypes of 100

college students. Journal of Abnormal and Social

Psychology, (28), 280-290.

Kavanagh, K. \& Kennedy, P. (1992). Promoting cultural

diversity: Strategies for health care

professionals. Newbury Park, CA: Sage.

Kearney, A. (1989). Worldview, Novato, CA: Chandler and

Sharp.

Kleinman, A. (1988). The illness narratives: Suffering,

healing and the human condition. New York City:

Basic Books.

Kramer, B. J. (1992, September). Health and aging of Urban

American Indians. The Western Journal of

Medicine, 157 (3), 281-285.

Kuhn, T. (1970). The structure of scientific revolution

(2nd ed.). Chicago, IL: University of Chicago

Press. 
Lake, B. \& Miles, B. \& Earl, R. (Eds.) (1973). Measuring human behavior: Tools for the assessment of social functioning. New York City: Teachers College Press.

Lawless, R. (1979). The concept of culture. Boulder, Co: Westview Press.

Leininger, M. (1988). Leininger's theory of nursing: Cultural care diversity and universality. Nursing Science Quarterly, 1 (4), 152-164.

Leininger, M. (Ed) (1985). Qualitative research methods in nursing. New York City: Green and Stratton. Leininger, M. (1978). Transcultural nursing: Concepts, theories, and practices. New York City: John Wiley.

Leininger, M. (1977). Culture and transcultural nursing: Meaning and significance for nurses. In National League for Nursing (Ed.), Cultural dimensions in the baccalaureate nursing program (p. 89). New York City: National League for Nursing. Lett, J. (1987) . The human enterprise: A critical introduction to anthropological theory, Boulder, CO: Westview Press.

Levi-Strauss, E. (1984). The view from afar. (J. Neugroschel and P. Hoss, Trans.). New York City: Basic Books. 
Lightfoot-Klein, H., Shaw, E. (1990). Special needs of ritually circumcised women patients. JOGNN, 20 (2), 102-107.

Lincoln Y. \& Guba E. (1985). Naturalistic Inquiry. Beverly Hills, CA: Sage Publication. Lindgren, H. (1984). Stereotyping. In R. Corsini (Ed.), Encyclopedia of Psychology, 3, (p. 367). New York City: Wiley Interscience. Lippmann, W. (1922). Public opinion. New York City: Harcourt, Brace. Lipson, J., Omidian, P. (1992, September). Health issues of Afghan refugees in California. The Western Journal of Medicine, 157, (3), 271-275.

Los Angeles Immunization Demonstration Project (1991, September). Immunizations among low income Hispanics in South Central Los Angeles: A qualitative study, (pp. 1-29). Los Angeles, CA: Los Angeles Public Health Service.

Lowenstein, A., Glanville, C. (1991, March). Transcultural concepts applied to nursing administration. J.O.N.A. $21(3), 13-14$. MacGregor, F. (1990). Uncooperative patients: Some cultural interpretations. In P. Brink, Transcultural Nursing: A book of readings (2nd ed.), (pp. 36-43). Prospect Heights, IL: Waveland Press. 
Martin, K. \& Wimberly, D. \& O'Keefe, K. (1994).

Resolving conflict in a multicultural nursing

department. Nursing Management 25 (1), (pp. 49-

51).

Maslow, A. (1970) Motivation and personality (2nd ed.).

New York City: Harper and Row.

May, C. (1990, March). Research on nurse-patient

relationships: Problems of theory, problems of

practice. Journal of Advanced Nursing, 15 (3), 307-315.

Mehrabian, A. (1971) . Silent messages. Belmont, CA:

Wadsworth.

Meleis, A. (1991). Theoretical nursing: Development and

progress (2nd ed.). Philadelphia: J.B.

Lippincott.

Melson, M. (1983). The thoughts of Martin Buber and

Gabriel Marcel: Implications for existential

encounters in nursing. In $\mathrm{H}$. Werley, Annual

Review of Nursing Research, (pp. 211-226). New

York City: Springer.

Merleau-Ponty (1964). The primacy of perception. (A.

Dallery, Trans.). Evanston, IL: Northwestern

University Press.

Miller, A. (1982). In the eye of the beholder:

Contemporary issues in stereotyping. New York

City: Praeger Scientific. 
Morse, J. (Ed.) (1991). Qualitative nursing research: A contemporary dialogue (2nd ed.). Newbury Park, CA: Sage. Morse, J. (Ed.) (1973). Funk and Wagnall's New Encyclopedia, (p. 363). New York City: Funk and Wagnall

Neufeldt, V. (Ed.). (1991). Webster's New World Dictionary, Cleveland, OH: World Publishing Company. O'Brien, E. (1981). Transcultural nursing research: Alien in an alien land. Image XIII, (2), 37-39. Oiler, C. (1982). The phenomenological approach in nursing research. Nursing Research, 31 (3), 178-181.

Omery, A. (1983, January). Phenomenology: A method for nursing research. Advances in Nursing Science, 1 , 49-62.

Orque, M. \& Bloch, B. \& Monrroy, L. (1983). Ethnic nursing care: A multicultural approach. St. Louis, MO: C.V. Mosby.

Parse, R. \& Coyne, A. \& Smith, M. (1985). Nursing research: Qualitative methods. Bowie, MD: Prentice-Hall.

Parse, R. (1981). Man-living-health: A theory of nursing. New York City: Wiley and Sons. 
Parsons, T. (1975). Some theoretical consideration on the nature and trends of change and ethnicity. In $\mathrm{N}$. Glazer \& D. Moynihan (Eds.), Ethnicity: Theory and experience. Cambridge, MA: Harvard University Press.

Paterson, J. \& Zderad, L. (1976). Humanistic nursing. New York City: John Wiley and Sons.

Peplau, H. (1991). Interpersonal relations in nursing: A conceptual frame of reference for psychodynamic nursing. New York City: Springer Publication. Pettigrew, J. (1981). Extending the stereotype concept. In D. Hamilton (Ed.), Cognitive processes in stereotyping and intergroup behavior, (pp. 303329). Hillsdale, N.J.: Lawrence Erlbaum.

Petty, R. \& Ostrom, T. \& Brock, T. (Eds.) (1981). Cognitive responses in persuasion. Hillsdale, N.J.: Lawrence Erlbaum.

Phares, E. (1984). Social learning theories. In R. Corsini (Ed.), Encyclopedia of Psychology, 3. (pp. 339341). New York City: Wiley Interscience. Polanyi, M. (1962). Personal knowledge. London, England: Routledge and Kegan Paul. Polit, D., Hungler, B. (1993). Essentials of Nursing

Research: Methods, appraisal and utilization (3rd ed.). Philadelphia, PA: J.B. Lippicott. 
Primeaux, M. \& Henderson, G. (1981). Transcultural

healthcare. Menlo Park, CA: Addison Wesley. Ragucci, A. (1972, Nov.-Dec.). The ethnographic approach in nursing research. Nursing Research, 21, 485-490.

Reduced immigration favored (1993, July). The San Diego Union Tribune. (p. A-7).

Ricoeur, P. (1976). Husserl: An analysis of his

phenomenology. Evanston, IL: Northwestern

University Press.

Riepe, D. (Ed) (1973). Phenomenology and natural existence:

Essays in honor of Marvin Farber. Albany, N.Y.:

State University of New York Press.

Rokeach, M. (1960). The open and closed mind. New York

City: Basic Books.

Rosenberg, M. \& Hovland, C. \& McGuire, W. \& Abelson \& Brehm,

J. (1960). Attitude organization and change: An

analysis of consistency among attitude components.

New Haven, CT: Yale University Press.

Roy, C. (1976). Introduction to nursing: An adaptation

model. Englewood Cliffs, N.J.: Prentice Hall.

Sahakian, W. (1968). History of philosophy: From the

earliest times to the present. New York City:

Harper Perennial.

Sarason, I. (1972) - Personality: An objective approach

(2nd ed.) New York City: John Wiley and Sons. 
Schutz, A. (1967). The phenomenology of the social world.

(G. Walsh and F. Lehnert, Trans.). Evanston, IL:

Northwestern University Press.

Schutz, A. (1962). Collected papers: The problem of social

reality. The Hague, Netherlands: Martinus

Nijhoff.

Schwartz, B. \& Lacey, H. (1982). Behaviorism, science and

human nature. New York City: W.W. Norton.

Scudder, J. \& Mickunas, A. (1985). Meaning, dialogue and

enculturation: Phenomenological philosophy of

education. Washington, D.C.: Center for Advanced

Research in Phenomenology and University Press of

America.

Silva, M. \& Rothbart, D. (1984, January). An analysis of changing trends in philosophies of science on nursing theory development. Advances in Nursing Science, $1-12$.

Simpson, S., (1989). Nursing and culture at the end of the twentieth century. In M. Ereilich (Ed.). The relevance of culture, (pp. 187-195). New York City: John Wiley.

Sinha, D. (1969). Studies in phenomenology. The Hague, Netherlands: Martinus Nijhoff.

Smith, M. (1982). Persuasion and human action: A review and critique of social influence theories.

Belmont, CA: Wadsworth. 
Snyder, M. (1978). Behavioral confirmation in social interaction: From social perception to social reality. Journal of Experimental Social Psychology, 14, 148-162. Spector, R. (1991). Cultural diversity in health and illness (3rd ed.), New York City: AppletonCentury-Crofts. Spector, R. (1985). Cultural diversity in health and illness (2nd ed.). New York City: AppletonCentury-Crofts.

Spiegelberg, H. (1982). The phenomenological movement. The Hague, Netherlands: Martinus Nijhoff. Stephan W. \& Rosenfield, D. (1982). Racial and ethnic stereotypes. In A. Miller (Ed.), In the eye of the beholder: Contemporary issues in stereotyping, (pp. 92-129). New York City: Praeger Scientific.

Taylor, S. \& Bogdan, R. (1984). Introduction to qualitative research methods: The search for meanings (2nd ed.). New York City: John Wiley and Sons. Taylor, S. (1981). A categorization approach to stereotyping. In D. Hamilton (Ed.), Cognitive processes in stereotyping and intergroup behavior, (pp. 83-113). Hillsdale, N.J.: Lawrence Erlbaum. 
Taylor, S. \& Fiske, S. (1978). Solo status, salience, attention, and attribution: Top of the head phenomenon. In L. Berkowitz (Ed.), Advances in Experimental Social Psychology, 10, New York City: Academic Press.

Tedeschi, J. (1984). Interpersonal perception. In R.

Corsini, R. Ozaki (Eds.), Encyclopedia of

Psychology, 2, 245-148.

Thiederman, S. (1986, August). Ethnocentrism: A barrier to effective healthcare. Nurse Practitioner: American Journal of Primary Health Care, 11 (8), $52-59$.

Tinkle, M. \& Beaton, J. (1983). Toward a new view of science: Implications for nursing research. Advances in Nursing Science, 5 (2), 27-36.

Travelbee, J. (1971). Interpersonal aspects of nursing. Philadelphia, PA: F. A. Davis. Triandis, H. \& Triandis, L. (1967). Some studies of social distance. In M. Fishbein (Ed.), Readings in attitude, theory and measurement, (pp. 199-207). New York City: Wiley and Sons. Tripp-Reimer, T. (1984). Reconceptualizing the construct of health: Integrating emic and etic perspectives. Research in Nursing and Health $7,101-109$. 
Turiel, E. (1983). The development of social knowledge:

Morality and convention, Cambridge, England:

Cambridge University Press.

Van Kaam, A. (1966) . Existential foundations of psychology.

Pittsburgh, PA: Duquesne University Press.

Van Manen, M. (1990). Researching lived experience: Human

science for an action sensitive pedagogy. New

York City: State University of New York Press.

Watson, J. (1988). Nursing: Human science and human care:

A theory of nursing, Norwalk, CT: Appleton-

Century-Crofts.

Watson, J. (1985). Reflections on different methodologies

for the future of nursing. In M. Leininger,

Qualitative research methods in nursing., (pp. 343-349).

Watson, J. (1979). Nursing the philosophy and science of

caring. Boston, MA: Little, Brown.

Wegner, D. \& Vallacher, R. (1977). Implicit psychology: An

introduction to social cognition, New York City:

Oxford Press.

Werley, H. \& Eitzpatrick, J. (Eds.) (1985). Annual Review

of Nursing Research, 3 . New York City: Springer.

Weigel, R. \& Wiser, P. (1975). The impact of cooperative

learning. Journal of Social Issues, 31. 
Widaman, K. \& Little, T. (1985). Contextual influences on sociomoral judgement and action. In J. Pryor \& J. Day (Eds.), The development of social cognition, New York City: Springer-Verlag. Wilder, D. (1981). Perceiving persons as a group: Categorization and intergroup relation. In $D$. Hamilton (Ed.), The development of social cognition, New York City: Springer-Verlag. Wiman \& Mlerhenry (1971). Edgar Dale's "Cone of Experience". In A. Mehrabian, Silent Passages. Belmont, CA: Wadsworth.

Wyer, R. \& Srull, T. (Eds.). (1984). Handbook of social cognition. Hillsdale, N.J.: Lawrence Erlbaum. Zaner, R. \& Ihde, D. (1973) . Phenomenology and existentialism. New York City: C.P. Putnam. Zaner, R. (1970). The way of phenomenology: Criticism as a philosophical discipline. New York City: Pegasus. 
APPENDIX B

UNIVERSITY OF SAN DIEGO CONSENT

TO ACT AS A RESEARCH PARTICIPANT

Reproduced with permission of the copyright owner. Further reproduction prohibited without permission. 
Appendix B

\author{
UNIVERSITY OF SAN DIEGO
}

CONSENT TO ACT AS A RESEARCH PARTICIPANT

\begin{abstract}
This study involves the lived experiences of nurses' interactions with clients who are ethnically different than the nurse involved in the caring relationship. It is performed as partial fulfillment of the requirement for Colette York's Doctorate in Nursing Science.

There are no foreseeable risks with this research.

I agree to participate in this research project and I understand the following that:

1. the time required for this interview will be approximately one hour

2. the nature of my participation involves a taped interview which includes my sharing of demographic information about myself in addition to my account of the topic being researched

3. all data are confidential

4. my participation is entirely voluntary and I understand that I may withdraw at any time without jeopardy to my role as student or as employee

5. it may be necessary for me to be contacted an additional time to clarify information discussed during the taped interview

6. If I should have any questions about the research or need to talk to Colette York, I can contact her by calling 619-259-7159 further understand that I will be reimbursed $\$ 20.00$, to be given to me at the conclusion of the taped interview.
\end{abstract}

Location

Signature of Researcher 
APPENDIX C

DEMOGRAPHIC QUESTIONNAIRE 
1. DATE:

2. PARTICIPANT I.D.\#:

3. AGE:

4. ETHNICITY:

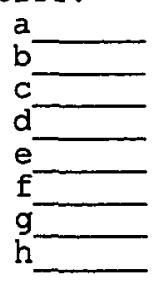

African American

Anglo American (specify ethnic group)

Mexican American

Native American (specify tribe/nation)

Asian American

Pacific Islander

Latin American

Ethnicity with which you identify yourself

(specify)

5. GEOGRAPHIC LOCATION IN WHICH YOU WERE REARED:

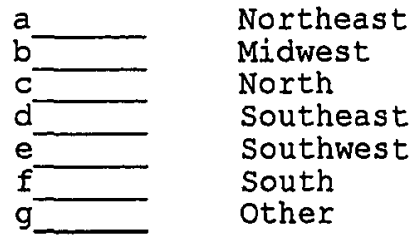

6. IDENTIFY ALL DEGREES YOU HAVE EARNED:

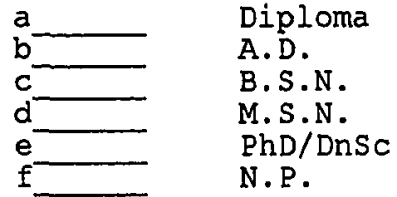

7. ȦREA OF EXPERIENCE OR SPECIALIZATION:

8. YEARS OF NURSING EXPERIENCE:

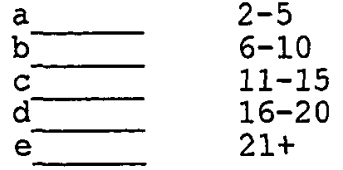

9. TRAVEL OUTSIDE CONTINENTAL U.S. (Specify countries) 
APPENDIX D

INTERVIEW GUIDE

Reproduced with permission of the copyright owner. Further reproduction prohibited without permission. 


\section{INTERVIEW GUIDE}

1. Think about the most memorable patient or patients you interacted with who were not members of your ethnic group.

2. Tell me how you determined that the patient/patients ethnicity was different than yours?

3. Tell me the story:

a. what was the situation in which you interacted?

b. what occurred between the two of you?

c. were their other family members and community involved?

4. How did you feel?

5. What did you do?

6. How did you react or behave?

7. What part of the patient's ethnicity played a role?

8. Describe, if you can, some challenges in this encounter (pleasant, difficult, satisfying, frustrating, educational).

9. What was most memorable about the experience?

10. What have you learned from or brought out of these encounters?

11. How has this or these experiences affected your interactions with other members of this ethnicity or other ethnic groups? 
APPENDIX E

LETTER TO THE PANEL OF EXPERTS 
LETTER TO THE PANEL OF EXPERTS

Appendix E

Dear Dr.

I have chosen you as one of the expert judges to review the constituents of the phenomenon that I am studying, "The lived experiences of nurses' interactions with clients who are ethnically diverse". I have implemented Van Kaam's (1966) model which suggests that the researcher submits the final constituents (categories) that emerge from the data to a panel of judges, for their review, in order to maintain another level of trustworthiness regarding the study. The questions to be asked are:

1. Are these constituents different from those of other interactions?

2. Do these constituents, identified as perceptions and feelings, produce an "enlightened awareness" (Van Kaam, 1966, p. 305) about the phenomenon that is being examined?

As you review the categories, could you please comment on their representation of the experience? I would appreciate your feedback within two weeks if this is possible.

I have also provided a self-addressed and stamped envelope for your convenience. If you wish to contact me by phone, my home number is 259-7159.

Your input and willingness to comment will be most appreciated. Sincerely, 
APPENDIX F

THE PRELIMINARY CONSTITUENTS OF THE PHENOMENON

Reproduced with permission of the copyright owner. Further reproduction prohibited without permission. 
PRELIMINARY CONSTITUENTS OF THE PHENOMENON

LEVEL I: PERCEPTIONS (OBJECT-RELATED)

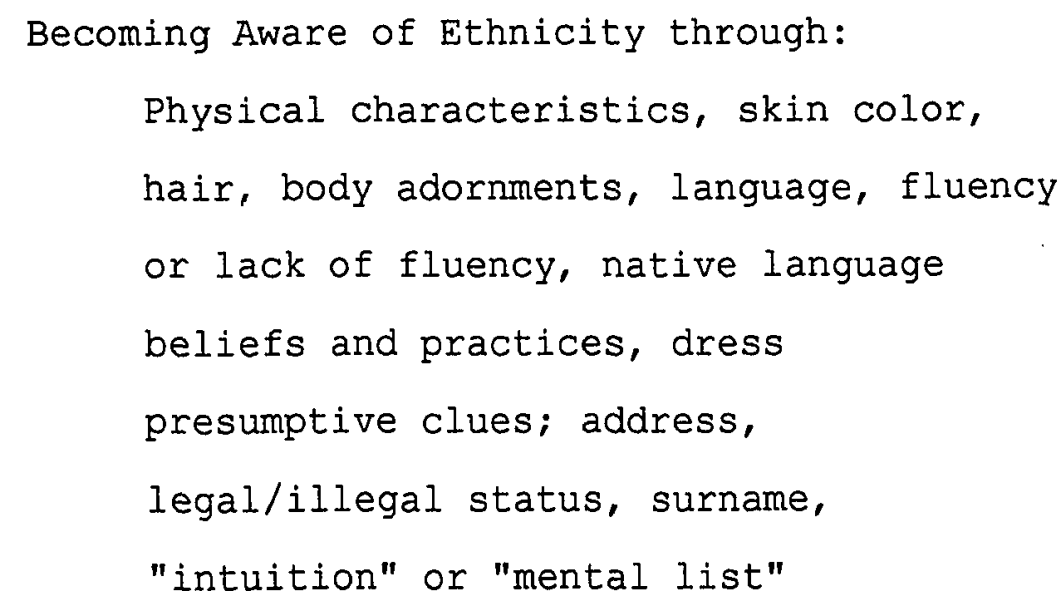

Forming Assumptions/Rubrics about the clients by: comparing self (ingroup) with client (outgroup) drawing conclusions based on past experience, intuition 
Taking note of challenges via:

language barriers

"miming, signing, show and tell"

non verbal cues

need to "reinvent" nursing care measures

(showing caring, use of self as interpreter,

art of touch, using family as resource,

becoming cultural broker, educating through

sign language, "doing the little things"

Discerning the self as exception to:

intolerance

prejudice 
LEVEL II: FEELINGS (SUBJECT-RELATED)

Having positive feelings about the experience:

empathy, challenged, trusted, having

heightened awareness of an ethnicity,

rewarded, proud, satisfied, knowing that

"they will survive in spite of us, tolerant,

accepted, feeling that "you made an impact",

"shoe is on the other foot", preferring one

ethnicity over another

Having negative feelings about the experience:

Needing to "guess", impotent, incompetent,

inadequate, frustrated, anxious, "not

measuring up", afraid, suspicious, "sneaky",

rushed, pressured, "having to give up care to

another", resentful, confused, "hampered", "I

didn't know how to proceed", "worried about

legal aspects", "stumiling through the

language", "wishing I was somewhere else",

"letting patient down", "in limbo", "angry",

feeling "a gap, a glass wall, closed doors",

"very little gratification"

Reproduced with permission of the copyright owner. Further reproduction prohibited without permission. 
Feeling reverse discrimination:

"the patients test you", "they don't

give you a compliment", "I feel I have

to ask if they want a Black nurse", "I

have to be better than someone else" 
APPENDIX G

STATEMENTS THAT IDENTIFY CONSTITUENTS OF THE PHENOMENON 
Appendix G

STATEMENTS THAT IDENTIFY CONSTITUENTS OF THE PHENOMENON

\section{Perceptions}

1. "Dark, dark hair. I knew he was an American Indian... but he also looked Hispanic."

2. "She was blonde and lighter skinned than myself and she spoke with no accent."

3. "They wear long skirts, several skirts of gathered material... and generally they wear their hair braided."

4. "The way she was dressed. It looked like it was bought at Nordstrom's; hair done in a beauty shop, nails done, jewelry...it was not your typical humble laborer that comes over here (from Mexico) to work."

5. "None of them spoke English."

6. "They spoke Spanish and I could tell, they were speaking amongst themselves so I knew before I ever approached them what ethnic group they were in."

7. "He had some burn marks on his abdomen and on his chest."

8. "They (Sikh) live in a commune and the children are raised by a group."

9. "They use the Urgent Care doctor because they can't be bothered making appointments. They don't see the need for continuity in their medical care."

10. "I mean, there was no stress. She had beautiful fingernails."

11. "I knew if she were Hispanic, she would not have spoken English." 


\section{Feelings}

1. "It is rewarding to see people smile and say

'thank you' for trying. They know you are trying."

2. "I went Hallelujah."

3. "The satisfaction for me is that he completed the treatment (for tuberculosis)".

4. "I think coming from a different culture makes me a little bit aware that the general American culture practices differently than we do in Mexico. It makes me a little bit more sensitive."

5. "You have to judge a person individually so if they said all Vietnamese are dirty and they don't know me and think that I am Vietnamese, by all means I am dirty. I am the most pickiest person in sight!" 